\title{
ENGAGEMENT WITH ANGRY FACES DURING ATTENTIONAL BIAS MODIFICATION: INSIGHTS FROM THE N2pc
}

BY

\section{LISA MICHELLE HUNKIN}

\author{
A thesis \\ submitted to the Victoria University of Wellington \\ in fulfilment of the requirements for the degree of \\ Master of Science in \\ Cognitive and Behavioural Neuroscience
}

Victoria University of Wellington

2014 



\begin{abstract}
Healthy individuals show an attentional bias toward threat, and this bias is exaggerated in anxious individuals. Recent studies have shown that training anxious individuals to attend to neutral information can reduce their threat bias and anxiety levels. This training is called attentional bias modification (ABM). However, despite the large literature on $\mathrm{ABM}$, it is still unclear how $\mathrm{ABM}$ achieves its effects. Two mechanisms facilitated engagement with threat, and delayed disengagement from threat - are thought to be involved in the threat bias. In this thesis, I investigated the effects of ABM on engagement with angry faces. First, in Experiment 1 I developed an ABM task to train healthy individuals to attend to either angry or neutral faces. Participants completed a dot-probe task in which they saw two faces - one angry and one neutral - followed by a target that appeared more often in the location of either the angry or neutral face (depending on their respective training condition). Experiment 1 was successful at inducing a bias. Next, Experiment 2 used this task to investigate the effects of $\mathrm{ABM}$ on event-related potentials before, during, and after training. The N2pc component, which provides a measure of attentional engagement, was used to investigate changes in engagement with angry and neutral faces as a function of training. Consistent with previous studies, there was an overall N2pc for the angry face, indicating that participants were engaging their attention with the angry face. However, the $\mathrm{N} 2 \mathrm{pc}$ was not affected by training, even though participants were moving their eyes in the training-congruent direction during training, indicating sensitivity to the training contingency. These results suggest that $\mathrm{ABM}$ does not affect attentional engagement with threat stimuli. Rather, it is likely that an improved ability to disengage attention from threat stimuli underlies ABM's training effects.
\end{abstract}




\section{Acknowledgements}

First, I would like to thank my supervisor, Dr. Gina Grimshaw. I feel like I have come a long way over the last year, in no small part thanks to your gentle guidance and support especially as my thesis due date loomed near! I am so grateful for the time that you have invested in helping me to become a better researcher. It has been a privilege to be your student.

Thank you to the members of the Cognitive and Affective Neuroscience Lab - Angus, Hazel, Jess, Josh, Kealagh, Laura, Lauren, Michael, Sam, Sophie, and Will. I think that we are really lucky to have such a fun and co-operative lab environment, and you guys make it that way. I will miss working with you. Thanks in particular to Josh Foster for training me up in EEG technique and analysis and for being a great sounding board, Angus Chapman for assisting with data collection, and Michael Tooley for saving me when EEG recording went awry.

Thanks also to my family, as I know that I wouldn't be doing what I am today if you hadn't encouraged me to extend myself and give the hard things a go. Thanks Mum for proofreading this thesis and teaching me the difference between 'toward' and 'towards'. And thank you to my partner, Matt, for listening to my practice presentations a few more times than you would've cared to, and for being so patient over the last year.

Finally, I received a Freemasons' Undergraduate Scholarship in Part I of this Master's, and some of that scholarship has supported my study for this year as well. I am very appreciative of the Freemasons' financial support. 


\section{Table of Contents}

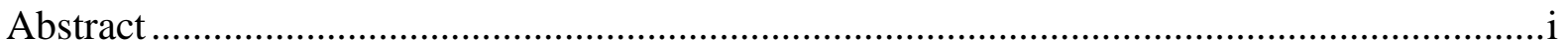

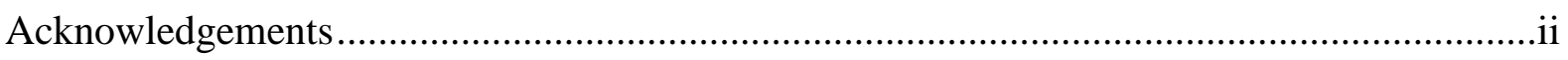

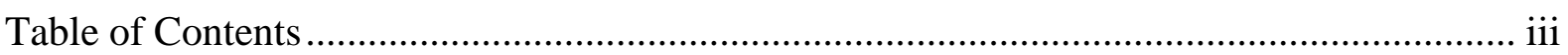

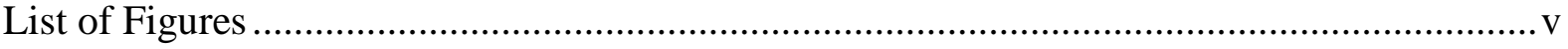

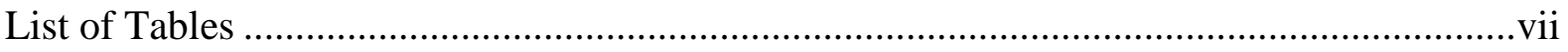

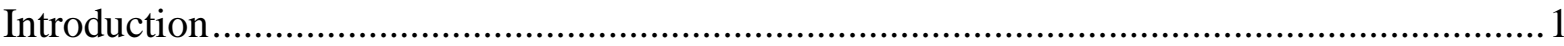

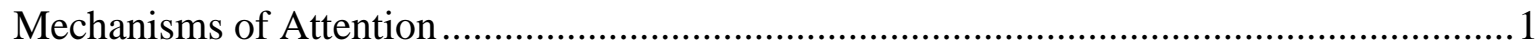

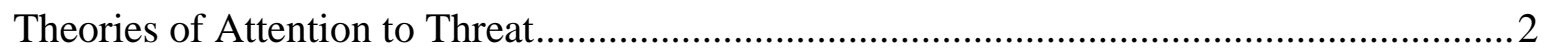

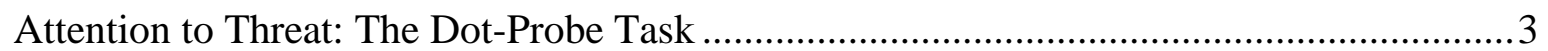

Attention to Threat: Evidence from Other Tasks.............................................................

Attention to Threat: Electrophysiological Evidence ….....................................................5

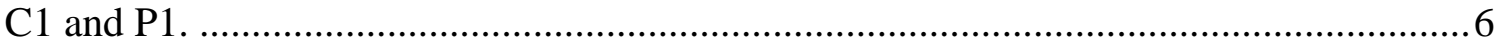

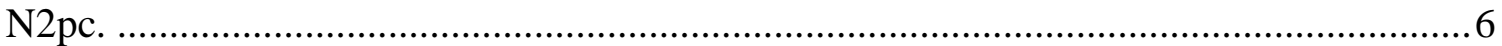

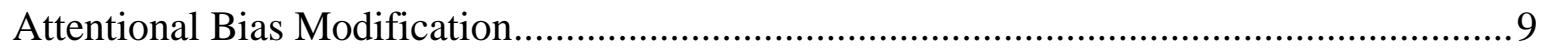

Attentional Bias Modification: Electrophysiological Evidence ........................................ 11

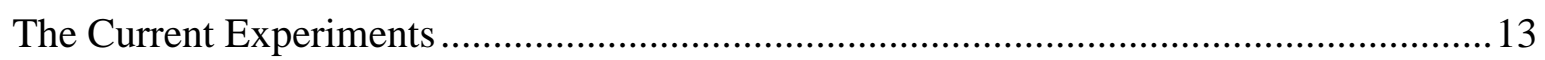

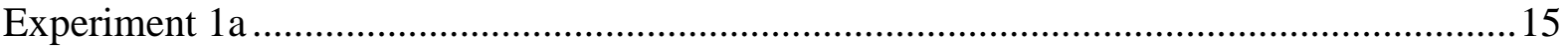

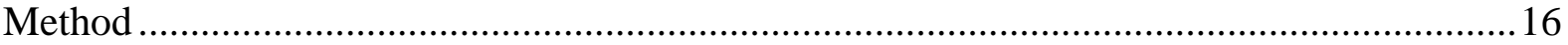

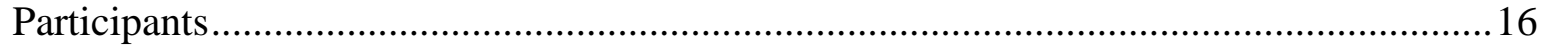

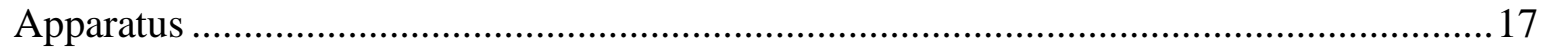

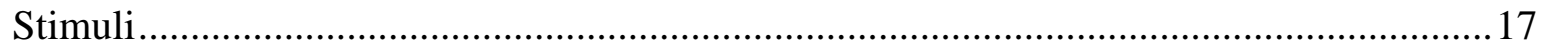

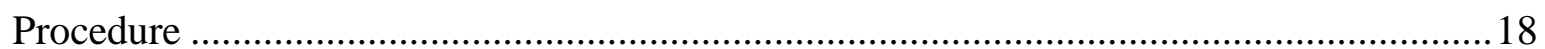

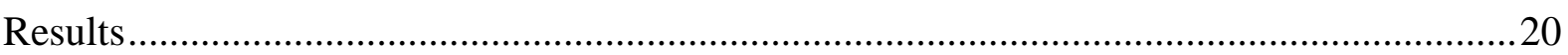

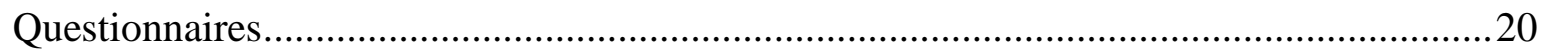

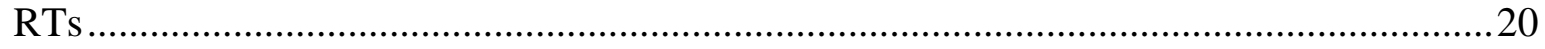

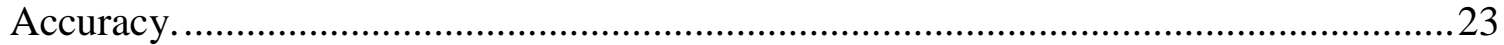

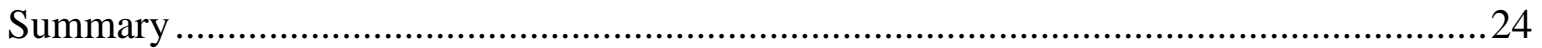

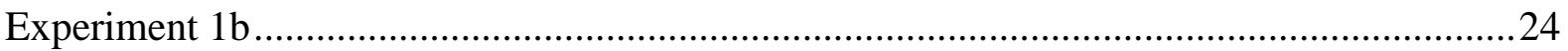

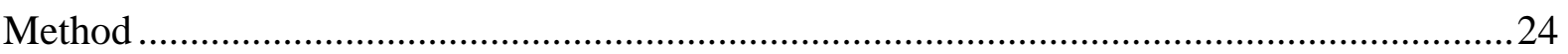

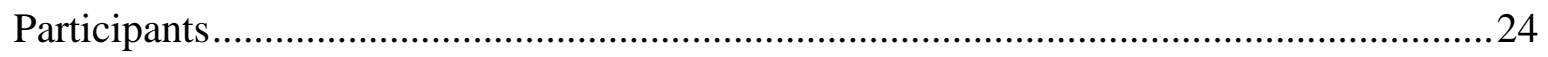

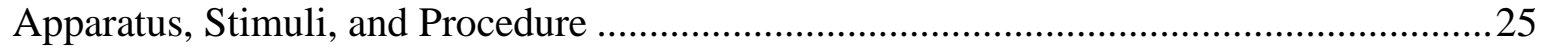

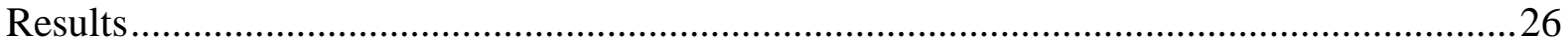

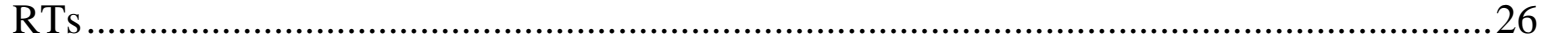




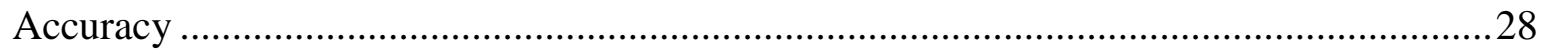

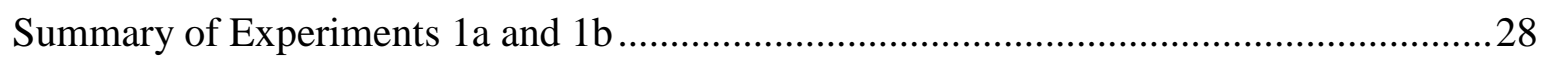

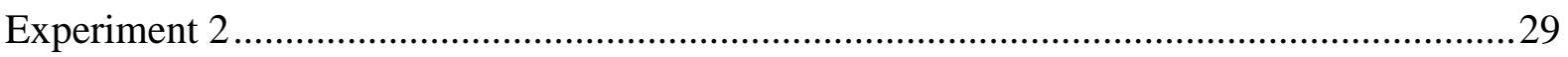

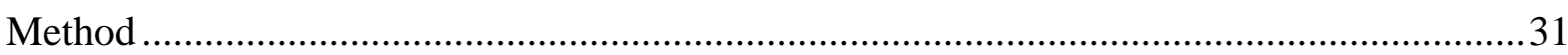

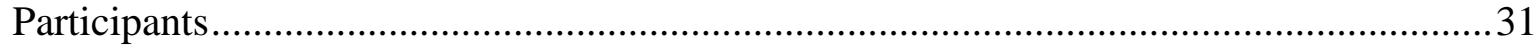

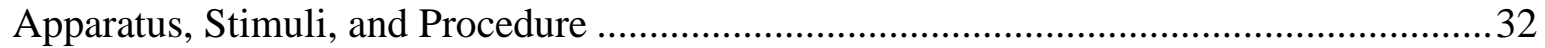

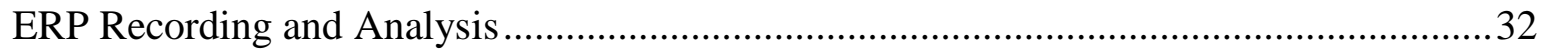

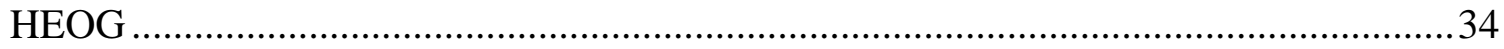

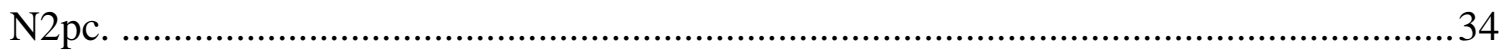

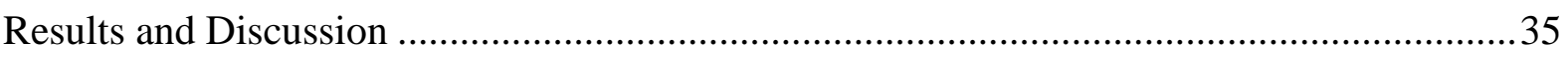

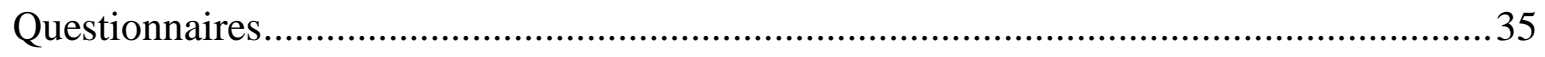

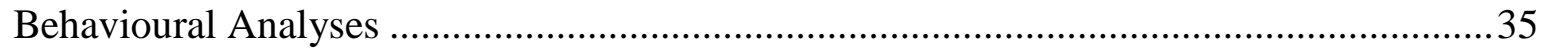

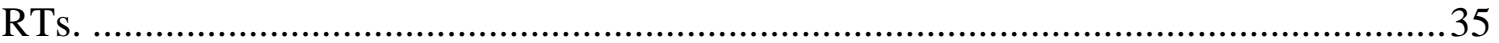

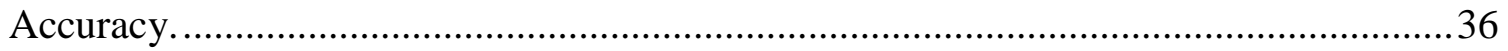

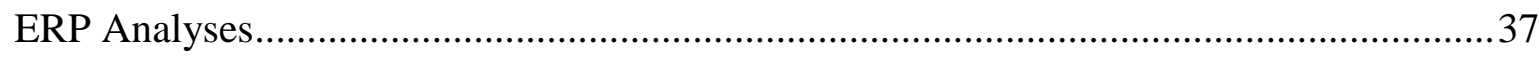

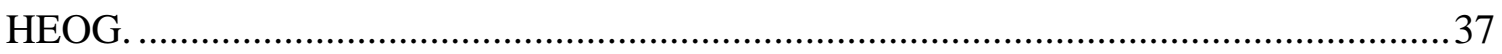

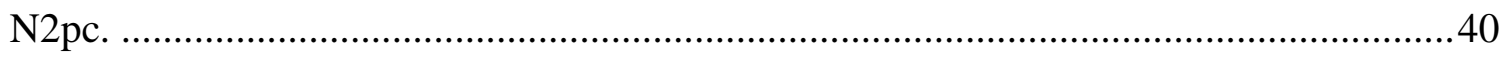

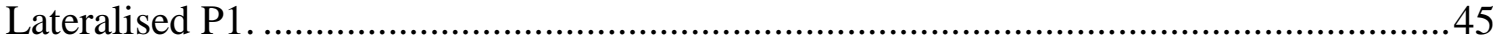

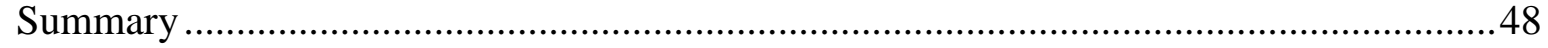

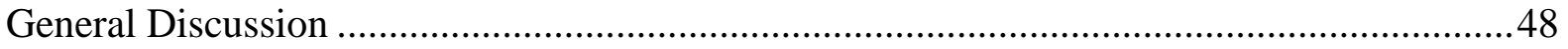

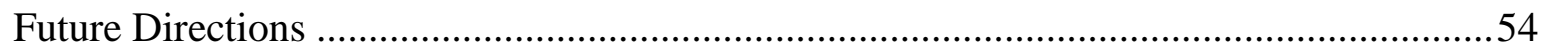

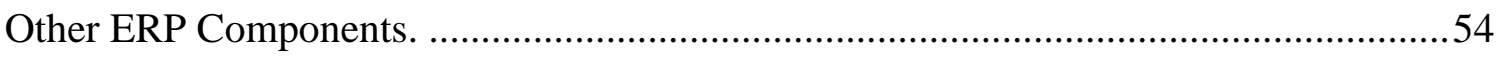

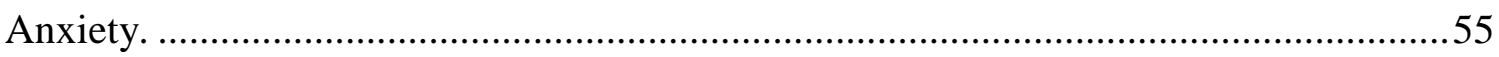

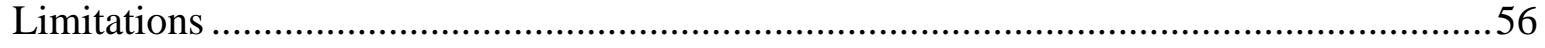

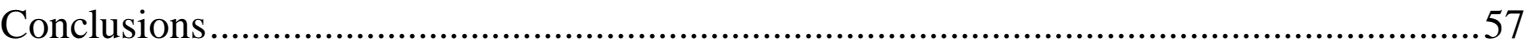

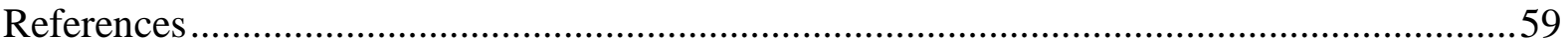




\section{List of Figures}

Figure 1. An example of an N2pc observed in the occipito-parietal electrodes P7 and P8 in response to a bilateral display containing an angry and a neutral face. The amplitude of the electrode contralateral to the angry face is more negative than the amplitude of the electrode ipsilateral to the angry face, indicating engagement with the angry face. Figure adapted from Foster (2013).

Figure 2. Block order and percentage of training-congruent probes in the ABM task in Experiment 1a. 'training-congruent probes' were neutral probes in the neutral training condition and angry probes in the angry training condition. Each unbiased block consisted of 48 trials. Each training and biased blocks consisted of 96 trials.

Figure 3. Example of a face pair presented in the ABM task. .18

Figure 4. Sequence of events in an ABM trial in Experiment 1a. The correct response to the probe display would be 'different'.

Figure 5. Bias scores as a function of block and training condition in Experiment 1a. Positive values indicate a bias toward angry faces. Error bars represent standard error of the mean.

Figure 6. Example of a probe display in Experiment 1b. The correct response to this display would be 'different'.

Figure 7. Bias scores as a function of block and training condition in Experiment 1b. Positive values indicate a bias toward angry faces. Error bars represent standard error of the mean.

Figure 8. Block order and percentage of training-congruent probes in the ABM task in Experiment 2. Each block consisted of 96 trials.

Figure 9. Participant exclusions at each stage of data analysis in Experiment 2.

Figure 10. A: Bias scores as a function of block and training condition in Experiment 2.

Positive values indicate faster responses to the angry probe (indicating an angry bias). Error bars represent standard error of the mean. B: Accuracy bias scores (accuracy to angry probe - accuracy to neutral probe) as a function of block and training condition in Experiment 2. Positive values indicate that participants were more accurate at responding to the angry probe than to the neutral probe (indicating an angry bias). Error bars represent standard error of the mean.

Figure 11. The eye movement index calculated from horizontal EOG in the angry training condition (top) and neutral (bottom) training condition in Experiment 2. A positive score indicates that participants were moving their eyes toward the angry face. 
Figure 12. A: Overall N2pc measured at the P7/P8 electrodes contralateral and ipsilateral to the angry face in Experiment 2. Difference wave is ipsilateral - contralateral activity. B: Scalp distribution of the N2pc difference wave during the N2pc window (180 ms to $320 \mathrm{~ms}$ ).

Figure 13. The eye movement index calculated from horizontal EOG in the angry (top) and neutral (bottom) training condition, calculated from trials and participants remaining after the N2pc filtering and exclusion criteria (see Figure 9) in Experiment 2. A positive score indicates that participants were systematically moving their eyes toward angry faces.

Figure 14. Difference between mean amplitude at the contralateral and ipsilateral (relative to angry face) electrodes during the $\mathrm{N} 2 \mathrm{pc}$ time window (160 ms to $310 \mathrm{~ms}$ ) in Experiment 2. Larger negative values indicate a greater N2pc for the angry face. Error bars represent standard error of the mean. .44

Figure 15. Difference between contralateral and ipsilateral (relative to angry face) P7/P8 electrodes in the $\mathrm{P} 1$ time window $(80 \mathrm{~ms}$ to $120 \mathrm{~ms}$ ) in Experiment 2. More positive values indicate a greater contralateral P1 to the angry face. Error bars represent standard error of the mean.

Figure 16. Contralateral - ipsilateral difference waves calculated from the grand average of each block of P7/P8 electrodes contralateral and ipsilateral to the angry face in Experiment 2 


\section{List of Tables}

Table 1. Mean (SD) of response times as a function of block and probe location in angry and neutral training conditions in Experiment 1a.

Table 2. Mean (SD) accuracy to angry and neutral probes as a function of block and probe location in angry and neutral training conditions in Experiment 1a.

Table 3. Mean (SD) of response times as a function of block and probe location in angry and neutral training conditions in Experiment $1 \mathrm{~b}$ .26

Table 4. Mean (SD) accuracy to angry and neutral probes as a function of block and probe location in angry and neutral training conditions in Experiment $1 b$.

Table 5. Mean (SD) of RTs (ms) as a function of block and probe location in angry and neutral training conditions in Experiment 2

Table 6. Mean (SD) accuracy to angry and neutral probes as a function of block and probe location in angry and neutral training conditions in Experiment 2.

Table 7. Mean (SD) of the late eye movement index ( $\mu \mathrm{V}$; quantified from $350 \mathrm{~ms}$ to $500 \mathrm{~ms}$ ) as a function of block and training condition. Positive values indicate systematic eye movements toward the angry face in Experiment 2.

Table 8. Mean (SD) of the amplitude $(\mu \mathrm{V})$ at P7/P8 electrodes contralateral and ipsilateral to the angry face during the N2pc window (180 ms to $320 \mathrm{~ms}$ ) as a function of block and training condition in Experiment 2.

Table 9. Mean (SD) of the amplitude $(\mu \mathrm{V})$ at P7/P8 electrodes contralateral and ipsilateral to the angry face during the $\mathrm{P} 1$ window $(80 \mathrm{~ms}$ to $120 \mathrm{~ms}$ ) as a function of block and training condition in Experiment 2. 



\section{Engagement with Angry Faces during Attentional Bias Modification: Insights from the N2pc}

Paying attention to threatening information is important for our survival. We have evolved to preferentially attend to threatening over neutral information in our environment (Mallan, Lipp, \& Cochrane, 2013). While our threat bias is often beneficial, it can also be maladaptive; anxious individuals show increased attention to threat compared to healthy individuals, and their increased threat bias is thought to play a causal role in the development and maintenance of their anxiety (Bar-Haim, Lamy, Pergamin, Bakermans-Kranenburg, \& van IJzendoorn, 2007). In support of this hypothesis, training anxious individuals to preferentially attend to neutral information over threatening information reduces their anxiety (see Bar-Haim, 2010; Beard, Sawyer, \& Hofmann, 2012; Hakamata et al., 2010, for reviews). Such training is referred to as attentional bias modification (ABM).

Despite a large body of research that has used ABM to study anxiety, we still do not understand how ABM modifies the threat bias. According to our understanding of the attentional mechanisms that produce the threat bias, there are two stages of cognitive processing on which ABM may be acting; ABM may affect how quickly or easily threat is detected, or it may train participants to control their attention and direct it away from threat.

In this thesis, I investigate the effects of $\mathrm{ABM}$ on attentional processes in non-anxious individuals, in whom these processes are presumably unimpaired. After establishing that my $\mathrm{ABM}$ paradigm can induce a bias toward either angry or neutral faces (depending on the training condition), I use event-related potentials (ERPs) to investigate the brain's response to threatening faces before, during, and after ABM. Changes in ERPs as a result of training can inform which processes are involved in the modification of the threat bias in ABM.

\section{Mechanisms of Attention}

Our visual system has a limited capacity, so we must selectively attend to salient stimuli in our environment at the expense of other, less-salient stimuli. How and why do some stimuli receive more processing than others? The biased-competition model (Desimone \& Duncan, 1995) proposes that all visual information in our environment competes for neural representation. A more salient stimulus will receive greater neural representation, leading to the engagement of attention. This engagement entails enhanced processing at that spatial location. Disengagement must then occur before attention is engaged elsewhere.

There are several different terms used in the literature to describe engagement and disengagement. The terms 'capture,' most commonly used in visual search literature (e.g., Belopolsky, Zwaan, Theeuwes, \& Kramer, 2007), and 'facilitated attention' (Cisler \& Koster, 
2010), are synonymous with engagement. 'Dwell-time' (e.g., Elaine Fox, Russo, Bowles, \& Dutton, 2001) and 'holding' (Koster, Crombez, Van Damme, Verschuere, \& De Houwer, 2005) of attention are synonymous with delayed disengagement. For consistency when discussing these mechanisms, I will use the terms engagement and disengagement in line with their definitions above.

Although the biased-competition model is now almost two decades old, it is still an influential theory of selective attention and its assumptions are supported by recent neural evidence (see Beck \& Kastner, 2009, for a review). The biased-competition model has also informed a number of theories of attention to threat.

\section{Theories of Attention to Threat}

Our understanding of the mechanisms involved in visual attention raises some interesting questions about attention to threat. To what extent is the threat bias a result of facilitated engagement with threat? Or difficulty disengaging from threat? Why do anxious individuals show a greater threat bias than their non-anxious counterparts, and what mechanisms are involved? The past two decades have seen the development of a number of theories of attention to threat to answer these questions.

Common to all of these theories is the premise that threat stimuli are more salient to our attentional system than neutral stimuli, and that threat stimuli are even more salient to anxious individuals than to non-anxious individuals. However, these theories tend to differ in their emphasis on engagement and disengagement mechanisms. On the engagement end of the spectrum, Öhman and Mineka (2001) propose that we have evolved a 'fear module' that is activated by threat. This fear module is sensitised in anxious individuals, causing them to have facilitated engagement with threat. On the other end of the spectrum, attentional control theory (Eysenck, Derakshan, Santos, \& Calvo, 2007; Eysenck \& Derakshan, 2011) proposes that anxious individuals have poor attentional control, causing them to have difficulty disengaging their attention from threat.

Toward the middle of the spectrum are several more integrative theories of attention to threat (Bishop, 2007; Mathews \& Mackintosh, 1998; Ouimet, Gawronski, \& Dozois, 2009). Recent integrative theories suggest that both engagement and disengagement mechanisms are involved in the threat bias in both healthy and anxious individuals. Cisler and Koster (2010) and Bar-Haim and colleagues (2007) suggest that the threat bias arises through both facilitated engagement and delayed disengagement, and that both of these mechanisms are affected in anxiety. These integrative theories are based on a wide range of 
research on attention to threat in both healthy and anxious individuals (see also Cisler, Bacon, \& Williams, 2007, for a review).

\section{Attention to Threat: The Dot-Probe Task}

Behavioural studies provide some insight into the cognitive mechanisms that underlie the threat bias. One of the first tasks used to investigate the threat bias was the modified dotprobe task, developed by Mathews, MacLeod, and Tata (1986). Participants typically see a threatening stimulus (called a threat cue) on one side of the screen and a neutral cue on the other. After the offset of the cues, a probe with no emotional value appears on one side of the screen, and, depending on the task, participants respond with a key press indicating either the location of the probe (detection judgement) or the type of probe (e.g., does the probe contain one dot or two dots; discrimination judgement). Participants will be faster to respond to the probe if it appears in the location where they are currently attending. Thus, faster responses to probes appearing in the location of a threat cue (called threat probes) than to neutral probes indicate a bias toward threat.

In their seminal study, Mathews and colleagues (1986) presented anxious and nonanxious individuals with a modified dot-probe task using threat words (e.g., cancer, failure) and neutral words as cues for a duration of $500 \mathrm{~ms}$. Anxious participants were faster to respond to threat probes than to neutral probes, while non-anxious participants were marginally faster to respond to neutral probes than to threat probes. This finding suggested that anxious individuals preferentially attend to threat words compared to non-anxious individuals.

Mathews and colleagues' (1986) finding has since been replicated in several different anxious populations and with several different types of stimuli (see Bar-Haim et al., 2007; Frewen, Dozois, Joanisse, \& Neufeld, 2008, for reviews). These stimuli include angry faces (Mogg, Philippot, \& Bradley, 2004), threatening scenes (Koster, Verschuere, Crombez, \& Van Damme, 2005; Mogg, Bradley, Miles, \& Dixon, 2004), fear-relevant stimuli (e.g., spiders in spider phobics; Lipp \& Derakshan, 2005), and subliminal stimuli (Mogg \& Bradley, 2002). A subliminal stimulus is one that is presented so that participants are not aware of it (e.g., a very brief presentation, or a masked presentation). These findings suggest that the threat bias in anxiety generalises to several different types of stimuli.

The threat bias can be observed at different stages of processing by varying the amount of time that the cues are displayed on the screen before the probe appears (stimulus onset asynchrony; SOA). Engagement effects should appear when the probe appears soon after presentation of the cues, and disengagement effects should occur later, after engagement 
has occurred. Studies that have compared anxious and non-anxious individuals have found dissociations between the two groups at some SOAs but not at others (but see Mogg, Bradley, de Bono, \& Painter, 1997). At short cue SOAs (less than $100 \mathrm{~ms}$ ), both healthy and anxious participants show a bias toward highly threatening scenes (Koster, Verschuere, Crombez, \& Van Damme, 2005), suggesting that both groups have facilitated engagement with threat within $100 \mathrm{~ms}$. At an SOA of $500 \mathrm{~ms}$, the threat bias often remains in anxious but not nonanxious individuals. At this SOA, anxious individuals show biases toward highly (Mogg, Bradley, Miles, \& Dixon, 2004) or mildly (Koster et al., 2005) threatening scenes, while nonanxious individuals typically show no bias (Cooper \& Langton, 2006, but see Lipp \& Derakshan, 2005). These findings suggest that anxious individuals have both facilitated engagement with and delayed disengagement from threat compared to non-anxious individuals. At longer SOAs (greater than $1000 \mathrm{~ms}$ ), anxious but not non-anxious individuals sometimes show a bias away from threat, indicating avoidance of the threat cue (Koster et al., 2005; Mogg, Bradley, et al., 2004). Threat avoidance may play a role in the maintenance of anxiety (Williams, Watts, MacLeod, \& Mathews, 1988), but will not be discussed further in this thesis.

Although researchers have used SOA manipulations to make inferences about the cognitive mechanisms involved in the threat bias, the bias score itself does not clearly disentangle the contribution of engagement and disengagement mechanisms (Clarke, Macleod, \& Guastella, 2011). The bias score is calculated from the response time (RT) to the probe (Williams et al., 1988). A number of processes have occurred before the participant responds to the probe, including engagement with the cues, disengagement from the cues, and response preparation. Thus, participants will show a bias toward the threat cue if they are still preferentially processing it (facilitated engagement) or if they are slow to shift their attention away from it (delayed disengagement).

\section{Attention to Threat: Evidence from Other Tasks}

Spatial cuing tasks can provide further insight into the involvement of engagement and disengagement mechanisms in the threat bias. The spatial cuing task (Posner, 1980) is very similar to the dot-probe task, except that only one cue is presented at a time, while the other side of the screen remains blank. Faster responses to probes that appear in the same location as the threat cue (called valid threat probes) compared to valid neutral probes indicate facilitated engagement with threat. Likewise, slower responses to probes that appear in the opposite location to the threat cue (called invalid threat probes) compared to invalid neutral probes indicate delayed disengagement from threat. 
A number of studies have used the spatial cuing task to investigate the differences in the mechanisms underlying attention to threat in anxious and non-anxious individuals. Yiend and Mathews (2001) found that anxious participants had delayed disengagement from threatening scenes at a $500 \mathrm{~ms} \mathrm{SOA}$, but found no effects of threat on engagement or disengagement in non-anxious participants. Salemink, van den Hout, and Kindt (2007) found similar results with threatening words. In contrast, Massar, Mol, Kenemans, and Baas (2011) found that both anxious and non-anxious participants had the same level of delayed disengagement from an aversively-conditioned stimulus at a $200 \mathrm{~ms}$ SOA, but that anxious individuals had greater facilitated engagement than their non-anxious counterparts. An aversively-conditioned stimulus is a neutral stimulus that has been presented repeatedly with an aversive event (e.g., a loud noise) and as a result the participant learns to associate the stimulus with threat and acquires a fear response to it. The difference in these findings may be related to the different SOAs, because shorter SOAs are more likely to reflect engagement-related processes, while longer SOAs are more likely to reflect disengagement.

Other behavioural tasks, including the temporal order judgement task (Fecica \& Stolz, 2008; West, Anderson, \& Pratt, 2009; but see Schettino, Loeys, \& Pourtois, 2013), visual search task (Notebaert, Crombez, Van Damme, De Houwer, \& Theeuwes, 2011; Purkis \& Lipp, 2007; but see Tipples, Young, Quinlan, Broks, \& Ellis, 2002; see Frischen, Eastwood, $\&$ Smilek, 2008, for a review), and the attentional blink task (see McHugo, Olatunji, \& Zald, 2013, for a review), have similarly found that non-anxious individuals have both facilitated engagement with, and delayed disengagement from, threat stimuli.

The aforementioned tasks have also been used to investigate differences between anxious and non-anxious individuals. Studies using the visual search task (Eastwood et al., 2005; Rinck, Becker, Kellermann, \& Roth, 2003), and attentional blink task (Cisler, Ries, \& Widner, 2007; Fox, Russo, \& Georgiou, 2005; Trippe, Hewig, Heydel, Hecht, \& Miltner, 2007) have similarly found that anxious individuals have greater facilitated engagement with and delayed disengagement from threat relative to non-anxious individuals. Notably, in all of these tasks, a bias is observed when threatening stimuli compete with other stimuli for attention.

\section{Attention to Threat: Electrophysiological Evidence}

In addition to behavioural responses, neural responses to threat can also provide insight into the cognitive processes that underlie the threat bias. One way to investigate neural responses to threat is to use electroencephalography (EEG). When populations of neurons in the brain communicate in synchrony, the electrical activity that they create can be 
recorded from the scalp using EEG. Event-related potentials (ERPs) can then be derived from the EEG activity. First, the EEG recording is divided into segments that are time-locked to the onset of the stimulus. The stimulus is presented multiple times, and the resulting EEG segments are averaged. With sufficient trials, the averaging process cancels the noise from the EEG signal and only the brain's consistent response to the stimulus - the ERP wave remains. There are many characteristic ERP components that appear in response to particular stimuli or tasks, and the appearance of (or changes in) these components can be used to infer cognitive processes. Differences between ERP components to threat and neutral stimuli can provide insight into how our brains might process these stimuli differently. A key advantage of ERP is that it does not require a behavioural response and has very high temporal resolution, allowing researchers to differentiate between processes that may occur in close succession.

C1 and P1. Two ERP components that have been used to investigate the brain's response to threat are the $\mathrm{C} 1$ and the $\mathrm{P} 1$. The $\mathrm{C} 1$ is the earliest visual ERP component, appearing approximately $65 \mathrm{~ms}$ after stimulus onset, and indexes the first cortical sweep of attention over the environment (Luck, Woodman, \& Vogel, 2000). The P1 is another early component, appearing $100 \mathrm{~ms}$ after stimulus onset, and its magnitude reflects the attentional resources recruited to process the stimulus (Luck et al., 2000). Studies have found that healthy individuals have a greater C1 (Pourtois, Grandjean, Sander, \& Vuilleumier, 2004; but see Santesso, Meuret, \& Hofmann, 2008) and P1 (Santesso et al., 2008) to fearful faces relative to neutral faces. An enhanced P1 to is also observed to subliminally-presented fearful faces (Li, Zinbarg, \& Paller, 2007) and aversively-conditioned stimuli (Stolarova, Keil, \& Moratti, 2006). Taken together, these findings suggest that threat stimuli differentiate from neutral stimuli at a very early stage in visual processing.

Studies comparing anxious and non-anxious individuals have also found differences in the $\mathrm{C} 1$ and $\mathrm{P} 1$ to threat. Anxious or phobic individuals show a greater $\mathrm{C} 1$ to threat faces (Eldar, Yankelevitch, Lamy, \& Bar-Haim, 2010), spiders (Weymar, Keil, \& Hamm, 2013), and subliminally-presented threat words ( $\mathrm{Li}$ et al., 2007), and a greater P1 to threat faces (Rossignol, Philippot, Bissot, Rigoulot, \& Campanella, 2012) than non-anxious individuals. These findings all suggest that anxious individuals have increased early threat processing compared to non-anxious individuals.

N2pc. Another ERP component that can provide an index of preferential processing of threat is the N2pc. It appears in the occipito-parietal region approximately $200 \mathrm{~ms}$ after stimulus onset, and is more negative on the opposite side (contralateral) of the head than the 
same side (ipsilateral) relative to the attended stimulus (Figure 1). Thus, the N2pc can indicate whether a participant is attending to the left- or right-hand side in a lateralised display. Notably, an N2pc is only observed when stimuli are competing for attention (Luck \& Hillyard, 1994). This finding has caused debate about whether the N2pc reflects distractor suppression or target enhancement (e.g., Mazza, Turatto, \& Caramazza, 2009), but despite this disagreement, the literature is consistent in showing that the $\mathrm{N} 2 \mathrm{pc}$ provides a measure of attentional engagement. As a result, the $\mathrm{N} 2 \mathrm{pc}$ has been used widely over the past two decades for this purpose (see Luck, 2011, for a review).

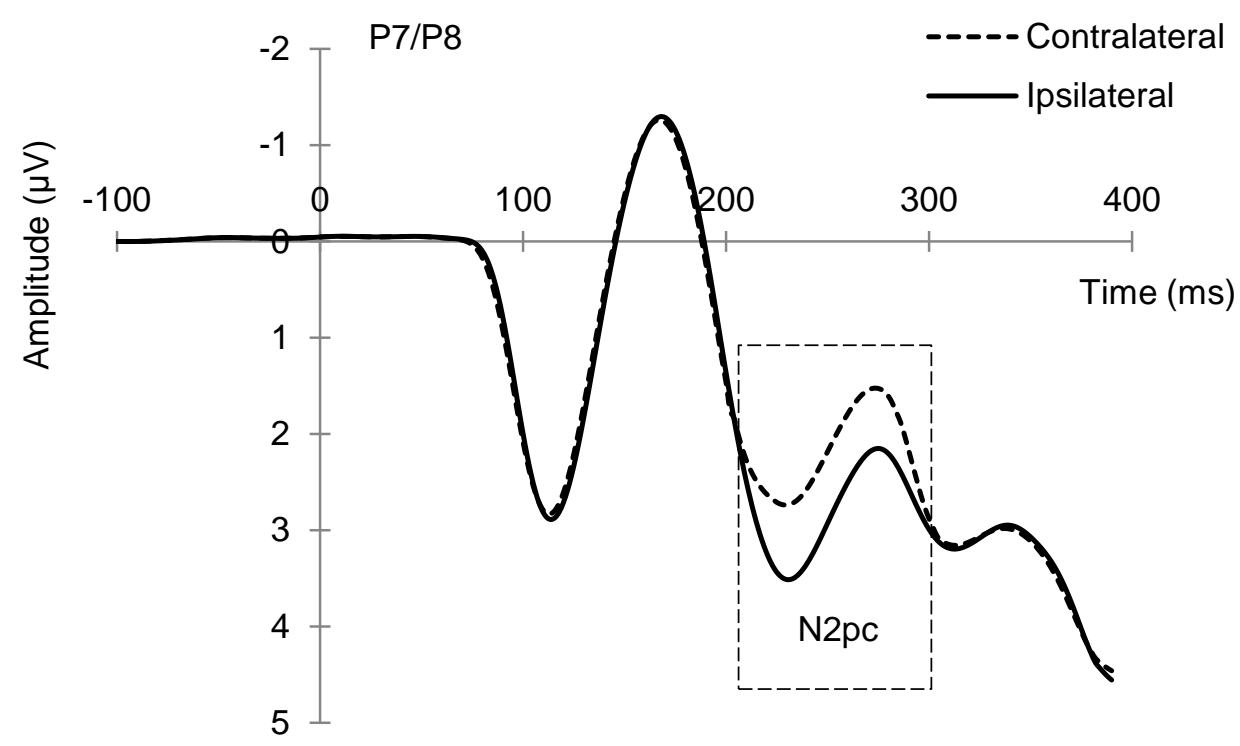

Figure 1. An example of an N2pc observed in the occipito-parietal electrodes P7 and P8 in response to a bilateral display containing an angry and a neutral face. The amplitude of the electrode contralateral to the angry face is more negative than the amplitude of the electrode ipsilateral to the angry face, indicating engagement with the angry face. Figure adapted from Foster (2013).

Because it is a robust component that is clearly affected by attention, the N2pc has recently become popular for studying attention to threat. Studies in both our own laboratory and in other labs have consistently shown that healthy individuals have a greater N2pc for fearful or angry faces, relative to neutral or happy faces in the dot-probe task (Foster, 2013; Grimshaw, Foster, \& Corballis, 2014; Holmes, Bradley, Kragh Nielsen, \& Mogg, 2009; Holmes, Mogg, de Fockert, Nielsen, \& Bradley, in press; but see Brosch, Pourtois, Sander, \& Vuilleumier, 2011) and other tasks (Eimer \& Kiss, 2007; Feldmann-Wüstefeld, SchmidtDaffy, \& Schubö, 2011; Ikeda, Sugiura, \& Hasegawa, 2013; Shaw, Lien, Ruthruff, \& Allen, 2011; Weymar, Löw, Ohman, \& Hamm, 2011; Yao, Ding, Qi, \& Yang, in press; but see 
Lien, Taylor, \& Ruthruff, 2013). These findings suggest that when threat and neutral stimuli compete for attention, non-anxious individuals preferentially engage with threat stimuli.

Notably, this N2pc for threatening faces has also been observed in the absence of any behavioural effects. In several studies, an N2pc for the threatening face (indicating biased engagement with the threatening face) was observed even when there was no difference in RTs to threatening and neutral faces (Eimer \& Kiss, 2007; Foster, 2013; Grimshaw et al., 2014; Ikeda et al., 2013). Why is there dissociation between behaviour and ERPs in the dotprobe task? First, it may be that the behavioural bias measured in the dot-probe task is not sensitive enough and is therefore unreliable (see Cooper \& Langton, 2006, for a critique of the dot-probe task). Second, it may be that because the location of the threat cue does not predict the location of the probe, it is not advantageous for participants to maintain their initial engagement with the threat cue. Participants might engage their attention with threat (as indexed by the N2pc) but then disengage from the threat cue before the probe appears and therefore show no bias when they respond (Everaert, Spruyt, Houwer, \& De Houwer, 2013; Lichtenstein-Vidne, Henik, \& Safadi, 2012). Indeed, in a number of other paradigms that do find a threat bias in healthy participants, they must attend to the threatening stimulus in order to perform the task effectively. It is possible that although healthy individuals show early engagement with threat, they have sufficient attentional control that they can easily disengage their attention from threat when it is not relevant to the task, and as a result, they do not show a measurable behavioural bias (Koster, Leyman, Raedt, \& Crombez, 2006). Thus, the N2pc may be a more direct measure of biased attentional engagement with threat than behavioural measures.

Anxiety also modulates the N2pc for threat stimuli. Anxious individuals show a greater N2pc for angry faces compared to healthy individuals (Elaine Fox, Derakshan, \& Shoker, 2008). This finding again suggests that anxious individuals have increased engagement with threat. The N2pc is also modulated by specific fear; phobics have a greater $\mathrm{N} 2 \mathrm{pc}$ for stimuli related to their phobia than for other threatening stimuli (Buodo, Sarlo, \& Munafò, 2010; Weymar, Gerdes, Löw, Alpers, \& Hamm, 2013).

To summarise, a range of different tasks and methodologies generally suggest that healthy individuals have facilitated engagement with and impaired disengagement from threat, and threat has an even greater effect on these mechanisms in anxious individuals. Although anxious individuals show a greater threat bias than non-anxious individuals, the studies reviewed above have not established a causal relationship between anxiety levels and attention to threat. 


\section{Attentional Bias Modification}

Recent studies have found that modifying an individual's threat bias can affect his or her anxiety levels. In a typical attentional bias modification (ABM) task (Mathews \& MacLeod, 2002), participants are assigned to either a neutral training condition or a control training condition and complete a modified dot-probe task with a $500 \mathrm{~ms}$ SOA. In the neutral training condition, the probe always appears in the location of the neutral cue. It thus becomes advantageous (in terms of task performance) for participants to attend to the neutral cue, since the probe will always appear in that location. In the control training condition, the probe appears equally often in the location of the threat or the neutral cue (for clarity, I will continue to refer to control training consistent with its use here). Participants in the control training condition are exposed to the same cues as participants in the neutral training condition, so any differences between the two training conditions after training are a result of the training contingency. $\mathrm{ABM}$ has provided a unique way to examine the cognitive mechanisms that may be involved in the threat bias and anxiety, since it establishes a causal relationship between change in the threat bias and anxiety levels.

In their seminal study, MacLeod and colleagues (2002) used threat and neutral words as cues in an ABM task. They found that participants in the neutral training condition subsequently had a reduced threat bias and reduced anxiety to a stressor relative to participants in the control training condition. A number of other studies have since replicated these findings in several different anxious populations with words, faces, and other pictorial stimuli as cues (see Bar-Haim, 2010; Beard, 2011; Hakamata et al., 2010; Hallion \& Ruscio, 2011, for reviews).

The ABM literature has typically focused on clinical outcomes in anxious individuals, and as a result few studies have investigated the cognitive mechanisms that might underlie this change in anxiety and the threat bias. Only one study has attempted to directly assess the involvement of engagement and disengagement mechanisms in ABM. Heeren, Lievens, and Philippot (2011) used a variant of the spatial cuing task to train social phobics to either disengage from threat cues or engage with neutral cues. In the disengage condition, participants saw only disgust faces as cues, and the probe appeared opposite the disgust face $95 \%$ of the time. In the engage condition, participants saw only neutral faces as cues, and the probe appeared in the same location as the neutral face $95 \%$ of the time. After training, participants in the disengage condition but not the engage condition had a reduced threat bias and showed reduced anxiety following a stressor. The researchers concluded that disengagement from threat underlies the effects of ABM. However, facilitated engagement 
with threat is often only observed when threat is competing with other stimuli, and there was no such competition in the engagement condition in this study. Thus, these results do not rule out the possibility that engagement is involved in the reduction of anxiety and the threat bias in $\mathrm{ABM}$.

In addition, few studies have investigated the effects of threat training (i.e., training in which the probe always appears in the location of the threat cue) in anxious individuals. Of those that have, results have been mixed. One study found that participants in both the neutral and threat training condition showed reduced anxiety following a stressor relative to participants in the control training condition (Klumpp \& Amir, 2009). This finding suggests that instead of affecting attentional engagement with threat, ABM may instead train participants' attentional control, allowing them to disengage from threat when it is no longer advantageous for task performance. This idea is consistent with attentional control theory, which proposes that the threat bias in anxiety arises from a deficit in attentional control (Eysenck et al., 2007; Eysenck \& Derakshan, 2011). However, studies attempting to replicate since have found both consistent (in non-anxious individuals; O'Toole \& Dennis, 2012) and inconsistent (Heeren, Reese, McNally, \& Philippot, 2012) results. Thus, while there is some evidence to suggest that both threat and neutral training reduce anxiety, presumably by increasing attentional control, more research is needed.

Supporting the theory that ABM trains attentional control, participants' awareness of the cues can also influence the effects of training (Maoz, Abend, Fox, Pine, \& Bar-Haim, 2013). ABM with supraliminal cues reduced anxiety in anxious participants, while training with subliminal cues had no effect. Because conscious awareness of the cues is necessary for ABM's effects, it is unlikely that engagement mechanisms are involved in the reduction of anxiety in ABM.

Because of the clinical emphasis of the ABM literature, very few studies have investigated the effects of ABM in non-anxious individuals. Studying non-anxious individuals may provide some additional insight into the mechanisms underlying attention to threat, because their cognitive mechanisms that produce the threat bias are presumably unimpaired. Of studies of ABM in non-anxious individuals, some have reported no change in the threat bias as a result of training (Eldar \& Bar-Haim, 2010; O’Toole \& Dennis, 2012). Others have found that threat but not neutral training shifts the bias in a training-congruent direction (Browning, Holmes, \& Murphy, 2010; Eldar, Ricon, \& Bar-Haim, 2008). One study has investigated the effect of $\mathrm{ABM}$ on the time course of the threat bias. Koster and colleagues (2010) administered either neutral training or control training to non-selected 
participants. Neutral training reduced the threat bias at late $(1500 \mathrm{~ms})$ but not early $(30 \mathrm{~ms}$ or $100 \mathrm{~ms})$ SOAs. No effects were observed in the control training condition. These findings suggest that neutral training improved disengagement from threat but had no effect on engagement with threat.

Some of the inconsistencies across training studies in non-anxious participants may be related to the way that the threat bias is assessed. A bias score is calculated from the difference in RTs to threat and to neutral probes, so bias cannot be assessed during training because only one type of probe is present. As a result, changes in bias are typically tested by comparing participants' pre-training and post-training bias with a dot-probe task in which the probe appears equally often in the location of the threat or neutral stimulus. Notably, the way that the bias score is calculated means that it cannot be assessed during training. If nonanxious participants have good attentional control (as proposed in attentional control theory; Eysenck et al., 2007), non-anxious participants may acquire a training-congruent bias during training, but this bias might revert back at test when it is no longer advantageous to attend to the training-congruent cue. However, since no studies have investigated attention to threat during training, more research is needed to determine whether and how the threat bias can be modified in non-anxious individuals.

\section{Attentional Bias Modification: Electrophysiological Evidence}

$\mathrm{ABM}$ studies cannot assess the threat bias during training, and post-training assessments produce unreliable results. ERPs may provide more insight into the cognitive mechanisms that are affected by ABM because they are a more sensitive measure of attention and can be measured during training. To date, only three studies have investigated the ERP correlates of ABM.

Eldar and Bar-Haim (2010) assigned anxious and non-anxious participants to either a neutral training condition or a control training condition. Neither anxiety level nor training affected early ERP components (P1 and N1) to the angry cues. However, anxious (but not non-anxious) participants showed changes in later ERP components (reduced P2 and P3, and increased N2) following training. The researchers suggested that these later ERP processes might be related to emotional processing and attentional control. A similar study with nonanxious individuals assigned to either an angry training condition (i.e., in which the probe always appeared in the location of the angry face) or a control training condition found complementary results (Suway et al., 2013). Consistent with Eldar \& Bar-Haim, there was no effect of training on early ERP components (P1 or N1) to the threat cues. Angry training increased the $\mathrm{P} 2$ relative to control training. Taken together, these findings suggest that ABM 
affects late attentional processes (likely related to disengagement) but not early attentional processes (likely related to engagement).

However, another ERP study (O'Toole \& Dennis, 2012) has reported inconsistent findings with the previous two studies. This study used a variation of the ABM paradigm in which participants were presented with angry and happy faces instead of angry and neutral faces in the aforementioned studies. Non-anxious participants were assigned to either a happy training condition (i.e., in which the probe always appeared in the location of the happy face) or an angry training condition. In contrast to the previous two studies, happy training affected early ERP components (P1) relative to the angry training condition. Later components $(\mathrm{P} 2$, $\mathrm{P} 3$, and N2) were affected by training in both training conditions, but there was no difference in these components between the training conditions. These contradictory results may have been due to different task designs. The majority of ABM studies (including those by Eldar \& Bar-Haim, 2010 and Suway et al., 2013) have used SOAs of 500 ms, while O'Toole and Dennis used a SOA of 1000 ms. Longer SOAs may have different effects on the mechanisms underlying ABM.

Unlike the other two studies, O'Toole and Dennis (2012) also presented participants with cue displays with two happy faces (rather than an angry and a happy face). This modification allowed responses to displays containing angry and happy faces (angry displays) and two happy faces (happy displays) to be compared. Happy training decreased the P1 to happy displays relative to angry displays, while there was no effect of angry training. This finding was unexpected, and suggested that happy training reduced the attentional processing of happy faces. While this manipulation improved upon the other two ERP studies in that it directly compared responses to threat and happy cues, the results in O'Toole and Dennis' study are less transferable to the ABM literature because of the different SOAs and task design.

Finally, none of the aforementioned studies assessed ERPs during training; they all compared pre-training to post-training assessments. This issue is particularly relevant to the studies of non-anxious individuals, because they may acquire a training-congruent bias during training but this bias may revert to baseline at the post-training assessment. Thus, changes in ERPs during training could provide more insight into ABM's effect on the threat bias and its underlying cognitive mechanisms.

In summary, the ERP studies of ABM to date have left several areas unaddressed. First, there is some inconsistency between the three studies with respect to the components that are affected by ABM. In addition, none of these studies have investigated ERPs during 
training. Only one study has directly compared ERP responses to threat and non-threat displays. However, this study used a novel ABM task, making its results less transferable to the other ABM literature. In addition, these previous studies have described the effects of $\mathrm{ABM}$ on early and late ERP components, but these components have not been definitively linked to a specific attentional mechanism. That is, the previous studies have indicated whether training affects 'early' or 'late' processes, but not specific attentional mechanisms. The N2pc, which is a robust measure of engagement as described in a large attention literature and growing threat literature, has not yet been studied in ABM. Clearly, there is a need for more studies to investigate the ERP correlates of ABM.

\section{The Current Experiments}

Studies of attention to threat have suggested that healthy people have both facilitated engagement with and delayed disengagement from threat, and that the effect of threat on these mechanisms is even greater in anxiety. Training anxious individuals to attend to neutral information reduces their anxiety, but the extent of the involvement of engagement and disengagement mechanisms in ABM is still unclear. The few studies that have investigated these mechanisms have suggested that disengagement but not engagement is involved in ABM's effect on anxiety and the threat bias. However, there is still some disagreement in the literature, and no studies have examined changes in the threat bias or these mechanisms during training.

The aim of the current experiments was to extend our understanding of the cognitive mechanisms that underlie ABM. Experiments 1a and 1b were behavioural experiments designed to develop an ABM task to train a bias toward either angry or neutral faces in healthy participants. This ABM task was then used in Experiment 2, in which ERPs were used to examine neural responses to threat before, during, and after training. The focus of Experiment 2 was on changes in the N2pc, an ERP component that provides a measure of attentional engagement with threat relative to neutral faces during the dot-probe task and has been used in the literature previously for this purpose (Foster, 2013; Grimshaw et al., 2014; Holmes et al., in press).

All experiments had the same basic structure that was based on the typical ABM training procedure reported in the clinical literature. Participants were randomly assigned to either an angry training condition or a neutral training condition. During a pre-unbiased block, participants completed a dot-probe task in which the probe appeared equally often in the location of the angry and the neutral face (that is, the faces were not predictive of the probe's location). The pre-unbiased block provided a baseline measure of each participant's 
existing threat bias. During the subsequent training block, the probe always appeared in the location of either the angry face (angry training) or the neutral face (neutral training). Finally, participants completed a post-unbiased block in which the faces were again non-predictive, in order to determine whether any effects of ABM persisted beyond training.

In Experiments 1a and 1b, the procedure was altered slightly so that changes in the threat bias during training could be assessed. Instead of a series of training blocks, participants completed alternating training blocks (containing 100\% training-congruent probes; i.e., $100 \%$ angry probes in angry training and 100\% neutral probes in neutral training) and biased blocks (containing $75 \%$ training-congruent probes). The bias was assessed during these biased blocks, which were necessary because the threat bias cannot be assessed during training because RTs to both angry and neutral probes are required to calculate a bias score. Studies in the attentional literature have investigated the effect of predictability on the bias with non-threat cues in a dot-probe task. These studies have found that participants show a training-congruent bias when probes appear in the location of the training-congruent cue $80 \%$ of the time (Lambert, Naikar, McLachlan, \& Aitken, 1999). However, prior studies in the ABM literature have only assessed biases with blocks containing an equal proportion of training-congruent probes and training incongruent probes. Non-anxious individuals may acquire a training-congruent bias during training, but this bias might revert to baseline at test. By including biased blocks in which it was still advantageous to attend to the training-congruent face, I expected that healthy participants would show a training-congruent bias during these biased blocks. In addition, by alternating training and biased blocks, Experiments $1 \mathrm{a}$ and $1 \mathrm{~b}$ established the number of training trials necessary to train a bias, which then informed the number of trials included in Experiment 2.

In Experiment 2, I replicated and extended Experiment $1 \mathrm{~b}$ to investigate the effect of $\mathrm{ABM}$ on neural responses to the faces. Participants completed a pre-unbiased block, followed by training, and then a post-unbiased block. Participants no longer completed biased blocks consisting of $75 \%$ training-congruent trials. The particular ERP component that I was interested in was the $\mathrm{N} 2 \mathrm{pc}$, because it is a measure of attentional engagement. In the preunbiased block, I expected all participants to show an N2pc for the angry face, consistent with previous literature. If $\mathrm{ABM}$ affects attentional engagement with threat, I expected the $\mathrm{N} 2 \mathrm{pc}$ for the angry face to increase during angry training and to decrease during neutral training. Finally, I included a post-unbiased block to investigate whether any changes in the $\mathrm{N} 2 \mathrm{pc}$ persisted beyond training. 
The cue stimuli in the ABM task were pairs of angry and neutral male faces. These faces were selected from the stimuli used by Foster (2013), who found an N2pc for these angry faces in healthy participants. Angry rather than fearful faces were used because fearful faces may be ambiguous about the location of the threat (Williams, Moss, Bradshaw, \& Mattingley, 2005), and may be more relevant to the expresser rather than the viewer (Springer, Rosas, McGetrick, \& Bowers, 2007).

Only non-anxious and non-depressed women were included in the current study. Depressed individuals show a bias at long but not short SOAs, in contrast to the pattern observed in anxious individuals (see Mogg \& Bradley, 2005, for a review). The aim of the current study was to investigate the cognitive mechanisms that are affected by ABM; presumably these mechanisms are unimpaired in non-anxious and non-depressed individuals. To increase the homogeneity of the sample, only women were recruited in all experiments. Women have a higher incidence of depression (Piccinelli \& Wilkinson, 2000) and anxiety (Bekker \& van Mens-Verhulst, 2007) than men, and differ from men in their ability to discriminate threatening faces (Thayer \& Johnsen, 2000). Including only women in the study therefore increased power to detect a training-related change in the $\mathrm{N} 2 \mathrm{pc}$.

\section{Experiment 1a}

The goal of Experiment 1a and 1b was to develop an ABM task that could train a bias in non-anxious participants. This task would then be used in Experiment 2 to investigate ERP responses during $\mathrm{ABM}$. To that end, non-anxious participants were assigned to either an angry training condition or a neutral training condition, and completed an ABM task based on the modified dot-probe task.

Previous studies of ABM have been inconsistent in training a bias in non-anxious individuals. This inconsistency may be caused by the way that bias is tested. The threat bias cannot be assessed during training blocks containing $100 \%$ training-congruent probes (i.e., angry probes in the angry training condition, neutral probes in the neutral training condition), because RTs to both angry and neutral probes are required to calculate a bias. ABM's effect on the bias must therefore be assessed after training, typically with a modified dot-probe task. In this post-training assessment, the probe can appear equally often in the location of the angry or the neutral face. Thus, it is advantageous for the participant to attend to the training-congruent cue during training, but it is not advantageous for them to maintain this bias in the post-training assessment. It is possible that although participants acquire a training-congruent bias during training, this bias disappears in the post-training assessment, 
leading researchers to conclude that $\mathrm{ABM}$ is not effective. To address this issue, in addition to training blocks (100\% training-congruent probes) and unbiased blocks (50\% training-congruent probes), Experiment 1a included biased blocks containing 75\% training-congruent probes. It was therefore advantageous for participants to maintain a training-congruent bias and a bias score could be calculated from these biased blocks.

A secondary goal of Experiment 1a was to establish the number of trials necessary to train a bias. After a pre-unbiased block, training and biased blocks were alternated three times, followed by a post-unbiased block (see Figure 2). The first biased block at which a training-congruent bias was observed would determine the number of training trials in Experiment 2.

Consistent with previous literature, I expected that non-anxious participants would show no bias in the pre- and post-unbiased blocks. However, if the ABM task and the biased blocks were effective in training and testing the threat bias, I expected that participants would show a training-congruent bias during the biased blocks.

\section{Method}

\section{Participants}

Forty three participants were recruited from an undergraduate psychology course at Victoria University of Wellington, and received course credit for their participation. The participants were all women, right handed, had normal or corrected-to-normal vision, and were not currently receiving, and had no history of, treatment for depression. A series of questionnaires to assess participants' mood and experience with depression and anxiety were administered. Mean scores on the Mini Depression and Anxiety Stress Scale (Mini-DASS; Lovibond \& Lovibond, 1995) were $32.0(S D=24.7$, range $=2-94)$, on the Mini Mood and Anxiety Symptom Questionnaire (Mini-MASQ; Clark \& Watson, 1991) were 53.9 (SD= 17.3, range $=35-100$ ), and on the Penn-State Worry Questionnaire (PSWQ; Meyer, Miller, Metzger, \& Borkovec, 1990) were 52.1 ( $S D=15.6$, range $=30-80)$. Participants were excluded from analyses on the basis of neurological disorder $(n=1)$, or if they had total score greater than two SDs from the mean on any questionnaire $(n=2)$, or for low accuracy $(<50 \%$ in any block) in the ABM task ( $n=1)$. The remaining participants (mean age: 18.6 years, $S D$ $=1.50$, range $=17-26$ years $)$ were randomly assigned to either an angry training condition $(n$ $=21)$ or a neutral training condition $(n=18)$. This experiment was conducted with the approval of the Victoria University of Wellington Human Ethics Committee. All participants gave written informed consent before participating in the experiment. 


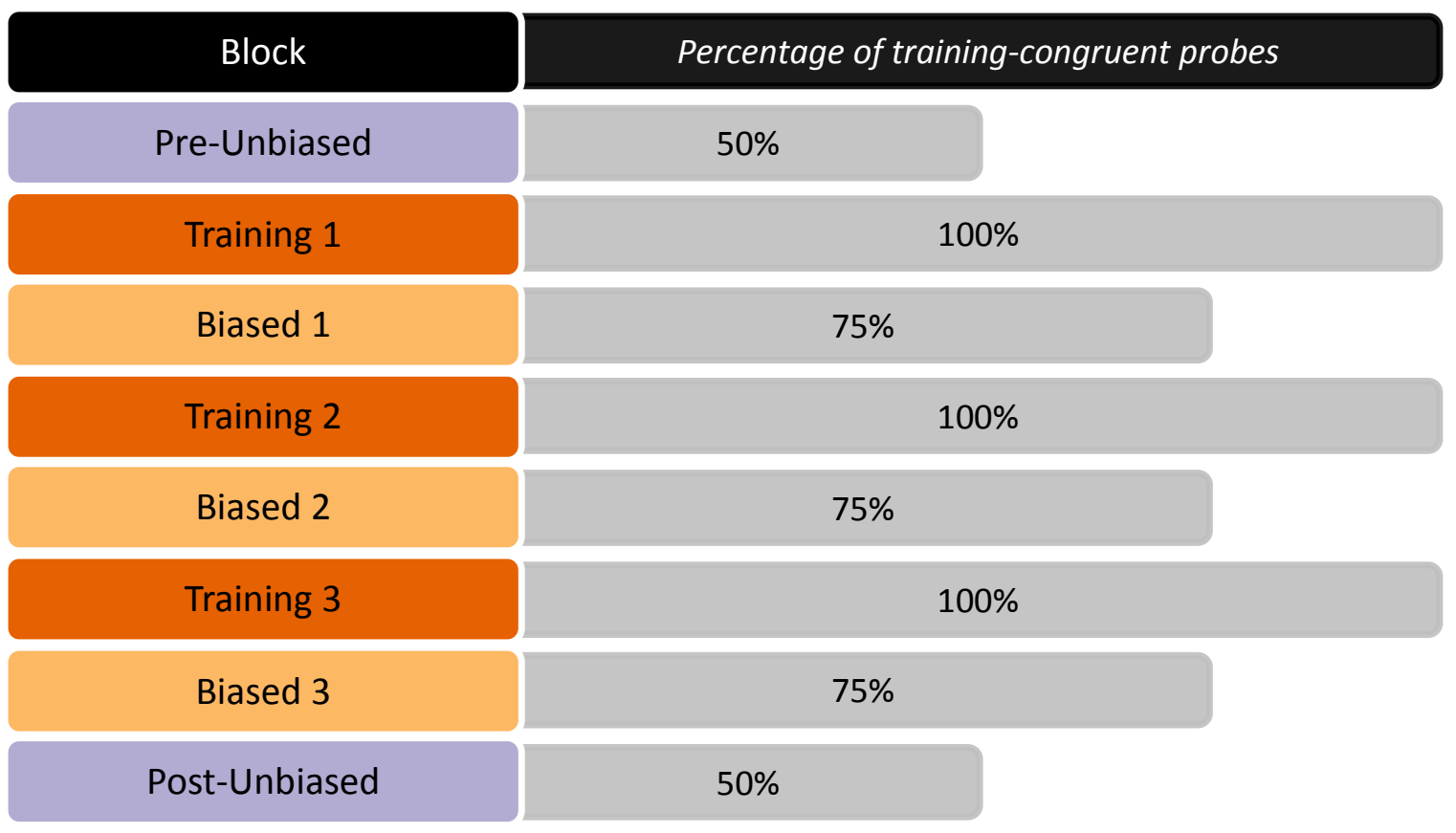

Figure 2. Block order and percentage of training-congruent probes in the ABM task in Experiment 1a. 'Training-congruent probes' were neutral probes in the neutral training condition and angry probes in the angry training condition. Each unbiased block consisted of 48 trials. Each training and biased block consisted of 96 trials.

\section{Apparatus}

All tasks were presented via a Dell Precision T1600 computer running E-Prime 2.0 (Schneider, Eschman, \& Zuccolotto, 2002a, 2002b) on a 23" Alienware 2310 monitor with a vertical refresh rate of $60 \mathrm{~Hz}$. Participants were seated with a viewing distance of approximately $60 \mathrm{~cm}$ from the monitor.

\section{Stimuli}

The ABM task used angry and neutral face pairs of six male actors from the NimStim set of facial expressions (Tottenham et al., 2009), adapted by Foster (2013). The faces were selected for raters' high accuracy in labelling the intended expression. These images were greyscaled, trimmed to only include the face and hair, and were superimposed on a grey rectangle, so that the point between the eyes was at the centre of the rectangle. Images in each pair were then matched on average pixel luminance and root mean square contrast with Image Processing Toolbox for MATLAB (The MathWorks, Natick, MA). Figure 3 provides an example of a face pair after processing. 

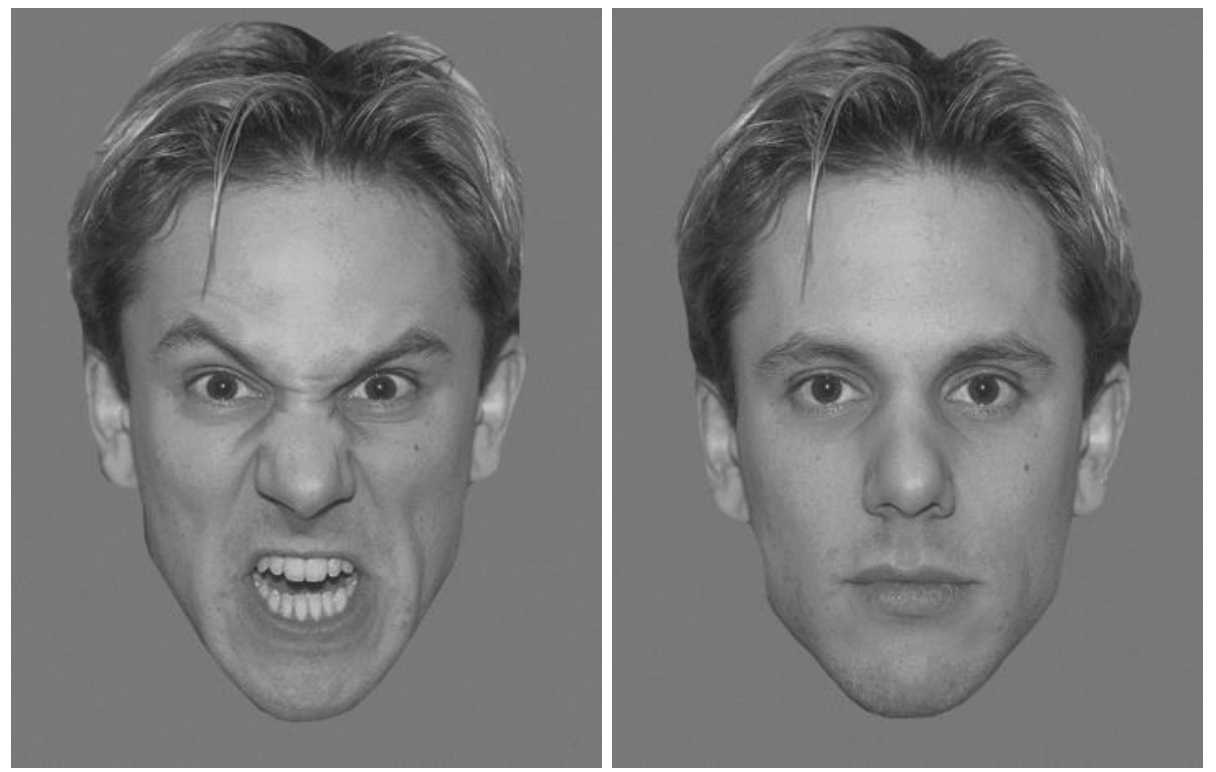

Figure 3. Example of a face pair presented in the ABM task.

\section{Procedure}

The experiment took approximately one hour to complete. Participants first completed the Mini-DASS (Lovibond \& Lovibond, 1995), Mini-MASQ (Clark \& Watson, 1991), and PSWQ (Meyer, Miller, Metzger, \& Borkovec, 1990). They then completed an ABM task. Finally, participants were verbally debriefed and received a written debriefing about the study.

ABM Task. The ABM task was a variation of the modified-dot probe task (Figure 4; Pourtois, Grandjean, Sander, \& Vuilleumier, 2004). A central white fixation cross $\left(1.5^{\circ} \times\right.$ $1.5^{\circ}$; each bar was $0.1^{\circ}$ thick) was presented on a black background for a random duration between $400 \mathrm{~ms}$ and $800 \mathrm{~ms}$. Participants were instructed to maintain fixation throughout the course of the experiment. Two faces (one angry, one neutral), each subtending $9.0^{\circ} \times 11.6^{\circ}$, one on either side of the fixation cross, were then presented for $500 \mathrm{~ms}$. At the offset of the faces a white horizontal $\left(1.5^{\circ} \times 0.3^{\circ}\right)$ or vertical $\left(0.3^{\circ} \times 1.5^{\circ}\right)$ bar probe appeared, centred on the location of one of the preceding faces; simultaneously one of the bars of the fixation cross thickened to $0.3^{\circ}$. The face stimuli and the probe were centred $8.6^{\circ}$ to the left and right of the centre of the screen and $4.1^{\circ}$ below the centre of the screen ${ }^{1}$. Participants judged whether the probe orientation was the same or different to the orientation of the thickened bar of the

\footnotetext{
${ }^{1}$ The ABM task here would later be used in Experiment 2, which investigated the effects of ABM on the N2pc component. Faces were presented in the lower visual field because this location produces larger responses at occipito-parietal sites at which the N2pc is maximal (Perron et al., 2009).
} 


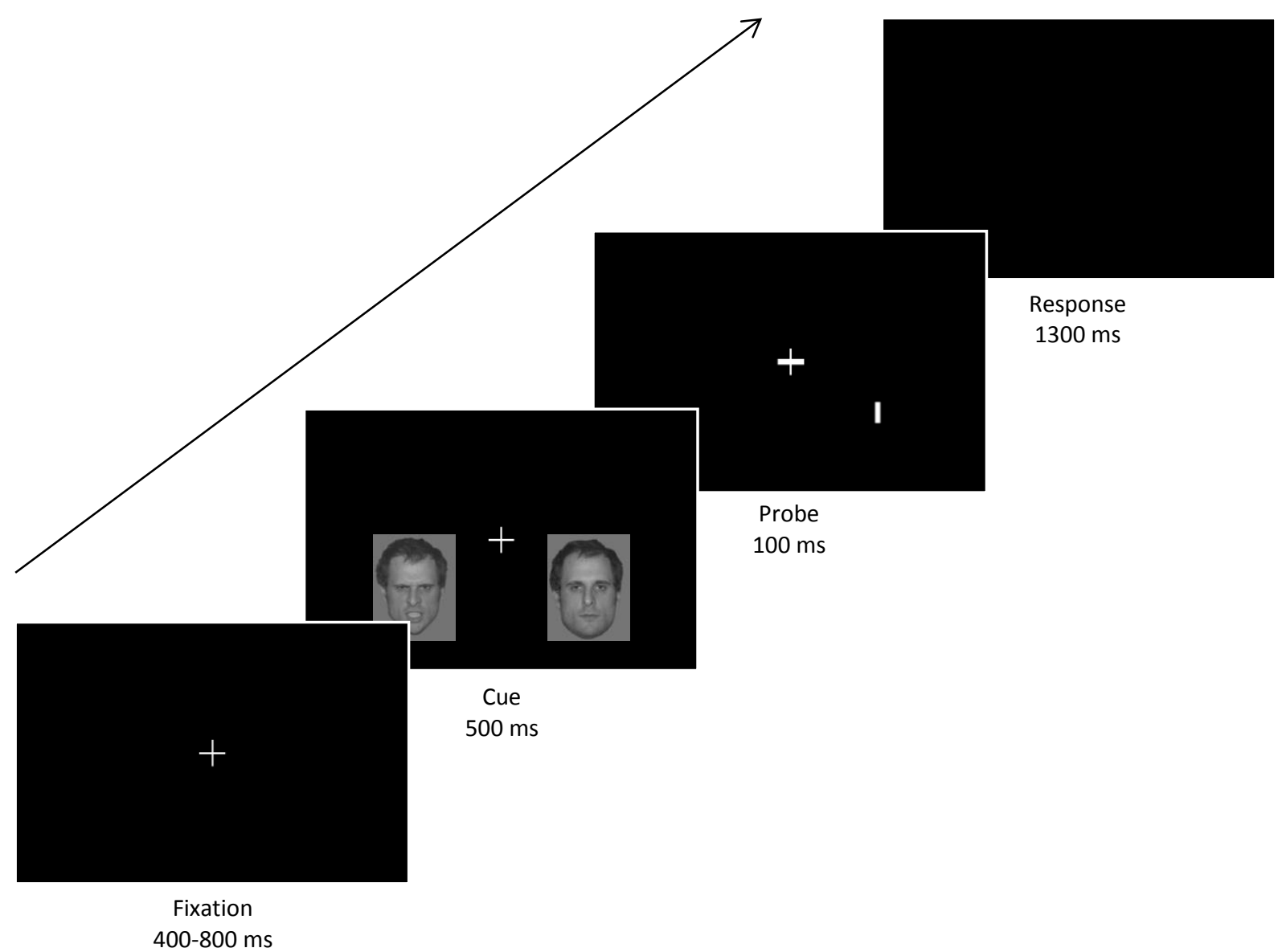

Figure 4. Sequence of events in an ABM trial in Experiment 1a. The correct response to the probe display would be 'different'.

fixation $\operatorname{cross}^{2}$, responding with 's' (for 'same') on the keyboard with the index finger of the right hand if both were vertical or both were horizontal, or with ' $d$ ' (for 'different') with the middle finger of the right hand if one was horizontal and one was vertical. The probe remained on screen for $100 \mathrm{~ms}$, followed by a blank screen for $1300 \mathrm{~ms}$, allowing a total of $1400 \mathrm{~ms}$ from the onset of the probe for a response.

Figure 2 shows the order of the blocks and the proportion of training-congruent trials in each block. The trial order within each block was randomised. The independent variables were probe location (angry, neutral) and block, both manipulated within-subjects, and training condition (angry, neutral), manipulated between-subjects.

The practice block consisted of each unique combination of emotional face location (left, right), probe location (angry, neutral), and probe discrimination (same, different) for each of the six actors $(2 \times 2 \times 2 \times 6=48$ trials $)$. The duration of the probe and the response

\footnotetext{
${ }^{2}$ Requiring participants to make a judgement about both the fixation cross and the probe should encourage them to maintain fixation when the probe appears, thus minimising lateralised eye movements. Lateralised eye movements can confound the ERP analyses used in Experiment 2.
} 
window was increased at the start of the practice trials while participants learned the task, and gradually decreased to the duration of an experimental trial (1400 ms). Participants repeated the practice block a second time if they had low accuracy on the first practice block or if they did not feel confident with the task.

In the pre- and post-unbiased blocks the probe appeared an equal number of times in the location of the preceding angry face (called the angry probe) or the neutral face (called the neutral probe). Each unbiased block consisted of each unique combination of emotional face location (left, right), probe location (angry, neutral), and probe discrimination (same, different) for each of the six actors $(2 \times 2 \times 2 \times 6=48$ trials $)$. The training blocks consisted of $100 \%$ training-congruent probes, with four repetitions of each unique combination of emotional face location (left, right) and probe discrimination (same, different) for each of the six actors $(4 \times 2 \times 2 \times 6=96$ trials). Each biased block consisted of $75 \%$ training-congruent probes, with each unique combination of emotional face location (left, right) and probe discrimination (same, different) for each of the six actors, and three repetitions with training-congruent probes and one repetition with training-incongruent probes $(2 \times 2 \times 6 \times 4$ repetitions $=96$ trials; 72 training-congruent and 24 training-incongruent). Participants thus completed a total of 672 experimental trials.

\section{Results}

\section{Questionnaires}

There were no significant differences in questionnaire scores between the two training conditions: Mini-DASS, $t(38)=0.87, p=.391$; Mini-MASQ, $t(37)=0.03, p=.979$; PSWQ, $t(36.01)=0.03, p=.974$.

\section{RTs}

Incorrect trials and trials with RTs of less than $200 \mathrm{~ms}$ were excluded from analysis (6.9\% of all trials). The remaining trials were used to calculate a median RT in each training condition. Table 1 reports the mean and SD of participants' median RTs to angry probes and neutral probes as a function of block and training condition.

Biases could not be assessed in the training blocks because RTs to both angry and neutral probes are required to calculate a bias score. Thus, analyses were conducted with the pre-unbiased block, three biased blocks, and post-unbiased block. For all analyses in which Mauchly's (1940) test of sphericity was violated, degrees of freedom were corrected using the Greenhouse-Geisser (1959) estimate of sphericity. 


\section{Table 1}

Mean (SD) of response times as a function of block and probe location in angry and neutral training conditions in Experiment 1 .

Training condition

Angry Training

Neutral Training

\begin{tabular}{lcccc}
\multicolumn{1}{c}{ Block } & Angry Probe & Neutral Probe & Angry Probe & Neutral Probe \\
\hline Pre-Unbiased & $714(88)$ & $696(69)$ & $691(51)$ & $694(67)$ \\
Biased 1 & $669(58)$ & $681(71)$ & $664(56)$ & $659(53)$ \\
Biased 2 & $643(67)$ & $640(63)$ & $650(54)$ & $654(66)$ \\
Biased 3 & $629(64)$ & $634(59)$ & $637(55)$ & $644(59)$ \\
Post-Unbiased & $624(61)$ & $624(67)$ & $631(66)$ & $631(61)$ \\
\hline
\end{tabular}

To examine the effect of training on bias across the ABM task, median RTs were analysed in a 5 (block: pre-unbiased, three biased blocks, post-unbiased) $\times 2$ (probe location: angry, neutral) $\times 2$ (training condition: angry, neutral) repeated-measures ANOVA with block and probe location as within-subject variables and training condition as the betweensubjects variable. This analysis revealed a significant main effect of block, $F(2.38,88.06)=$ $29.72, p<.001, \eta_{p}{ }^{2}=.445$, indicating that RTs decreased over the course of the experiment.

As I was interested primarily in the change in bias across blocks and the effect of training on bias, further analyses used bias scores instead of median RTs. For each participant, bias scores for each block were calculated by subtracting the median RT to angry probes from the median RT to neutral probes. Thus, a positive bias score indicated a bias toward angry faces while a negative bias score indicated a bias toward neutral faces. Figure 5 presents these bias scores as a function of block and training condition.

Bias scores were analysed in a 5 (block) $\times 2$ (training condition) repeated-measures ANOVA with block as the within-subjects factor and training condition as the between-subjects factor. This analysis revealed no block by training condition interaction, $F(3.11,115.04)=1.15, p=.335, \eta_{p}^{2}=.049$, suggesting that bias scores between the two training conditions did not differ across blocks. This null result may have been driven by the pre- and post-unbiased blocks, since no effects of training were expected during these blocks. If biases shifted in the training-congruent direction and then reverted back to baseline at the post-unbiased block, a U-shaped (quadratic) interaction would be expected. This pattern was not found; planned contrasts revealed no significant quadratic block by training condition interaction, $F(1,37)=1.30, p=.263, \eta_{p}^{2}=.034$. 


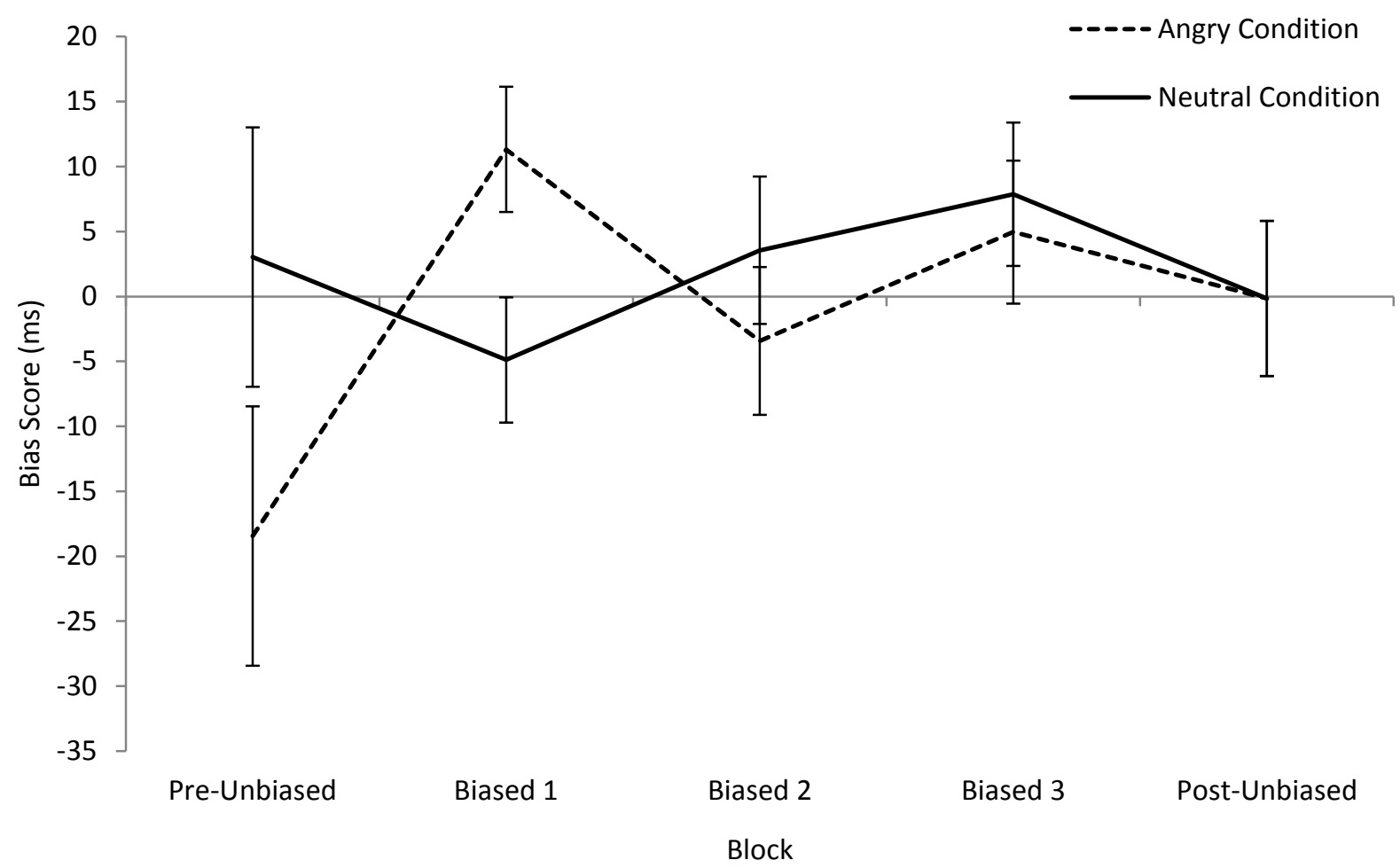

Figure 5. Bias scores as a function of block and training condition in Experiment 1a. Positive values indicate a bias toward angry faces. Error bars represent standard error of the mean.

Since Experiment 1a was exploratory, and the goal was to develop an ABM task that could then be transferred to an ERP study (Experiment 2), further post-hoc analyses were conducted to investigate whether bias scores differed between the training conditions at any point in the experiment. Independent samples $t$-tests between bias scores in the angry and neutral training condition were conducted for each block. These analyses revealed differences in two blocks. Participants in the angry training condition had a marginally greater bias toward neutral faces in the pre-unbiased block, $t(37)=1.66, p=.106, d=0.532^{3}$, and a significantly greater bias toward angry faces in the first biased block, $t(37)=-2.08, p=.045$, $d=0.667$, compared to participants in the neutral training condition. There were no significant differences between the training conditions in any other blocks. This finding suggests that participants acquired a training-congruent bias after one training block, but that this bias disappeared in later blocks.

To determine whether this effect in the first biased block was driven by the angry or neutral training condition (or both), paired-samples $t$-tests between bias scores in the preunbiased and first biased block were conducted for each training condition. This analysis

\footnotetext{
${ }^{3}$ Cohen's $d$ s for all independent-samples $t$-tests were calculated by dividing the difference between the means by the pooled standard deviation (Dunlap, Cortina, Vaslow, \& Burke, 1996).
} 
revealed a significant shift in bias toward angry faces in the angry training condition, $t(20)=$ $2.49, p=.022, d=0.892^{4}$, but no change in bias in the neutral training condition, $t(17)=$ $0.60, p=.554, d=0.238$. This finding suggests that the difference between the training conditions in the first biased block was driven by participants in the angry training condition acquiring a bias toward angry faces. However, the marginal difference in bias scores between the two training conditions in the pre-unbiased block complicates interpretation, since it may be that individuals with more extreme biases are more easily trained (Suway et al., 2013) or that changes in bias are a result of regression to the mean (e.g., O'Toole \& Dennis, 2012).

Accuracy. Table 2 reports the mean and SD of participants' accuracy as a function of probe location, block, and training condition. The 5 (block) $\times 2$ (probe location) $\times 2$ (training condition) ANOVA was conducted again with accuracy as the dependent variable. There was no main effect of block, $F(4,148)=0.40, p=.812, \eta_{p}{ }^{2}=.011$, nor a block by location by training condition interaction, $F(4,148)=0.95, p=.438, \eta_{p}{ }^{2}=.025$, or quadratic interaction, $F(1,37)=0.19, p=.662, \eta_{p}{ }^{2}=.005$. This finding indicates that accuracy did not differ by block, training condition, or probe location. Therefore, any effects in RTs or bias scores were not likely to be driven by a speed-accuracy trade-off.

Table 2

Mean (SD) accuracy to angry and neutral probes as a function of block and probe location in angry and neutral training conditions in Experiment 1 .

Training condition

Angry Training Neutral Training

\begin{tabular}{lcccc}
\multicolumn{1}{c}{ Block } & Angry Probe & Neutral Probe & Angry Probe & Neutral Probe \\
\hline Pre-Unbiased & $0.94(0.06)$ & $0.94(0.06)$ & $0.93(0.05)$ & $0.94(0.06)$ \\
Biased 1 & $0.92(0.05)$ & $0.94(0.07)$ & $0.94(0.06)$ & $0.94(0.04)$ \\
Biased 2 & $0.93(0.07)$ & $0.93(0.10)$ & $0.94(0.07)$ & $0.94(0.03)$ \\
Biased 3 & $0.93(0.09)$ & $0.91(0.13)$ & $0.92(0.08)$ & $0.94(0.08)$ \\
Post-Unbiased & $0.93(0.08)$ & $0.90(0.10)$ & $0.94(0.07)$ & $0.94(0.04)$ \\
\hline
\end{tabular}

\footnotetext{
${ }^{4}$ Cohen's $d$ s for all paired-samples $t$-tests were calculated by dividing the difference between the means by the standard deviation of the difference score (Dunlap et al., 1996).
} 


\section{Summary}

In Experiment 1a, I predicted that participants would show a training-congruent bias in biased blocks consisting of $75 \%$ training-congruent probes. This prediction was not supported; exploratory analyses showed that participants in the angry training condition acquired a bias toward angry faces after one block of training, but that this bias disappeared in later blocks. There was no shift in bias in the neutral training condition. These null effects may have been driven by participants improving at the task over time to the extent that their performance was not limited by attentional constraints. The significant decrease in RTs over the course of the task supports this hypothesis. It may have been that after the first biased block - the only block in which a training-congruent bias was observed - participants improved at the task sufficiently to discriminate the probe without needing to focus their attention on it. If participants distributed their attention broadly across the whole screen but could still discriminate the probe, they would not have needed to attend to the trained face (since there would be no advantage in detecting and discriminating the probe) and they would not have shown a bias even during the biased blocks. Alternatively, the lack of bias may be due to floor effects, that is, that participants were so fast at responding to the probe in later blocks that no difference between RTs to the angry and neutral probes could be observed.

\section{Experiment 1b}

Exploratory analysis of Experiment 1a provided some tentative evidence for a trained bias (albeit short-lived) in the ABM task. The disappearance of the trained bias in Experiment 1a may have been related to attention strategies or to floor effects. Either way, increasing the attentional demands of the task should reduce the influence of these effects, resulting in a training-congruent bias in all of the biased blocks. Thus, Experiment $1 \mathrm{~b}$ replicated Experiment 1a, but modified the probe so that it was harder to discriminate. I expected that increasing the attentional demands of the task would lead to a more robust training-congruent bias.

\section{Method}

\section{Participants}

Sixty-six participants were recruited with the same criteria as in Experiment 1a. A series of questionnaires were administered to assess participants' mood and experience with depression and anxiety. Mean scores on the Mini-DASS were 26.7 (SD = 19.6, range $=0-84$ ), on the Mini-MASQ were 50.3 (SD = 15.0, range = 29-95), and on the PSWQ were 50.2 (SD $=12.8$, range $=29-77)$. Participants were excluded from analyses if they had a total score 
greater than two SDs from the mean on any questionnaire $(n=6)$, for failing to learn the task after repeating the practice block twice $(n=2)$ or for low accuracy $(<50 \%$ in any block) in the ABM task $(n=1)$. The remaining participants (mean age: 18.5 years, $\mathrm{SD}=1.19$, range $=$ 17-23) were randomly assigned to either an angry training condition $(n=26)$ or a neutral training condition $(n=31)$.

\section{Apparatus, Stimuli, and Procedure}

The task and procedure were the same as in Experiment 1a, with some modifications to make the probe harder to discriminate (Figure 6). The probe image was changed to a grating (subtending $1.3^{\circ} \times 1.3^{\circ}$; each grating bar was $0.1^{\circ}$ thick) and appeared for $50 \mathrm{~ms}$ rather than $100 \mathrm{~ms}$. The luminance of the fixation and probe stimuli was $50 \%$ darker than in Experiment $1 \mathrm{a}^{5}$. The participants' task was to determine whether the orientation of the thickened bar of the fixation cross was the same as the orientation of the grating. To compensate somewhat for increased difficulty, the duration of the blank screen increased to $1650 \mathrm{~ms}$, allowing participants $1700 \mathrm{~ms}$ to respond. The initial speed of the practice trials was decreased accordingly while participants learned the task.

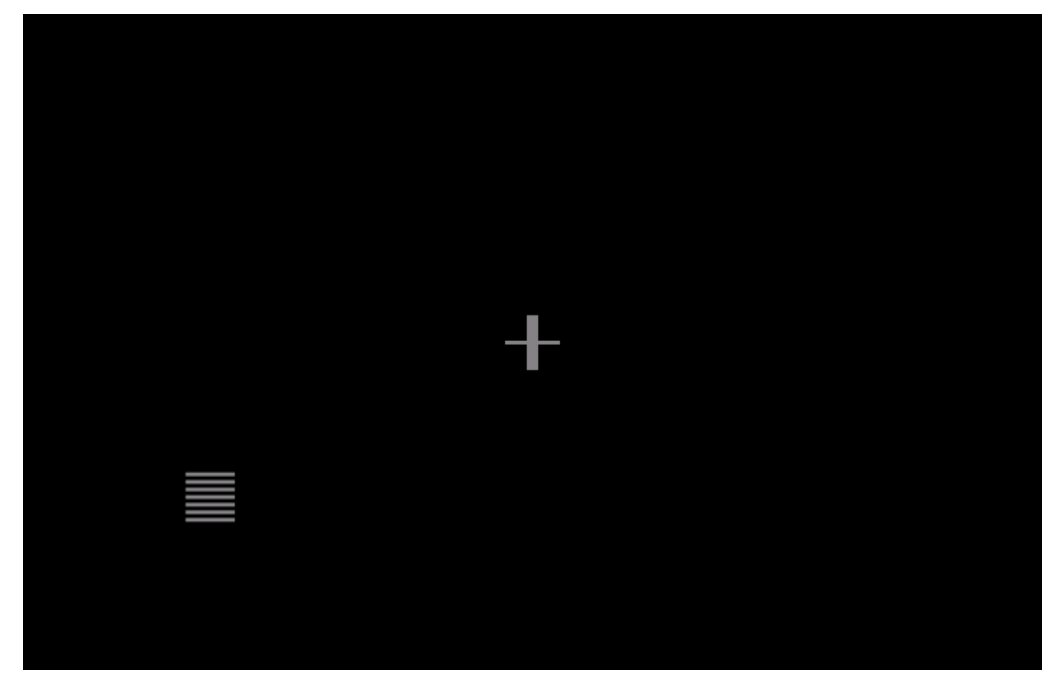

Figure 6. Example of a probe display in Experiment 1b. The correct response to this display would be 'different'.

\footnotetext{
${ }^{5}$ Feedback from participants in Experiment 1a was that the fixation and probe stimuli were too bright, so luminance was decreased for participants' comfort. In addition, decreasing the luminance should make the probe harder to discriminate.
} 


\section{Results}

\section{Questionnaires}

There were no significant differences in questionnaire scores between the two training conditions: Mini-DASS, $t(55)=0.12, p=.906$; Mini-MASQ, $t(55)=0.02, p=.987$; PSWQ, $t(55)=0.23, p=.816$.

\section{RTs}

Data were analysed in the same way as Experiment 1a. Incorrect trials and trials with RTs of less than $200 \mathrm{~ms}$ were excluded from analysis (10.5\% of all trials). Table 3 reports the mean and SD of participants' median RTs to angry probes and neutral probes as a function of block and training condition. The 5 (block) $\times 2$ (probe location) $\times 2$ (training condition) repeated-measures ANOVA revealed a significant main effect of block, $F(2.70,148.41)=$ $92.10, p<.001, \eta_{p}{ }^{2}=.626$, indicating that RTs decreased over the course of the experiment, as in Experiment 1a.

Bias scores were again calculated for each participant. Figure 7 presents these bias scores as a function of block and training condition. A 5 (block) $\times 2$ (training condition) repeated-measures ANOVA with bias score as the dependent variable was conducted. This analysis revealed a trending block by training condition interaction, $F(3.53,193.88)=2.08, p$ $=.094, \eta_{p}{ }^{2}=.036$. As predicted in Experiment 1a, participants may have acquired a bias during the biased but not the unbiased blocks. Supporting this prediction, planned contrasts revealed a significant quadratic block by training condition interaction, $F(1,55)=4.54, p$ $=.038, \eta_{p}{ }^{2}=.076$.

Table 3

Mean (SD) of response times as a function of block and probe location in angry and neutral training conditions in Experiment $1 b$.

Training condition

Angry Training

Neutral Training

\begin{tabular}{lcccc}
\multicolumn{1}{c}{ Block } & Angry Probe & Neutral Probe & Angry Probe & Neutral Probe \\
\hline Pre-Unbiased & $898(128)$ & $882(118)$ & $882(108)$ & $890(119)$ \\
Biased 1 & $793(99)$ & $801(105)$ & $794(107)$ & $778(116)$ \\
Biased 2 & $753(93)$ & $759(125)$ & $740(87)$ & $737(96)$ \\
Biased 3 & $729(91)$ & $743(98)$ & $742(111)$ & $729(102)$ \\
Post-Unbiased & $747(113)$ & $729(101)$ & $719(92)$ & $703(89)$
\end{tabular}




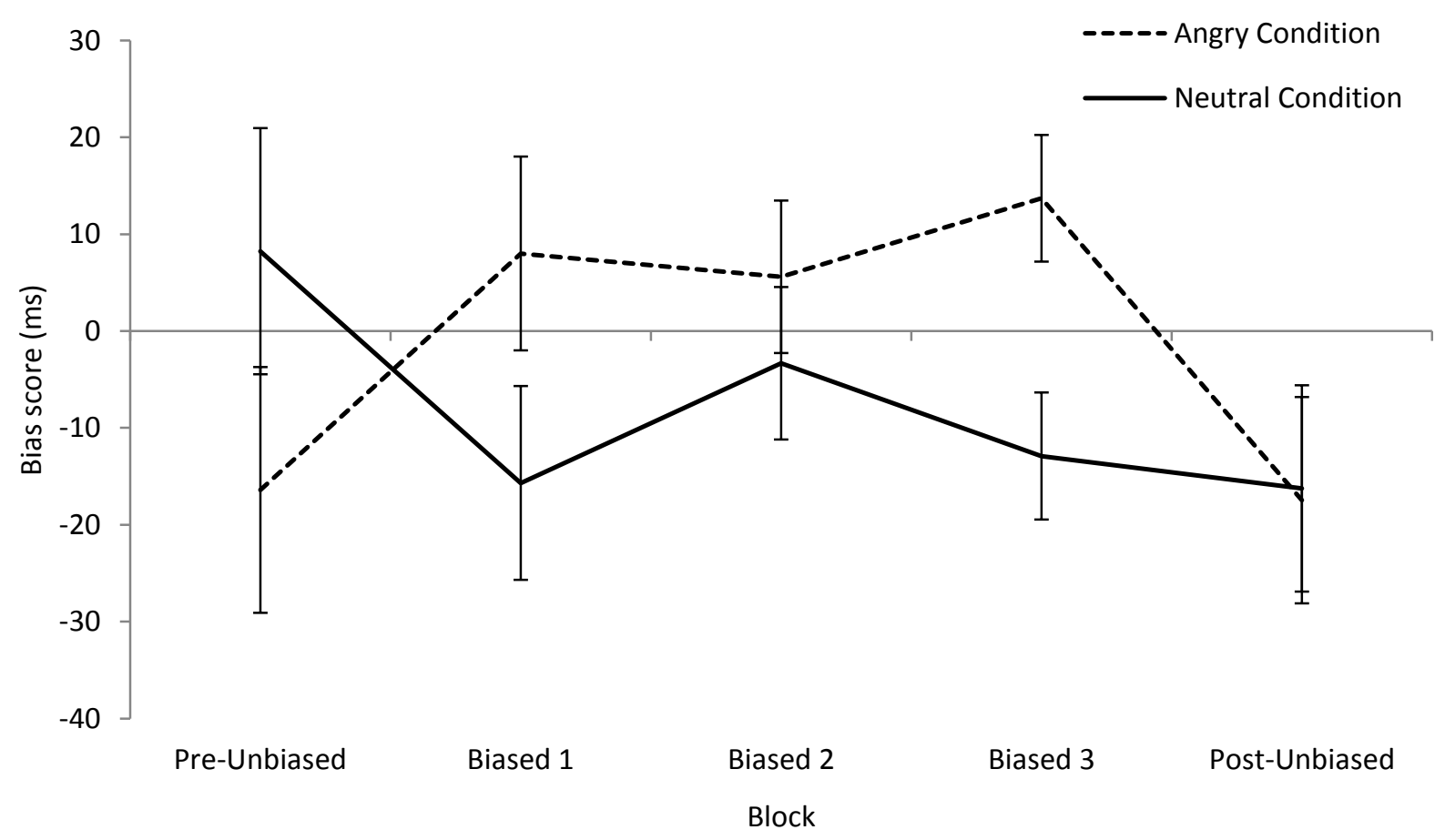

Figure 7. Bias scores as a function of block and training condition in Experiment 1b. Positive values indicate a bias toward angry faces. Error bars represent standard error of the mean.

To follow up this interaction, independent samples $t$-tests between bias scores in the training conditions were conducted for each block. These analyses revealed that participants in the angry training condition had a marginally greater bias toward angry faces in the first biased block, $t(55)=1.82, p=.074, d=-0.484$, and a significantly greater bias toward angry faces in the third biased block, $t(55)=2.50, p=.017, d=0.653$, compared to participants in the neutral training condition. There were no significant differences between the training conditions in any other blocks.

To determine whether the training effect in the first biased block was driven by changes in the angry or neutral training condition (or both), paired-samples $t$-tests between bias scores in the pre-unbiased and first biased block were conducted for each training condition. This analysis revealed a significant increase in bias toward angry faces in the angry training condition, $t(25)=2.24, p=.034, d=-0.530$, but no change in bias in the neutral training condition, $t(30)=1.67, p=.104, d=0.376$. These paired-samples $t$-tests were conducted again between bias scores in the pre-unbiased and third biased block. This analysis revealed a significant increase in bias toward angry faces in the angry training condition, $t(25)=2.23, p=.029, d=-0.618$, and a marginally significant increase in bias toward neutral faces in the neutral training condition, $t(30)=1.79, p=.084, d=0.376$. These findings 
suggest that the difference in bias scores between the two training conditions was largely driven by participants in the angry training condition acquiring a bias toward angry faces, consistent with Experiment 1a, although neutral training also appeared to have a marginal effect. However, in contrast to Experiment 1a, the trained bias persisted across the biased blocks.

\section{Accuracy}

Table 4 reports the mean and SD of participants' accuracy to the probes as a function of probe location, block, and training condition. The 5 (block) $\times 2$ (probe location) $\times 2$ (training condition) ANOVA was conducted again with accuracy as the dependent variable. There was a significant main effect of block, $F(2.52,138.31)=4.20, p=.011, \eta_{p}{ }^{2}=.071$, indicating that accuracy increased over the course of the experiment. There was no block by location by training condition interaction, $F(4,220)=.36, p=.839, \eta_{p}{ }^{2}=.006$, nor quadratic interaction, $F(1,55)=0.81, p=.373, \eta_{p}{ }^{2}=.014$. This finding indicates that accuracy to the probes did not differ by training condition or probe location. Therefore, any effects in RTs or bias scores were not likely to be driven by a speed-accuracy trade-off.

Table 4

Mean (SD) accuracy to angry and neutral probes as a function of block and probe location in angry and neutral training conditions in Experiment $1 b$.

Training condition

Angry Training

Neutral Training

\begin{tabular}{lcccc}
\multicolumn{1}{c}{ Block } & Angry Probe & Neutral Probe & Angry Probe & Neutral Probe \\
\hline Pre-Unbiased & $0.88(0.08)$ & $0.86(0.11)$ & $0.88(0.11)$ & $0.85(0.12)$ \\
Biased 1 & $0.90(0.09)$ & $0.90(0.11)$ & $0.89(0.10)$ & $0.89(0.09)$ \\
Biased 2 & $0.92(0.08)$ & $0.92(0.10)$ & $0.92(0.08)$ & $0.92(0.08)$ \\
Biased 3 & $0.91(0.10)$ & $0.90(0.14)$ & $0.89(0.11)$ & $0.89(0.10)$ \\
Post-Unbiased & $0.90(0.13)$ & $0.91(0.10)$ & $0.90(0.09)$ & $0.88(0.12)$ \\
\hline
\end{tabular}

\section{Summary of Experiments 1a and $1 \mathrm{~b}$}

In Experiment 1a, participants completed a modified $\mathrm{ABM}$ procedure. I predicted that participants would show a training-congruent bias during biased blocks in which it was advantageous for them to continue to attend to the training-congruent face. Although participants in the angry training condition acquired a bias toward angry faces following one block of training, this bias disappeared after the first biased block. The disappearance of the 
trained bias may have been related to attention strategies or to floor effects. To address these issues, Experiment $1 \mathrm{~b}$ replicated Experiment 1a but increased the attentional demands of the task by making the probe harder to discriminate. If this modification was effective at increasing the attentional demands of the task, a bias should have been observed in all of the biased blocks. Indeed, in Experiment $1 \mathrm{~b}$, participants in the angry training condition acquired a bias toward angry faces after one block of training, and this bias remained during the biased blocks before disappearing in the post-unbiased block. A similar but marginal effect was observed in the neutral training condition.

The results from Experiment 1a and 1b provide insight into the unreliable training effects in non-anxious individuals described in the ABM literature. First, because biases cannot be assessed during training, biases have typically been assessed with blocks containing 50\% training-congruent probes. No change in bias has been interpreted as ABM having no effect on the threat bias. However, the results here suggest that participants do acquire and maintain a training-congruent bias when it is still advantageous for them to maintain this bias (i.e., when the probe still appears more often in the location of the training-congruent face). The participants' ability to strategically control their attention in this way is consistent with hypotheses that suggest that non-anxious individuals have good attentional control (Eysenck et al., 2007). To the best of my knowledge, the current experiments are the first to assess changes in the threat bias in this way and show that nonanxious people are sensitive to training contingencies in ABM.

In addition, the current results suggest that the attentional demands of the task are important for training and testing a bias. Experiment 1a used probe stimuli that were more typical of the ABM literature, but only trained a short-lived bias. By reducing the probe discriminability to increase the attentional demands of the task, Experiment $1 \mathrm{~b}$ was successful in training a bias. Finally, both Experiment 1a and 1b showed that an angry bias can be trained in healthy participants after only one block (96 trials) of training.

\section{Experiment 2}

Experiment $1 \mathrm{~b}$ confirmed that the ABM task could train a bias in non-anxious participants. However, behavioural tasks like those used in Experiment $1 \mathrm{a}$ and $1 \mathrm{~b}$ cannot assess the threat bias during training because RTs to both angry and neutral probes are required to calculate a bias score. In addition, a bias score cannot differentiate between the contribution of engagement and disengagement mechanisms to the bias. To address these 
issues, the goal of Experiment 2 was to investigate the effects of ABM on ERPs to angry and neutral faces before, during, and after training.

Few studies have investigated the effects of ABM on attentional engagement and disengagement mechanisms. Of those that have, the general consensus is that ABM affects disengagement but not engagement with threat. In addition, only three studies (Eldar \& BarHaim, 2010; O’Toole \& Dennis, 2012; Suway et al., 2013) have investigated ERPs in ABM and these studies have left several areas unaddressed. First, none of these studies assessed changes in ERPs during training, relying on pre- and post-training assessments to infer changes. In addition, two of these studies (Eldar \& Bar-Haim, 2010; Suway et al., 2013) only compared responses to threat before and after ABM, without examining the effects of training on responses to neutral stimuli. Thus, their conclusions about the effects of $\mathrm{ABM}$ on the processes underlying attention to threat were only inferential. The one study (O'Toole \& Dennis, 2012) that did compare ERP responses to threat and non-threat displays used a novel variation on the ABM paradigm in which the SOA was $1000 \mathrm{~ms}$ rather than the typical 500 ms used in the ABM literature. Thus, while O'Toole and Dennis' study does provide some insight into the cognitive mechanisms underlying $\mathrm{ABM}$, it is not directly transferrable to the other literature on ABM. The goal of Experiment 2 was to address all of these issues.

Experiment 2 tested the hypothesis that $\mathrm{ABM}$ affects attentional engagement with angry faces. The ERP component that I was interested in was the N2pc, which is a robust measure of attentional engagement (see Luck, 2011, for a review). To investigate changes in engagement with angry faces (as indexed by the N2pc) during ABM, Experiment 2 replicated Experiment 1b, with some changes to the block structure. Since ERPs can show changes in processing during training blocks, and to keep the experimental session as short as possible, no biased blocks were included. In addition, two blocks of each type (pre-unbiased, training, and post-unbiased) were included to allow sufficient trials from which to reliably analyse the N2pc. Participants completed two pre-unbiased blocks (50\% training-congruent probes), three training blocks (100\% training-congruent probes), and two post-unbiased blocks (50\% training-congruent probes) (see Figure 8). Experiment 1a and 1b established that only 96 trials were necessary to train a bias. Thus, no data were analysed from the first training block in Experiment 2, when the bias was presumably being acquired. The N2pc during training was therefore calculated across the second and third training blocks.

Consistent with previous studies, I expected that participants would have a greater $\mathrm{N} 2 \mathrm{pc}$ for the angry face in the pre-unbiased blocks. If ABM affects attentional engagement, the $\mathrm{N} 2 \mathrm{pc}$ for the angry face should increase during angry training and decrease during neutral 
training. Finally, I included post-unbiased blocks to determine if the effects on the N2pc persisted beyond training.

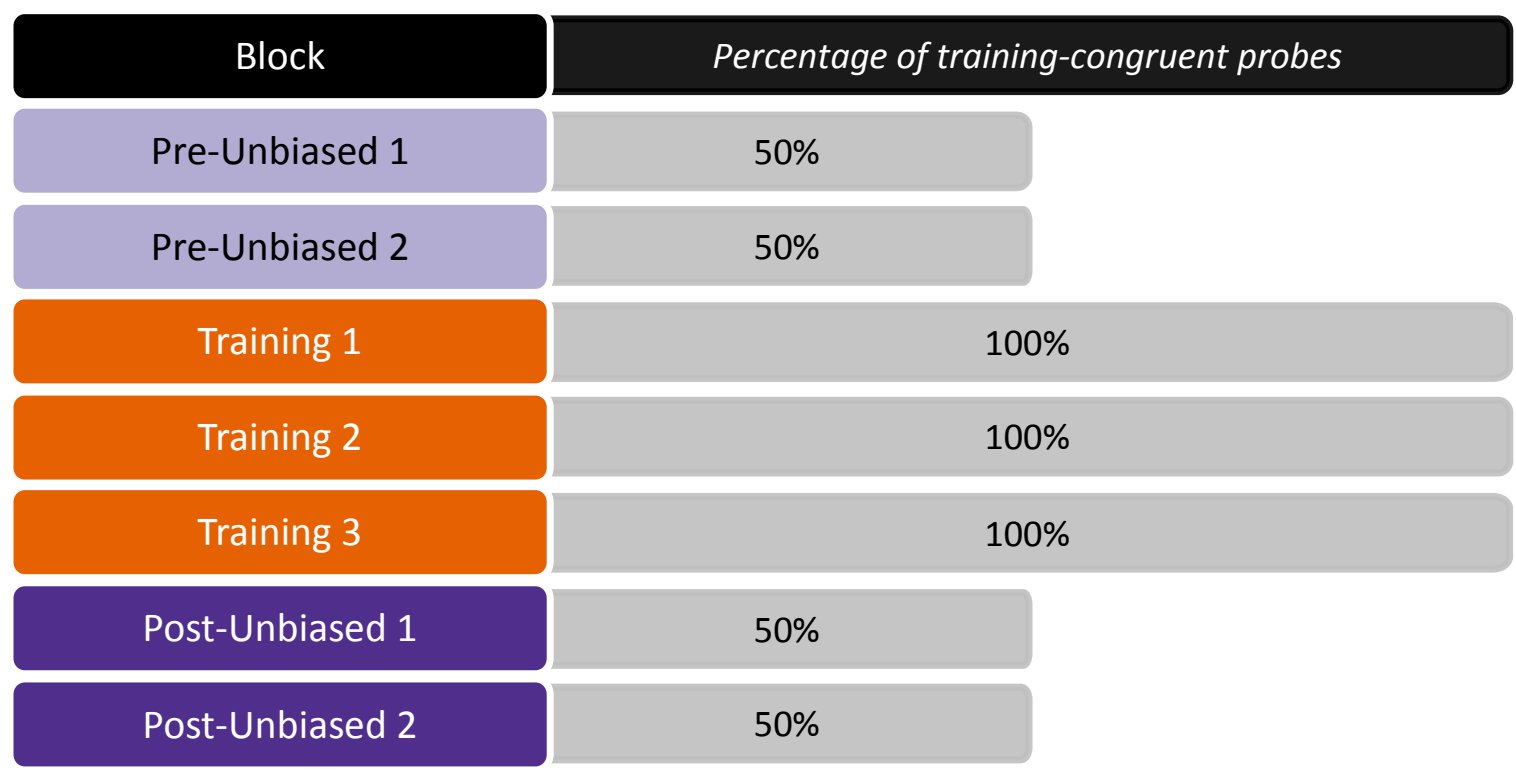

Figure 8. Block order and percentage of training-congruent probes in the ABM task in Experiment 2. Each block consisted of 96 trials.

\section{Method}

\section{Participants}

Fifty-nine participants were recruited with the same criteria as in Experiment 1a and 1b. A series of questionnaires were administered to assess participants' mood and experience with depression and anxiety. Mean scores on the Mini-DASS were 20.6 (SD = 8.0, range $=0$ 72 ), on the Mini-MASQ were 47.2 ( $\mathrm{SD}=12.8$, range $=30-85$ ), on the PSWQ were 49.3 (SD $=13.5$, range $=25-77$ ), and on the Attentional Control Scale (Derryberry \& Reed, 2002; Fajkowska \& Derryberry, 2010) were $47.0(\mathrm{SD}=4.1$, range = 36-61). Participants were excluded if they had a total score greater than two SDs from the mean on the Mini-DASS, Mini-MASQ, or PSWQ $(n=5)$. In addition, participants were excluded from analyses for neurological disorder $(n=1)$, for failing to complete the practice trials after 10 minutes $(n=$ $1)$ or for low accuracy $(<50 \%$ in any block) in the ABM task $(n=2)$. The remaining participants (mean age: 19.3 years, $\mathrm{SD}=1.80$, range $=18-26$ ) were randomly assigned to either an angry training condition $(n=23)$ or a neutral training condition $(n=27)$. Figure 9 presents a flowchart of the exclusions and analyses at each stage of the experiment. 


\section{Apparatus, Stimuli, and Procedure}

The task and procedure were the same as in Experiment 1b, with some modifications. First, the monitor vertical refresh rate was increased from $60 \mathrm{~Hz}$ to $120 \mathrm{~Hz}^{6}$. In addition to the questionnaires in Experiment 1a and 1b, participants completed the Attentional Control Scale (Derryberry \& Reed, 2002). There were also some modifications to the ABM task. The final 12 practice trials (at the same speed as the experimental trials $-1700 \mathrm{~ms}$ per trial) were repeated until participants correctly responded to at least 10 out of 12 trials. This requirement ensured that participants could complete the ABM task with a high level of accuracy before beginning the experimental trials. Following the practice trials, participants completed two pre-unbiased blocks, followed by three training blocks, and finally two post-unbiased blocks (Figure 8). A chinrest was used to keep participants at a constant viewing distance of $60 \mathrm{~cm}$ from the screen during the ABM task. The study took approximately two hours, including EEG setup, to complete.

\section{ERP Recording and Analysis}

EEG was recorded during the ABM task with a Lycra Quick-Cap (Compumedics NeuroMedical Supplies) embedded with $\mathrm{Ag} / \mathrm{AgCl}$ electrodes at 28 scalp sites (FP1, FP2, F7, F3, FZ, F4, F8, FT7, FC3, FC4, FT8, T7, C3, CZ, C4, T8, TP7, CP3, CP4, TP8, P7, P3, PZ, P4, P8, O1, Oz, and O2, according to the modified 10-20 system; American Electroencephalographic Society, 1994). To detect eye movements and blinks, the electrooculogram (EOG) was recorded from electrodes placed at the outer canthus of each eye (horizontal), and above and below the left eye (vertical). These EEG and EOG channels were referenced online to the left mastoid and re-referenced offline to the algebraic average of the left and right mastoids. Impedances were checked periodically throughout the ABM task and were kept below $10 \mathrm{k} \Omega$. The EEG signal was amplified with Professional BrainAmps and digitised using Brain-Vision Recorder software (Brain Products, Gilching, Germany). EEG and EOG were filtered online with a highpass filter of $0.02 \mathrm{~Hz}$, and digitised at a sampling rate of $500 \mathrm{~Hz}$.

\footnotetext{
${ }^{6}$ Since ERPs have a high temporal resolution, it was important to synchronise the onset of the stimuli on the monitor with the stimulus triggers in the EEG recording. A higher refresh rate increased the concordance of these two onsets.
} 


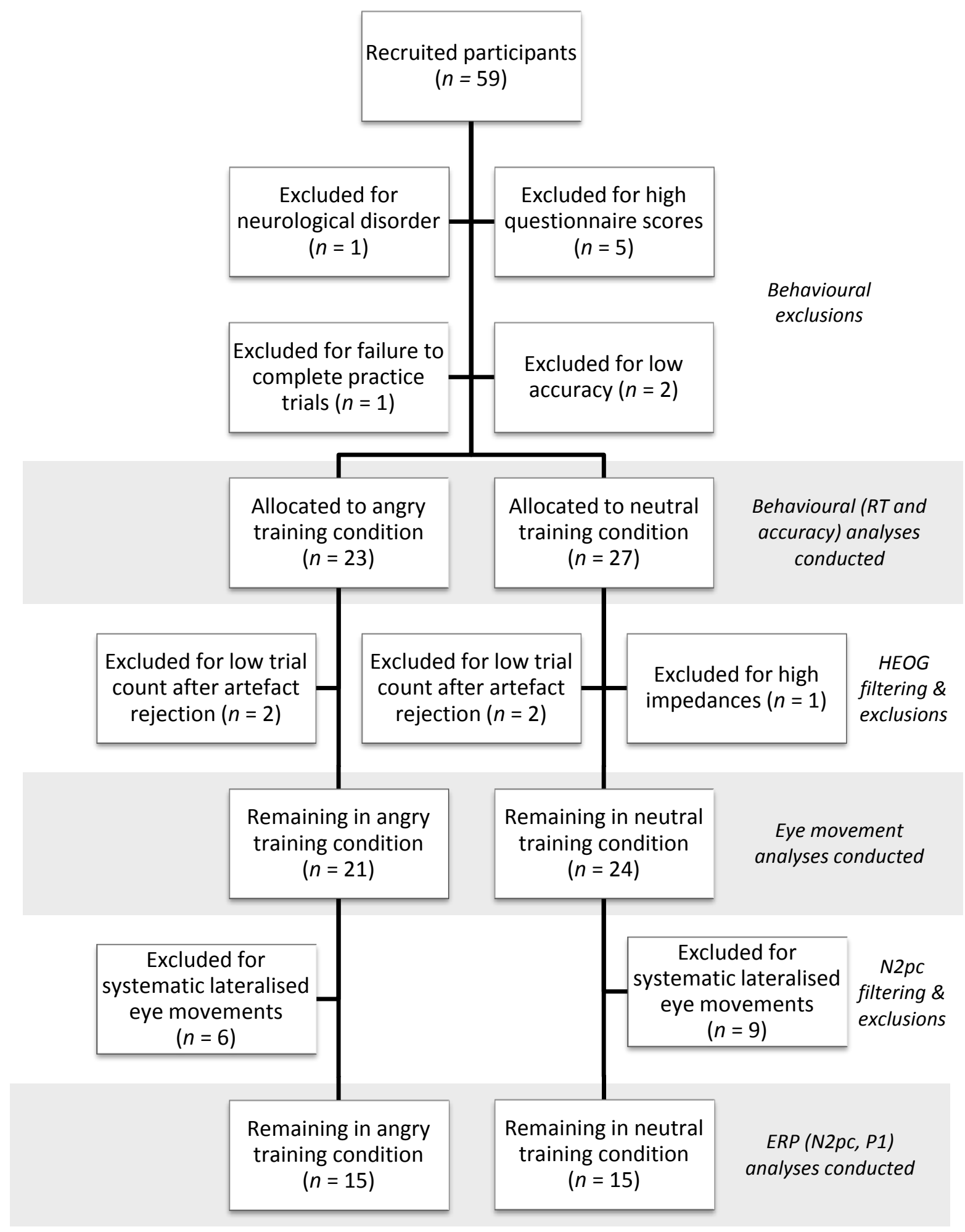

Figure 9. Participant exclusions at each stage of data analysis in Experiment 2. 
EEG data were analysed with Brain Vision Analyzer 2.0 (Brain Products, Gilching, Germany). Horizontal EOG (HEOG) was calculated by subtracting activity at the left EOG electrode from the activity at the right EOG electrode. Thus, negative values indicated a saccade to the left, and positive values indicated a saccade to the right. Similarly, the vertical EOG (VEOG) was calculated by subtracting activity at the EOG electrode above the eye from the EOG electrode below the eye. All channels were notch-filtered at $50 \mathrm{~Hz}$, and bandpass filtered from 0.1 to $30 \mathrm{~Hz}$ using a zero phase-shift Butterworth filter (12 dB/oct).

Only the pre-unbiased blocks, latter two training blocks, and the post-unbiased blocks were included in HEOG and ERP analyses. Experiment 2 was designed to assess ERP responses to the faces, but not to the probes. The probes were included solely to induce a training contingency. The EEG was thus segmented into $600 \mathrm{~ms}$ epochs, beginning $100 \mathrm{~ms}$ before the onset of the face cues, and ending at the offset of the faces (500 ms). Segments were baseline corrected by subtracting mean activity from $-100 \mathrm{~ms}$ to $0 \mathrm{~ms}$.

HEOG. For the HEOG analyses, any segment in which activity at any scalp site, VEOG, or HEOG exceeded +/- $100 \mu \mathrm{V}$ was rejected. Additionally, to remove any eye movements that occurred in the baseline period, any segment in which VEOG or HEOG activity exceeded a step of $40 \mu \mathrm{V}$ between $-100 \mathrm{~ms}$ and $0 \mathrm{~ms}$ was rejected. In addition to the behavioural exclusion criteria listed above, participants were excluded from ERP analyses if impedances exceeded $10 \mathrm{k} \Omega(n=1)$ or if more than $30 \%$ of the trials in any block $\times$ angry face location were rejected for artefacts $(n=7)$. This high rejection rate was likely due to large overt eye movements toward the training-congruent face during the training blocks, even though participants were instructed to maintain fixation. Following the first pass, 19 participants remained in the angry training condition and 23 remained in the neutral training condition. An average of $6.0 \%$ of trials were rejected for artefacts per participant (SD = 27.7, range $=2-131$ of 576 trials).

N2pc. For the N2pc analyses, it was important to remove even small systematic eye movements because the cues are no longer lateralised if horizontal eye movements are made, and the N2pc is only observed with lateralised cues. In addition, systematic eye movements can propagate back and cause lateralised activity at posterior sites (Lins, Picton, Berg, \& Scherg, 1993a, 1993b), which can confound analyses of lateralised components. First, segments in which HEOG activity exceeded $+/-50 \mu \mathrm{V}$ were rejected to minimise trials with lateralised eye movements. Then, to check for any small but systematic eye movements, average HEOG waveforms for each block $\times$ face location were calculated for each participant. Participants with a peak HEOG of $+/-3.2 \mu \mathrm{V}$ (representing a systematic eye 
movement of greater than $0.2^{\circ}$ ) during the window from $-100 \mathrm{~ms}$ to $350 \mathrm{~ms}$ were excluded from analysis $(n=12)$. This high rejection rate was likely due to systematic eye movements toward the training-congruent face during the training blocks. As a result of these exclusions, the remaining participants' systematic eye movements did not exceed $0.2^{\circ}$, with propagated voltage at posterior sites therefore not exceeding $0.1 \mu \mathrm{V}$ (Lins et al., 1993a, 1993b).

Following the second pass, 15 participants remained in each training condition, with an average of $5.1 \%$ of trials rejected per participant $(\mathrm{SD}=34.0$ range $=5-81$ of 576 trials $)$.

\section{Results and Discussion}

\section{Questionnaires}

There were no significant differences in questionnaire scores between the angry and neutral training conditions: Mini-DASS, $t(48)=0.62, p=.541$; Mini-MASQ, $t(48)=0.31, p$ $=.758 ; \mathrm{PSWQ}, t(48)=0.31, p=.858 ;$ Attentional Control Scale, $t(48)=0.81, p=.424$.

\section{Behavioural Analyses}

Participants remaining after the behavioural exclusions (Figure 9) were included in all behavioural analyses. Bias scores could only be calculated from the pre- and post-unbiased blocks, so behavioural analyses were conducted with only these blocks. For all behavioural analyses, the two pre-unbiased blocks were collapsed into one block, and the same was repeated for the two post-unbiased blocks. Analyses therefore included two blocks (preunbiased, post-unbiased) of 192 trials each.

RTs. As in Experiment 1a and 1b, incorrect trials and trials with RTs of less than 200 ms were excluded from analysis (9.0\% of all trials). The pre-unbiased blocks were then combined to calculate each participant's median RT to angry and neutral probes. This procedure was repeated for the post-unbiased blocks. Table 5 reports the mean and SD of participants' median RTs to angry and neutral probes as a function of block and training condition.

A 2 (block: pre-unbiased, post-unbiased) $\times 2$ (probe location: angry, neutral) $\times 2$ (training condition: angry, neutral) repeated-measures ANOVA was conducted with RT as the dependent variable and training condition as the within-subjects variable. A significant main effect of block, $F(1,48)=220.23, p<.001, \eta_{p}{ }^{2}=.821$, indicated that overall RTs decreased from the pre-unbiased to the post-unbiased block. Bias scores were then calculated for each for the pre- and post-unbiased blocks, and were analysed in a 2 (block) x 2 (training condition) ANOVA. As expected, there was no block by training condition interaction, $F(1$, 
48) $=0.01, p=.912, \eta_{p}{ }^{2}<.001$, indicating that there was no difference between the training conditions (Figure 10A).

Accuracy. Accuracy was analysed in the same way as RTs above. Table 6 reports the mean and SD of participants' accuracy as a function of probe location, block, and training condition. There was a significant main effect of block, $F(1,48)=8.44, p=.006, \eta_{p}{ }^{2}=.150$, and a trending main effect of location, $F(1,48)=3.73, p=.059, \eta_{p}{ }^{2}=.072$. These effects were subsumed within a significant block by location interaction, $F(1,48)=4.16, p=.047$, $\eta_{p}{ }^{2}=.080$, indicating that regardless of training condition, participants' accuracy to angry probes increased relative to their accuracy to neutral probes over the course of the experiment (Figure 10B). There was no block by location by training condition interaction, $F(1,48)=$ $1.90, p=.175, \eta_{p}{ }^{2}=.038$, indicating that the null effect in the bias scores was not driven by a speed-accuracy trade off.
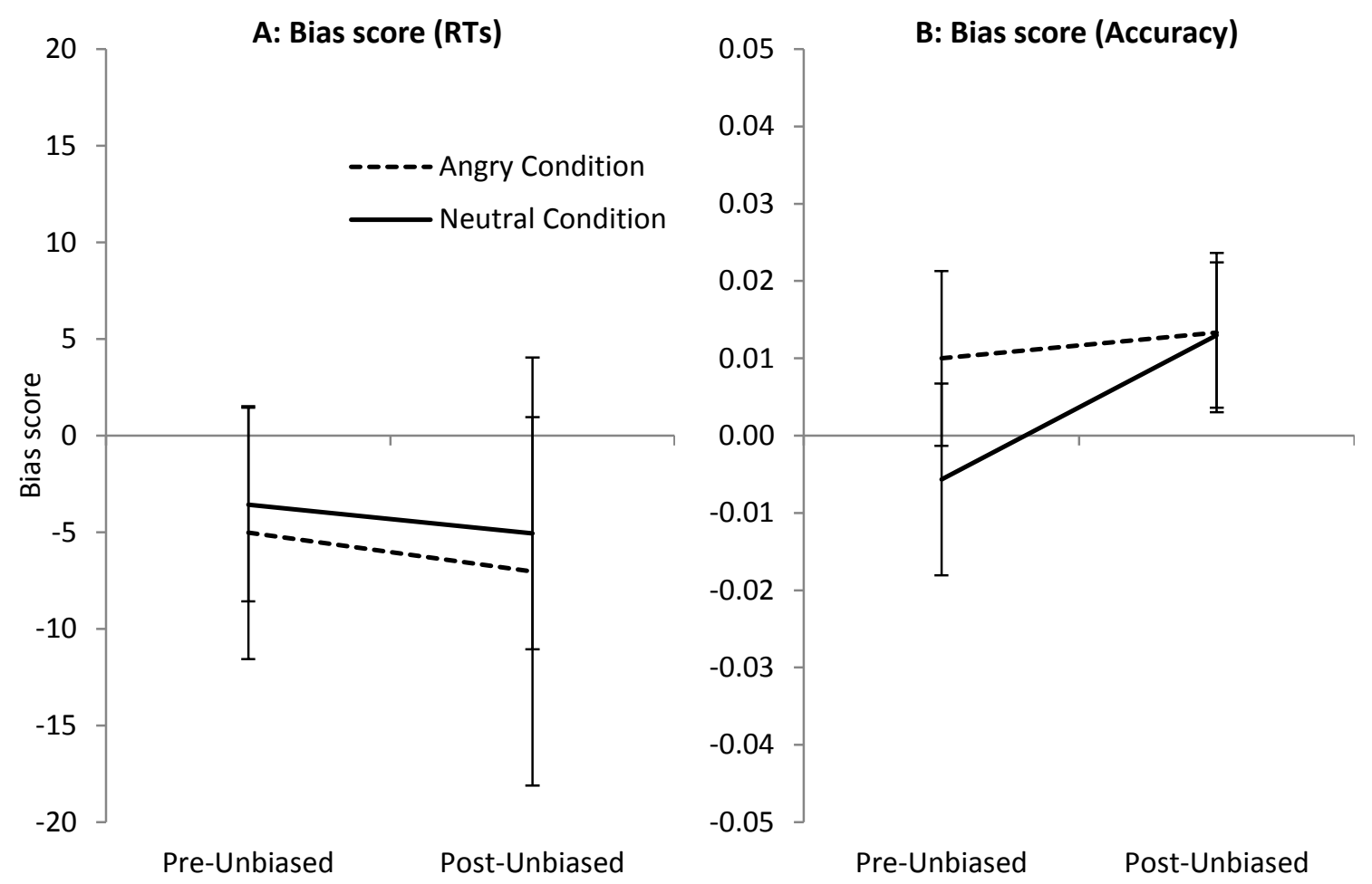

Figure 10. A: Bias scores as a function of block and training condition in Experiment 2. Positive values indicate faster responses to the angry probe (indicating an angry bias). Error bars represent standard error of the mean. B: Accuracy bias scores (accuracy to angry probe accuracy to neutral probe) as a function of block and training condition in Experiment 2. Positive values indicate that participants were more accurate at responding to the angry probe than to the neutral probe (indicating an angry bias). Error bars represent standard error of the mean. 


\section{Table 5}

Mean (SD) of RTs (ms) as a function of block and probe location in angry and neutral training conditions in Experiment 2.

Training condition

Angry Training

Neutral Training

\begin{tabular}{lcccc} 
Block & Angry Probe & Neutral Probe & Angry Probe & Neutral Probe \\
\hline Pre-Unbiased & $831(119)$ & $826(113)$ & $843(71)$ & $840(83)$ \\
Post-Unbiased & $719(94)$ & $718(92)$ & $723(60)$ & $723(54)$ \\
\hline
\end{tabular}

Table 6

Mean (SD) accuracy to angry and neutral probes as a function of block and probe location in angry and neutral training conditions in Experiment 2.

Training condition

Angry Training Neutral Training

\begin{tabular}{ccccc}
\multicolumn{1}{c}{ Block } & Angry Probe & Neutral Probe & Angry Probe & Neutral Probe \\
\hline Pre-Unbiased & $0.90(0.07)$ & $0.90(0.09)$ & $0.91(0.05)$ & $0.90(0.05)$ \\
Post-Unbiased & $0.92(0.06)$ & $0.91(0.07)$ & $0.93(0.05)$ & $0.91(0.06)$ \\
\hline
\end{tabular}

\section{ERP Analyses}

For all ERP analyses, the two pre-unbiased blocks were collapsed into one block. The same was repeated for the latter two training blocks and the two post-unbiased blocks. There were therefore three analysis blocks (pre-unbiased, training, and post-unbiased) of 192 trials each.

HEOG. The bias could not be assessed during training because RTs to both angry and neutral faces are necessary to calculate a bias score. However, some indications of participants' behavioural response to training can be inferred from their lateralised eye movements as indicated by HEOG activity. Although participants were instructed to maintain fixation throughout the ABM task, they may have made systematic eye movements when the faces appeared, particularly in training blocks in which the faces were predictive of the probe location. If participants were sensitive to the training contingency and unable to control their eye movements, I predicted that they would make systematic eye movements in the training-congruent direction during training. To test this prediction, HEOG was used to calculate an eye movement index (EMI) that indicated whether eye movements were toward or away from angry faces. In the original HEOG, positive values indicated eye movements to the right. If participants moved their eyes toward an angry face appearing on the right, the 
HEOG would be positive, and if they moved their eyes toward an angry face on the left, the HEOG would be negative. The EMI was thus calculated so that positive EMI values indicated eye movements toward angry faces. This EMI was calculated with the following formula:

$$
\frac{H E O G_{\text {Angry Right }}-H E O G_{\text {Angry Left }}}{2}
$$

Figure 11 presents the EMI as a function of block and training condition. In this first analysis, I included those participants who showed systematic eye movements even during the N2pc window. Visual inspection of this figure suggests that participants systematically moved their eyes in the training-congruent direction during the training block at approximately $350 \mathrm{~ms}$ after the face onset. To quantify this observation, a late EMI was calculated from the mean amplitude of the HEOG from $350 \mathrm{~ms}$ to $500 \mathrm{~ms}$ (Table 7). The late EMI was then subjected to a 3 (block: pre-unbiased, training, post-unbiased) $\times 2$ (training condition) repeated-measures ANOVA. This ANOVA revealed a trending block by training condition interaction, $F(2,86)=3.029, p=.054, \eta_{p}{ }^{2}=.066$. If participants only moved their eyes in the training-congruent direction during training, but not during the pre- or postunbiased blocks, a significant U-shaped (quadratic) interaction would be expected. Planned analyses confirmed a quadratic block by training condition interaction, $F(1,43)=3.46, p$ $=.025, \eta_{p}{ }^{2}=.112$.

This interaction was followed up to investigate whether the EMI differed between the angry and neutral training conditions in any block. Independent samples $t$-tests of the late EMI in the angry and neutral training conditions were conducted for each block. These analyses revealed no significant difference between the training conditions in the preunbiased block, $t(43)=-0.095, p=.925, d=-0.028$, or post-unbiased block, $t(43)=0.192, p$ $=.849, d=0.057$. There was, however, a significant difference between the training conditions in the training block, $t(40)=2.666, p=.011, d=0.797$, in which participants in the angry training condition had a positive EMI (i.e., toward angry faces) and participants in the neutral training condition had a negative EMI. These findings indicate that participants were moving their eyes in the training-congruent direction during training, suggesting that they were sensitive to the training contingency. Although a bias score could not be calculated from the training block, these results suggest that Experiment 2 had a similar training effect to that observed in Experiment 1b. In addition, participants did not move their eyes in the training-congruent direction during the unbiased blocks, which is also consistent with the 
participants in Experiment 1a and 1b showing no bias in the unbiased blocks. Notably, these eye movements occurred after the N2pc window.
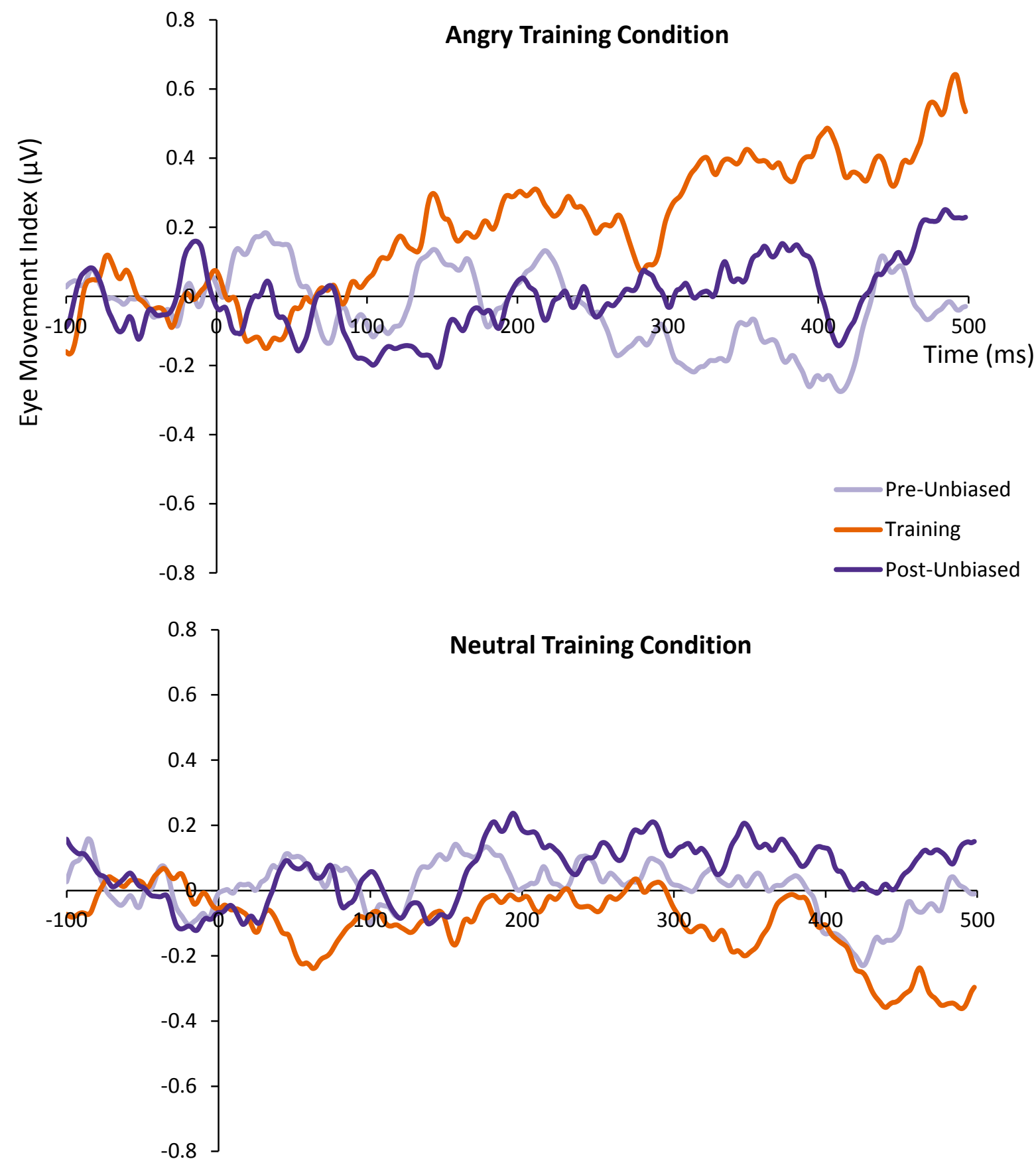

Figure 11. The eye movement index calculated from horizontal EOG in the angry training condition (top) and neutral (bottom) training condition in Experiment 2. A positive score indicates that participants were moving their eyes toward the angry face. 


\section{Table 7}

Mean (SD) of the late eye movement index ( $\mu \mathrm{V}$; quantified from $350 \mathrm{~ms}$ to $500 \mathrm{~ms}$ ) as a function of block and training condition. Positive values indicate systematic eye movements toward the angry face in Experiment 2.

Training condition

\begin{tabular}{lcc} 
Block & Angry Training & Neutral Training \\
\hline Pre-Unbiased & $-0.10(0.77)$ & $-0.08(0.66)$ \\
Training & $0.42(0.98)$ & $-2.64(0.75)$ \\
Post-Unbiased & $0.09(0.57)$ & $0.06(0.63)$ \\
\hline
\end{tabular}

N2pc. Only trials and participants remaining after N2pc filtering and exclusion stage (see Figure 9) were used to calculate the N2pc, because N2pc analyses can be confounded by lateralised eye movements. To select the time window from which the N2pc would be analysed, mean amplitudes were calculated from P7/P8 electrodes contralateral and ipsilateral to the angry face (Figure 12A). The peak of the overall (i.e., collapsed across block and training condition) difference wave of the contralateral and ipsilateral electrodes was used to guide selection of the analysis window. The N2pc window was thus defined as $180 \mathrm{~ms}$ to 320 ms after the onset of the faces, which is similar to the N2pc window defined in other studies (Brosch et al., 2011; Foster, 2013; Grimshaw et al., 2014; Mazza et al., 2009). Before analysing the $\mathrm{N} 2 \mathrm{pc}$, it was important to ensure that the additional criteria for the $\mathrm{N} 2 \mathrm{pc}$ filtering and exclusion stage were effective at minimising lateralised eye movements during the N2pc window. An early EMI from the trials remaining after the N2pc filtering stage was quantified as the mean amplitude of the HEOG during the N2pc window. This early EMI was then subjected to a 3 (block) $\times 2$ (training condition) repeated-measures ANOVA. This analysis revealed no block by training condition interaction, $F(2,56)=1.12, p=.333, \eta_{p}{ }^{2}$ $=.043$, or quadratic interaction, $F(1,28)=1.50, p=.232, \eta_{p}{ }^{2}=.059$, confirming that the remaining participants were not systematically moving their eyes in the direction of the trained face during the N2pc window (see Figure 13).

To investigate whether training affected the N2pc, mean amplitudes of the contralateral and ipsilateral electrodes between $180 \mathrm{~ms}$ and $320 \mathrm{~ms}$ (Table 8) were subjected to a 3 (block) $\times 2$ (electrode laterality) $\times 2$ (training condition) repeated-measures ANOVA. This ANOVA revealed a main effect of electrode laterality, $F(1,58)=19.0, p<.001, \eta_{p}{ }^{2}=$ .490 , indicating that the mean amplitude at electrodes contralateral to the angry face was more negative than the mean amplitude at the ipsilateral electrodes. This finding indicated 


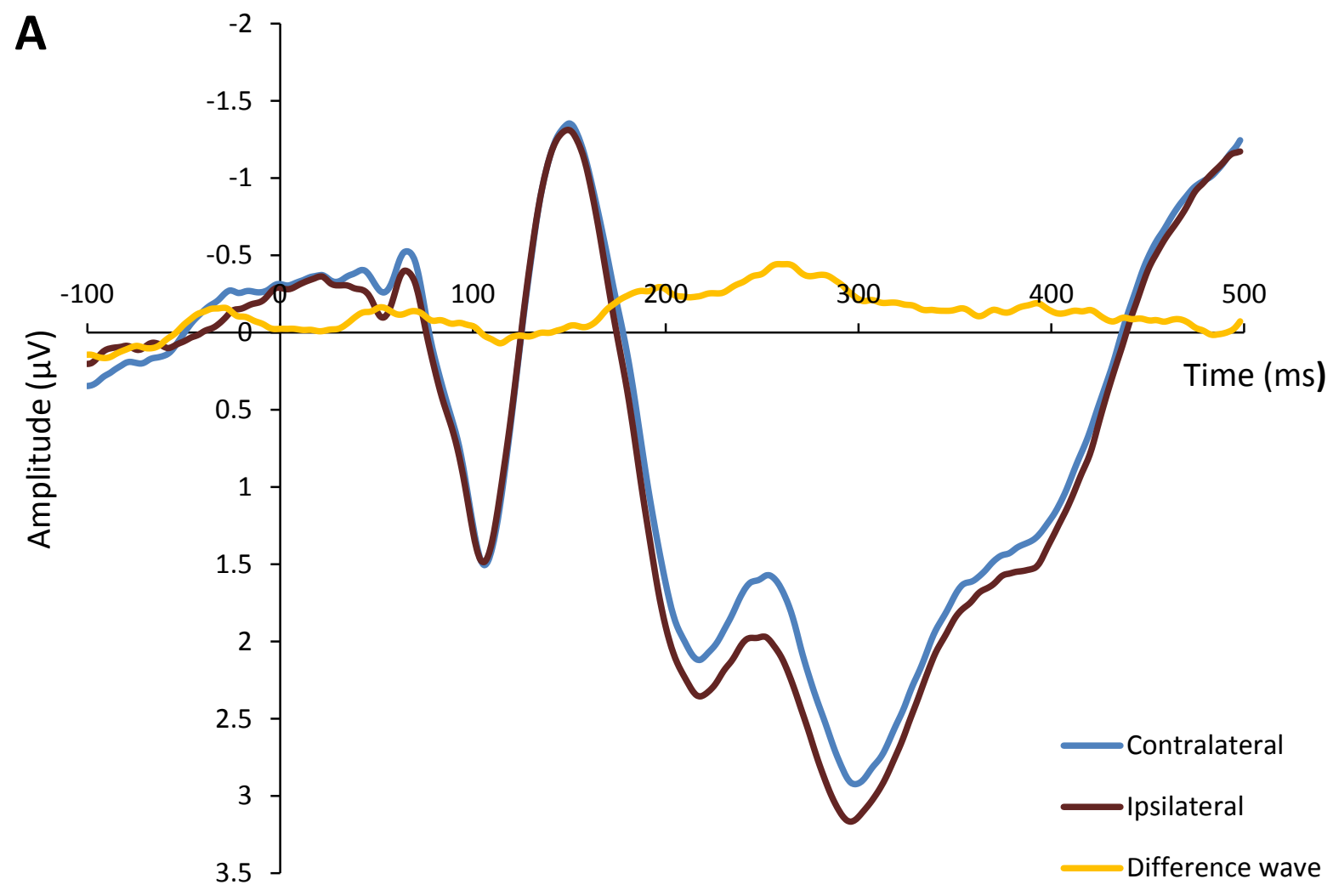

B
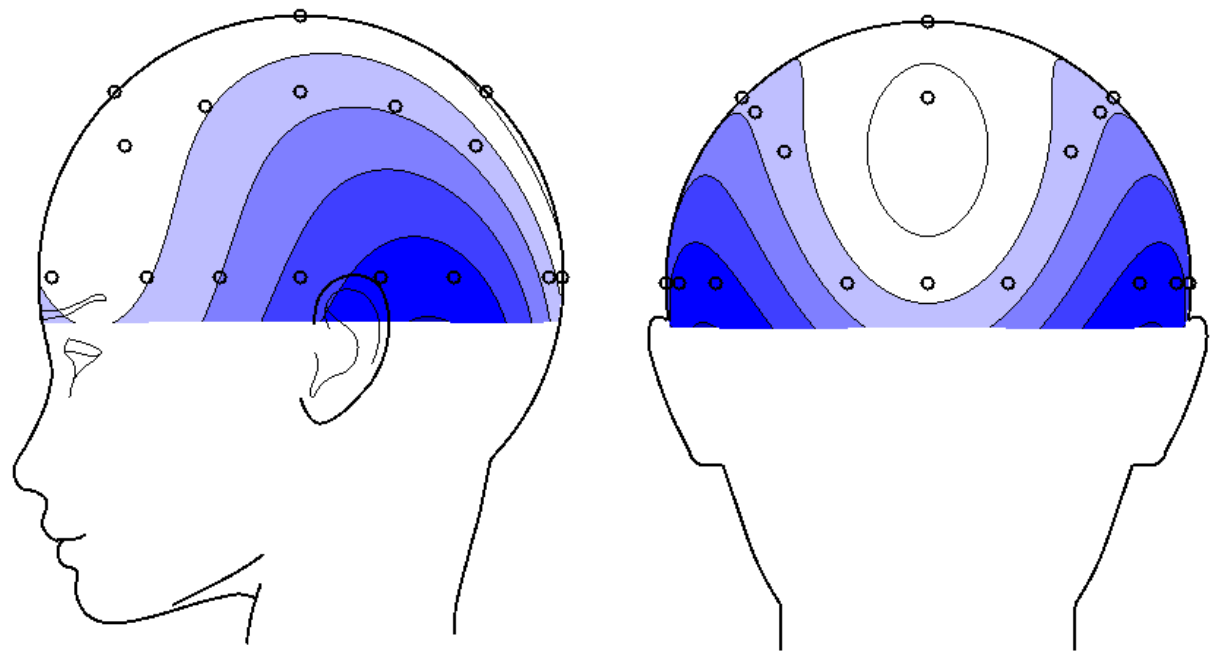

$-0.5$

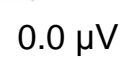

Figure 12. A: Overall N2pc measured at the $\mathrm{P} 7 / \mathrm{P} 8$ electrodes contralateral and ipsilateral to the angry face in Experiment 2. Difference wave is ipsilateral - contralateral activity. B: Scalp distribution of the N2pc difference wave during the N2pc window (180 ms to $320 \mathrm{~ms}$ ). 
that overall, participants had an N2pc for the angry face (Figure 12A). The topographic map of this $\mathrm{N} 2 \mathrm{pc}$ confirms that the $\mathrm{N} 2 \mathrm{pc}$ had the expected scalp distribution and was most negative at occipito-parietal sites (Figure 12B). This finding is consistent with previous studies that have found an N2pc for angry faces (Foster, 2013; Grimshaw, Foster, \& Corballis, 2014; Holmes, et al., in press).

If $\mathrm{ABM}$ affected engagement with the angry face, it was predicted that the N2pc would shift in a training-congruent direction during training. However, the expected block by laterality by training condition interaction was not significant, $F(2,56)=0.54, p=.583, \eta_{p}{ }^{2}$ $=.019$. As in the behavioural results, it was possible that the N2pc shifted in the training-congruent direction during the training block but not during the unbiased blocks, in which case a U-shaped (quadratic) interaction would be expected. However, planned contrasts revealed no significant quadratic interaction, $F(1,28)=0.82, p=.373, \eta_{p}{ }^{2}=.028$. These findings indicate that the N2pc did not differ by training condition or block, suggesting that attentional engagement (as reflected in the N2pc) is not influenced by ABM. Figure 14 presents this finding as the difference between contralateral and ipsilateral electrodes as a function of block and condition.

It is possible that the participants who were excluded for excessive eye movements in the early EMI (i.e., during the N2pc window) were those who were most sensitive to the training contingency, leading to the null effect of training on the N2pc. To test whether included and excluded participants were different on bias or late EMI measures, bias scores and the late EMI were re-analysed with exclusion status as an additional between-subjects factor.

First, to establish whether the included participants had different bias scores to the excluded participants, a 2 (block: pre-unbiased, post-unbiased) $\times 2$ (training condition) $\times 2$ (exclusion: included, excluded) repeated-measures ANOVA with bias score as the dependent variable and training condition and exclusion as the between-subjects variables was conducted. This analysis revealed no significant interactions with the exclusion factor, suggesting that there were no differences in bias scores between included and excluded participants.

Second, to investigate whether included participants showed a similar eye movement pattern as the excluded participants, a 3 (block: pre-unbiased, training, post-unbiased) $\times 2$ (training condition) $\times 2$ (exclusion) repeated-measures ANOVA with the late EMI (i.e., calculated from trials remaining after the HEOG filtering stage in the $350 \mathrm{~ms}$ to $500 \mathrm{~ms}$ window; see Figure 9) as the dependent variable was conducted. This analysis revealed no 
significant interactions with the exclusion factor, suggesting that there were no differences in the EMI between included and excluded participants.
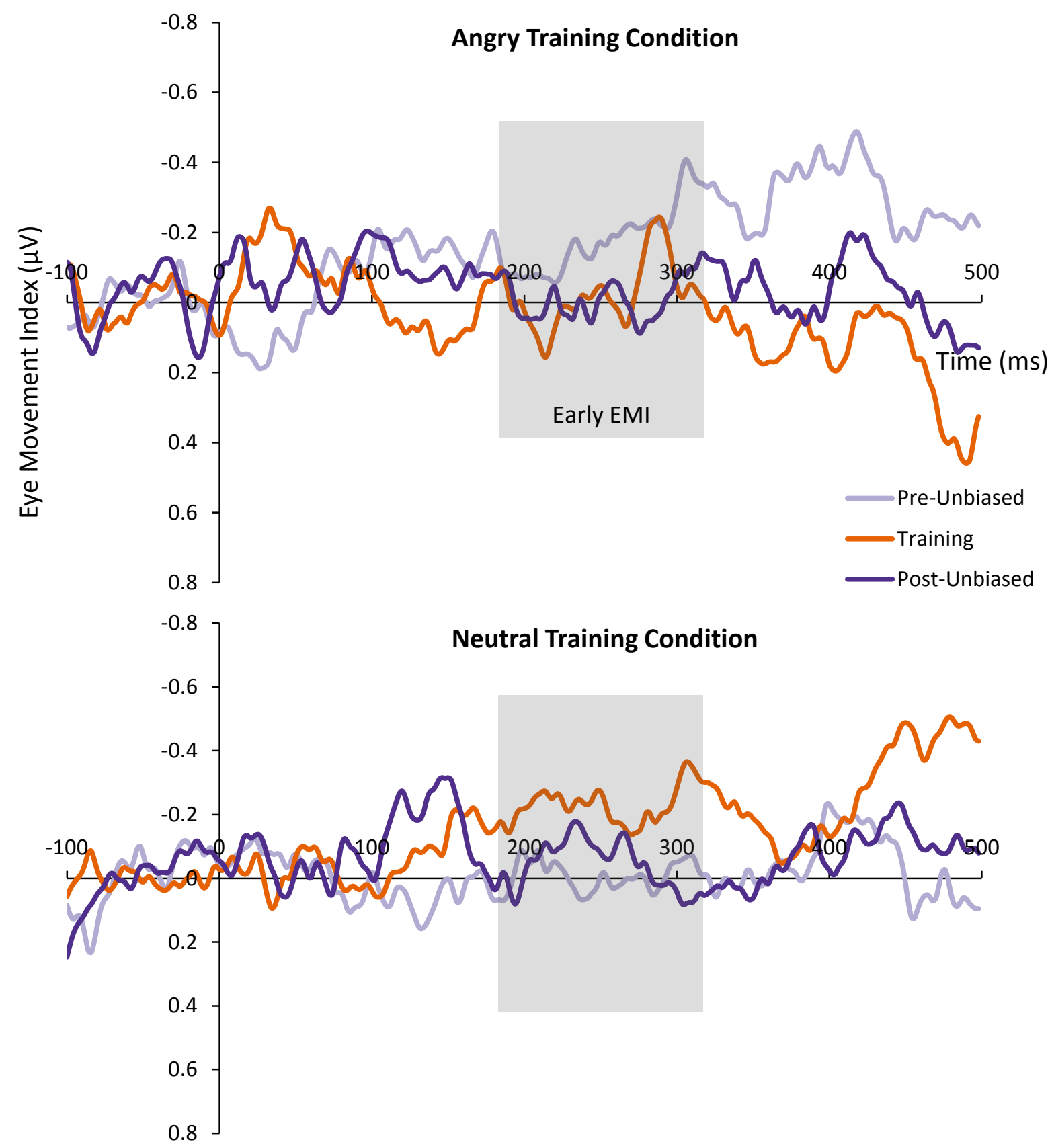

Figure 13. The eye movement index calculated from horizontal EOG in the angry (top) and neutral (bottom) training condition, calculated from trials and participants remaining after the N2pc filtering and exclusion criteria (see Figure 9) in Experiment 2. A positive score indicates that participants were systematically moving their eyes toward angry faces. 
Table 8

Mean (SD) of the amplitude $(\mu \mathrm{V})$ at P7/P8 electrodes contralateral and ipsilateral to the angry face during the N2pc window (180 ms to $320 \mathrm{~ms}$ ) as a function of block and training condition in Experiment 2.

Training condition

Angry Training Neutral Training

\begin{tabular}{lcccc}
\multicolumn{1}{c}{ Block } & Contralateral & Ipsilateral & Contralateral & Ipsilateral \\
\hline Pre-Unbiased & $2.16(1.53)$ & $2.37(1.4)$ & $2.01(2.44)$ & $2.24(2.39)$ \\
Training & $1.78(1.45)$ & $2.00(0.49)$ & $1.95(2.31)$ & $2.30(2.36)$ \\
Post-Unbiased & $1.89(1.50)$ & $2.34(1.67)$ & $2.03(2.16)$ & $2.38(2.42)$ \\
\hline
\end{tabular}

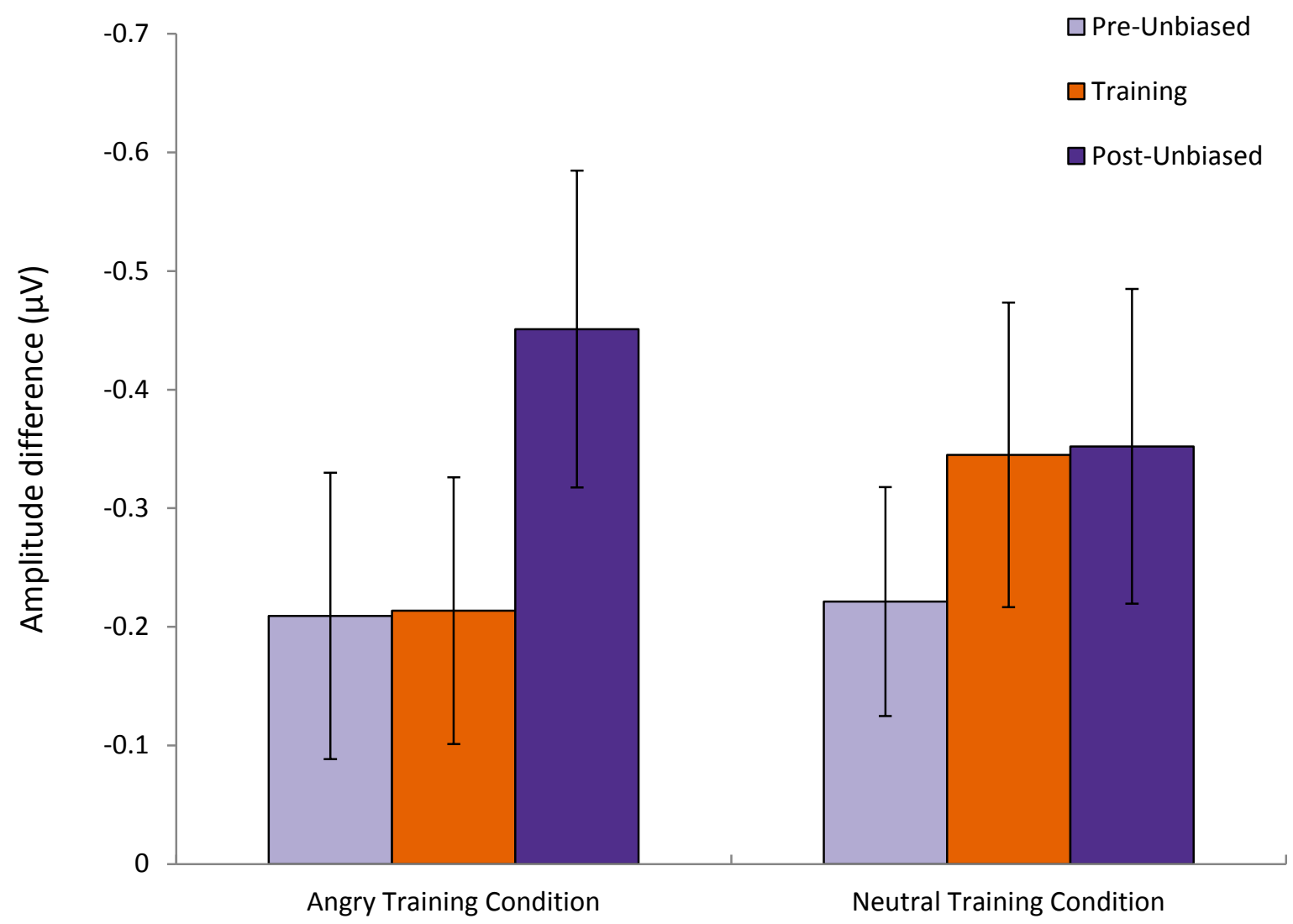

Figure 14. Difference between mean amplitude at the contralateral and ipsilateral (relative to angry face) electrodes during the N2pc time window (160 ms to $310 \mathrm{~ms}$ ) in Experiment 2. Larger negative values indicate a greater N2pc for the angry face. Error bars represent standard error of the mean. 
Taken together, these findings suggest that there were no confounding differences between the included and excluded participants in bias scores. Although included participants by definition made fewer systematic eye movements than excluded participants, these findings indicate that the included participants' eye movements in the late EMI period were not different to those of the excluded participants. Therefore, included participants were sensitive to the training contingency, and the null interaction effects in the N2pc suggest that $\mathrm{ABM}$ does not affect attentional engagement with angry faces.

Lateralised P1. The angry and neutral face pairs used in the ABM task were matched for average pixel luminance and root mean square contrast. However, because the angry and neutral faces were different by definition, not all low-level properties (e.g., spatial frequency) could be matched between the face pairs. These lower-level differences could be driving the overall N2pc for the angry face, in which case the N2pc would not be related to the threat salience of the angry face. The lateralised P1, which appears as a positive-going wave contralateral to a salient (by lower-level properties) stimulus that is receiving enhanced processing (Hillyard, Vogel, \& Luck, 1998), can be used to test this confound. If the overall $\mathrm{N} 2 \mathrm{pc}$ for the angry face was driven by lower-level sensory imbalances, a greater P1 contralateral to the angry face would be expected. To test this possibility, the lateralised P1 was calculated in the same way as the N2pc, except in an earlier time window (80 ms to 120 ms) selected from the peak of the P1 in the overall waveform (Figure 12). This P1 was then subjected to the same 3 (block) $\times 2$ (training condition) ANOVA as the N2pc. This analysis revealed no main effect of laterality, $F(1,28)=0.17, \mathrm{p}=.687, \eta_{p}{ }^{2}=.006$, suggesting that the main effect of the N2pc for the angry face was not driven by differences in lower-level properties between the angry and neutral faces. Unexpectedly, there was a significant main effect of block, $F(2,56)=5.07, \mathrm{p}=.009, \eta_{p}{ }^{2}=.153$, which was subsumed within a significant block by laterality by training condition interaction, $F(1.59,44.48)=5.036, p$ $=.016, \eta_{p}{ }^{2}=.152$, and quadratic interaction, $F(1,28)=5.947, p=.021, \eta_{p}{ }^{2}=.175$. This finding indicated that the lateralised P1 changed as a result of block and training condition, with the greatest change occurring in the training blocks. To follow up this finding, a difference measure was calculated by subtracting the ipsilateral P1 from the contralateral P1 (Table 9). Figure 15 shows the difference in mean amplitude in the P1 window as a function of block and training condition and Figure 16 presents this P1 as a difference wave. 
Table 9

Mean (SD) of the amplitude $(\mu \mathrm{V})$ at P7/P8 electrodes contralateral and ipsilateral to the angry face during the P1 window (80 ms to $120 \mathrm{~ms}$ ) as a function of block and training condition in Experiment 2.

Training condition

Angry Training Neutral Training

\begin{tabular}{lcccc}
\multicolumn{1}{c}{ Block } & Contralateral & Ipsilateral & Contralateral & Ipsilateral \\
\hline Pre-Unbiased & $0.53(1.58)$ & $0.56(1.83)$ & $0.23(1.90)$ & $0.38(1.81)$ \\
Training & $0.89(1.44)$ & $0.70(1.44)$ & $0.94(1.55)$ & $1.18(1.62)$ \\
Post-Unbiased & $0.78(1.68)$ & $0.78(1.63)$ & $0.98(1.65)$ & $0.60(1.60)$ \\
\hline
\end{tabular}

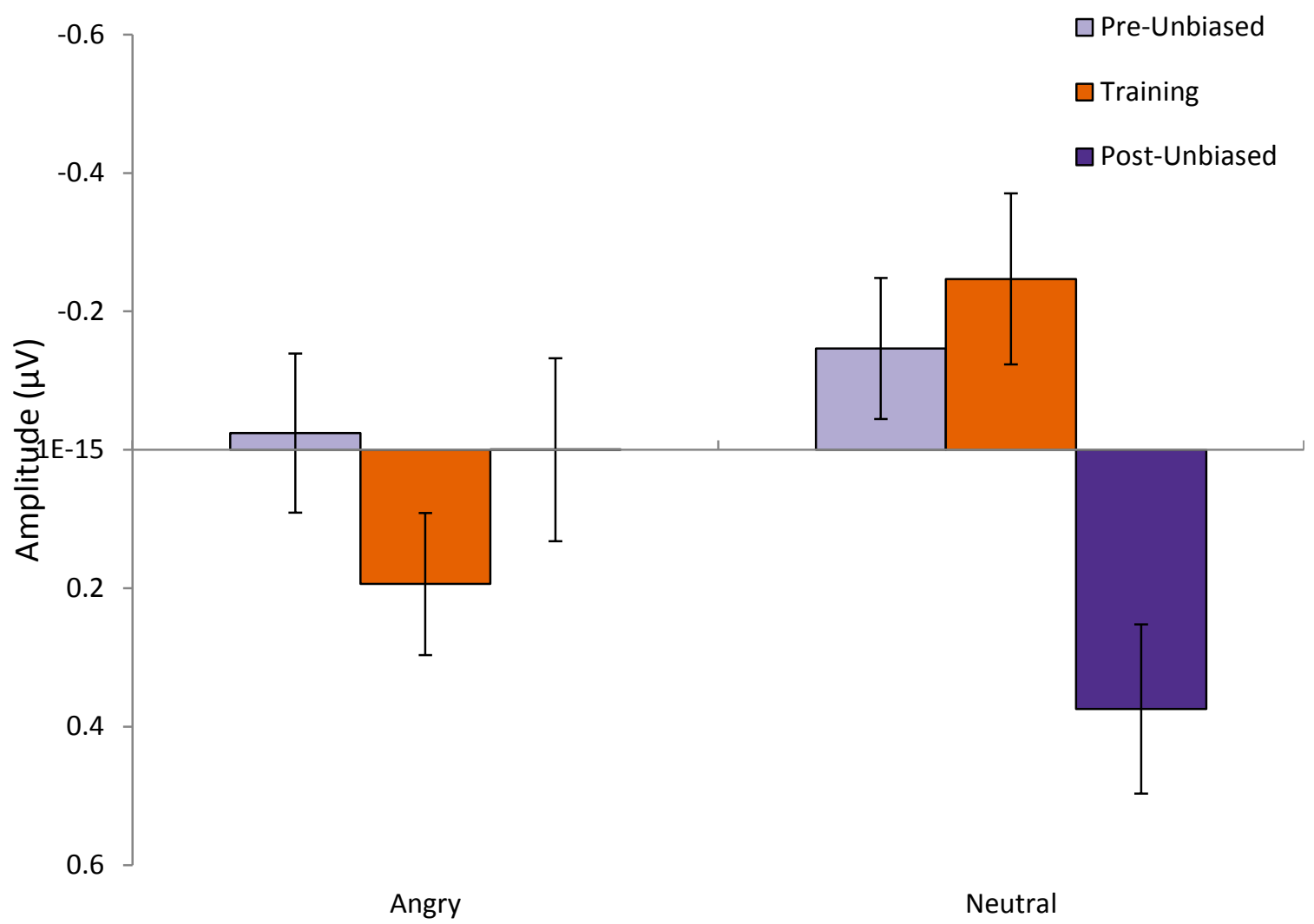

Figure 15. Difference between contralateral and ipsilateral (relative to angry face) P7/P8 electrodes in the P1 time window ( $80 \mathrm{~ms}$ to $120 \mathrm{~ms}$ ) in Experiment 2. More positive values indicate a greater contralateral P1 to the angry face. Error bars represent standard error of the mean. 

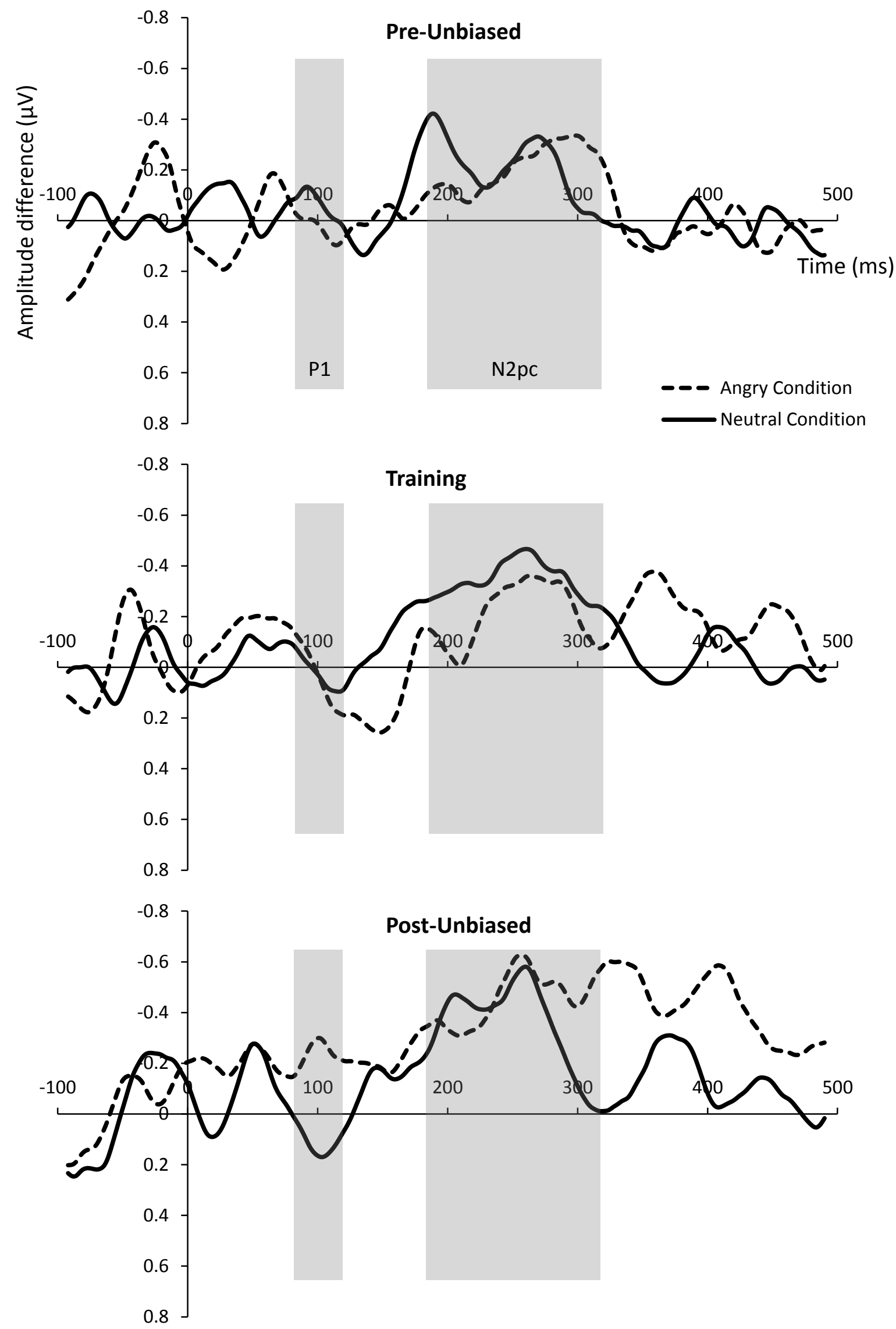

Figure 16. Contralateral - ipsilateral difference waves calculated from the grand average of each block of P7/P8 electrodes contralateral and ipsilateral to the angry face in Experiment 2. 
To investigate whether the P1 difference measure in each training condition differed in any block, independent samples $t$-tests were conducted between each training condition's P1 difference score in each block. This analysis revealed that there was no difference between the two training conditions in the pre-unbiased block, $t(28)=.656, p=.517, d=$ 0.240. Participants in the angry training condition had a marginally greater P1 contralateral to the angry face than participants in the neutral training condition during training, $t(21.9)=$ $1.961, p=.063, d=0.716$. This trend reversed in the post-unbiased block, during which participants in the neutral training condition had a significantly greater P1 contralateral to the angry face than participants in the angry training condition, $t(28)=2.259, p=.032, d$ $=-0.825$. The face cues were identical across all three blocks, so this modulation of the lateralised P1 must be related to training effects rather than to lower-level visual properties.

\section{Summary}

Experiment 2 investigated the effects of $\mathrm{ABM}$ before, during, and after training. Behavioural analyses were also conducted in the pre- and post-unbiased blocks. These analyses showed that bias scores did not change as a result of training. Participants in both the angry and the neutral condition had increased accuracy to angry probes after training. ERP analyses were conducted on HEOG activity and the N2pc and lateralised P1 components. The HEOG revealed that participants moved their eyes in the training-congruent direction during training blocks only, suggesting that they were sensitive to the training contingency. N2pc analyses showed that participants had an overall N2pc for the angry face, consistent with previous studies of N2pc for threatening faces. However, this N2pc was not modulated by block nor training condition. This finding suggests that training does not affect engagement with angry faces as indexed by the N2pc. Next, a lateralised P1 analysis confirmed that the different lower-level features of the angry and neutral faces did not underlie the overall N2pc. Unexpectedly, the P1 appeared to be modulated by block and training condition. However, this result, while interesting, was unexpected and not the primary focus of this experiment and therefore needs further follow-up before any conclusions may be drawn.

\section{General Discussion}

Anxious individuals pay more attention to threat than non-anxious individuals (BarHaim et al., 2007; Cisler, Bacon, et al., 2007; Cisler \& Koster, 2010). Recent studies have shown that training these anxious individuals to preferentially attend to neutral information reduces their threat bias and anxiety levels (Bar-Haim, 2010; Beard et al., 2012; Hakamata et 
al., 2010). However, despite the large amount of research on ABM, the majority of studies have focused on its clinical outcomes. As a result, the cognitive mechanisms that are involved in ABM are still poorly understood. In this thesis, I aimed to extend our understanding of the role of attentional engagement in the modification of the threat bias. Experiment 1 was used to develop an ABM task that could train a bias toward either angry or neutral faces in nonanxious individuals. This task was then used in Experiment 2 to investigate ERP responses to the faces before, during, and after training. These experiments showed that although ABM shifted the behavioural threat bias in the training-congruent direction, it did not alter the $\mathrm{N} 2 \mathrm{pc}$, an electrophysiological index of attentional engagement. These findings suggest that ABM does not affect engagement with threat. Instead, it seems likely that ABM reduces anxiety and the threat bias by improving participants' ability to disengage from threat.

Only non-anxious individuals were included in the current experiments because I was primarily interested in the effect of $\mathrm{ABM}$ on the engagement mechanism, which is presumably unimpaired in healthy individuals. One potential problem with this approach is that non-anxious participants do not always show a reliable attentional bias (Cooper \& Langton, 2006), and they do not always show effects of training (Eldar \& Bar-Haim, 2010; O'Toole \& Dennis, 2012). However, this unreliability may be an artefact of the way that the threat bias is assessed. A bias score is calculated from the difference between RTs to probes appearing in the location of threat cues and probes appearing in the location of neutral cues. Thus, a bias cannot be assessed during training when the probe always appears in the location one of the cues. As a result, the threat bias is typically measured in pre- and post-training assessment blocks in which the probe appears equally often in the location of the threat or the neutral cue. Non-anxious participants may acquire a bias toward the training-congruent cue during training, but this bias may revert to baseline in the post-training assessment when it is no longer advantageous to maintain a training-congruent bias. Therefore, in Experiment 1, the typical ABM task was modified to include 'biased' blocks containing $75 \%$ training-congruent probes (see Figure 2 for the block structure). Thus, a bias score could be calculated from RTs in the biased blocks, while it remained advantageous for participants to attend to the training-congruent faces.

In Experiment 1a, participants failed to acquire a training-congruent bias. Exploratory post-hoc analyses suggested that they may have acquired a training-congruent bias after only one block of 96 training trials, however the bias was short-lived, and disappeared in the later biased blocks. The disappearance of the bias was contrary to my prediction that participants would show a training-congruent bias in all of the biased blocks. Why did the bias disappear? 
Participants may have improved at the task enough to distribute their attention broadly across the whole screen and still discriminate the probe, in which case they would have shown no bias. Alternatively, the bias may have disappeared because of floor effects. Supporting this hypothesis, RTs significantly decreased over the course of the experiment. To reduce these potential attention strategy and floor effects, in Experiment 1b I replicated Experiment 1a but increased the attentional demands of the task by making the probe harder to discriminate. This manipulation was successful; participants acquired a training-congruent bias after one block of training and this bias persisted throughout the biased blocks. The bias in Experiment $1 \mathrm{~b}$ disappeared in the final post-unbiased block, when the probe appeared equally often in the location of the angry or neutral face. This finding supports the hypothesis that the unreliable effects of ABM in non-anxious individuals are an artefact of the way that the bias is assessed. The conclusions of previous studies that ABM does not modify the threat bias may have been incorrect, since in those studies participants may have acquired a bias during training but then reverted to baseline in the post-training assessment. The current results are also consistent with studies in the non-emotional attentional literature that have found that participants acquire a bias toward cues that predict the location of a probe $80 \%$ of the time (Lambert et al., 1999). Future studies of ABM in non-anxious individuals should consider testing biases with blocks in which it remains advantageous for participants to attend to the training-congruent cues.

The task from Experiment $1 \mathrm{~b}$ was then used in Experiment 2 to investigate ERP responses to the faces before, during, and after training. ERPs were advantageous to use in this experiment for two reasons: first, ERPs do not require a behavioural response, so they can be measured during training when a bias score cannot be calculated. Second, ERPs have a high temporal resolution and different components have been linked to dissociable cognitive processes, so ERP responses to the faces can be used to differentiate subtle changes in processing that arise through training. To date, only three studies have used ERPs to investigate ABM (Eldar \& Bar-Haim, 2010; O’Toole \& Dennis, 2012; Suway et al., 2013). Of these studies, none have investigated changes in ERPs during training, and none have used the N2pc, an established measure of attentional engagement. In addition, only one study has compared ERPs between threat and non-threat displays (O'Toole \& Dennis, 2012), but it used a novel ABM task, so the results are less transferrable to the ABM literature. To address these issues, in Experiment 2 I investigated the effect of ABM on the N2pc component. Participants completed a pre-unbiased block, a training block, and a post-unbiased block, and EEG was recorded throughout. If ABM affects engagement with angry faces, I expected the 
$\mathrm{N} 2 \mathrm{pc}$ for the angry face to increase during angry training and to decrease during neutral training.

In Experiment 2, behavioural bias scores did not change from the pre-unbiased to the post-unbiased blocks. This finding is consistent with Experiment 1b, in which a training-congruent bias was observed in biased but not unbiased blocks. Although bias scores could not be assessed during training in Experiment 2, eye movements toward the faces were measured from HEOG activity. Although participants were instructed to maintain fixation and not make eye movements, HEOG analyses revealed that participants moved their eyes toward the training-congruent faces during training but not during the pre- or post-unbiased blocks. Given the predictive value of the faces, it seems that it was difficult for participants to maintain fixation. These findings indicate that participants were sensitive to the training contingency, and suggest that Experiment 2 produced a similar behavioural effect to Experiment $1 \mathrm{~b}$. These findings also support the hypothesis that non-anxious participants acquire a training-congruent bias during training, but that this bias disappears in the posttraining assessment when it is no longer advantageous for them to attend to the training-congruent cue.

$\mathrm{N} 2 \mathrm{pc}$ analyses showed that participants had an overall N2pc for the angry face, consistent with previous research (Foster, 2013; Grimshaw et al., 2014; Holmes, Bradley, Kragh Nielsen, \& Mogg, 2009). However, this N2pc was not affected by training. Before drawing any conclusions from this finding, it is important to rule out alternative explanations for the null effect of training on the N2pc. First, the participants included in the N2pc analyses may have been those who were less sensitive to the training contingency, because a large number of participants were excluded from N2pc analyses for large systematic eye movements during the N2pc window. However, analyses confirmed that the included participants did not have different bias scores or late eye movements (i.e., occurring after the $\mathrm{N} 2 \mathrm{pc}$ window) compared to the excluded participants. In addition, the overall N2pc was highly significant, suggesting that there were enough participants and enough trials to assess the N2pc in each block. There was no trend in the results for the N2pc to shift in the training-congruent direction during training - if anything, the N2pc for the angry face during training was greater in those participants who received neutral training than those who received angry training (see Figure 15).

It is also possible that the overall N2pc was driven by differences in the lower-level properties of the angry and neutral faces, and not by attentional engagement with the angry faces. This possibility was unlikely, because the faces were matched for average pixel 
luminance and root mean square contrast, although it is impossible to match neutral and angry faces on all visual parameters. To test this potential confound, the lateralised P1 was analysed in Experiment 2. A greater P1 contralateral to the angry faces would indicate that the angry faces had more salient lower-level visual properties than neutral faces. This analysis revealed no overall lateralised P1 for the angry face, confirming that the N2pc was not driven by lower-level properties, and suggesting that it was instead driven by the threat value of the angry faces. Unexpectedly, the lateralised P1 was modulated by training. The contralateral P1 was greater to angry faces during angry training and greater to neutral faces during neutral training. Because the same face cues were presented across each block of the experiment, this modulation of the P1 was not driven by lower-level properties. The lateralised P1 is thought to reflect a 'gain control mechanism' that enhances visual processing of salient stimuli (Hillyard \& Anllo-Vento, 1998). Thus, the findings here suggest that participants selectively enhanced and processed the training-congruent faces during training. While this finding was unexpected, it is not inconsistent with other literature on the lateralised P1. Studies have shown that the task can modulate the lateralised P1; for example, Hickey and colleagues (2006) found that the lateralised P1 was affected by the reward value of a stimulus. However, since the goal of Experiment 2 was to investigate the effects of training on the N2pc, not the lateralised P1, further research is needed to follow up this finding. Taken together, these findings suggest that the null effect of training on the N2pc was not a product of experiment design. Instead, the results here suggest that participants engaged their attention with the angry face regardless of the training contingency.

Although there was no effect of training on the N2pc, participants' behaviour was sensitive to the training contingency. Participants moved their eyes toward the training-congruent faces during training. This dissociation between behaviour and the N2pc suggests that disengagement from threat, rather than engagement with threat, underlies ABM's effect on behaviour. Notably, it was not necessarily disadvantageous to engage with the angry face during neutral training. Although the probe always appeared in the location of the neutral face during neutral training, the angry face was still task-relevant, as the probe would always appear in the location opposite the angry face. Thus, provided that participants had sufficient attentional control to disengage from the angry face and move their attention to the predicted location of the probe, the angry face could still serve as an informative cue of the next location with which participants should engage their attention.

Other studies of the attentional mechanisms involved in ABM have also suggested that disengagement but not engagement is involved in ABM. For example, ABM affects late 
but not early ERP components (Eldar \& Bar-Haim, 2010; Suway et al., 2013; but see O’Toole \& Dennis, 2012), and biases at long but not short SOAs (Koster et al., 2010). ABM with subliminally-presented cues does not affect the threat bias (Maoz et al., 2013), suggesting that changes in bias require conscious awareness of the cues, as would be expected for disengagement. In addition, both threat and neutral training reduce anxiety (Klumpp \& Amir, 2009), suggesting that the clinical benefits of training arise through improved attentional control generally, and not selective attention away from threat. All of these studies suggest that disengagement from threat, not engagement with threat, is affected by ABM.

Converging evidence also comes from a very recent brain stimulation study (Clarke, Browning, Hammond, Notebaert, \& MacLeod, in press). Participants who received transcranial direct current stimulation to the lateral pre-frontal cortex (IPFC) showed a training-congruent bias after ABM, while participants in a sham stimulation condition showed no such change in bias. The IPFC is involved in attentional control (Corbetta \& Shulman, 2002), so these findings suggest that ABM may achieve its affects through improving attentional control and thus the ability to disengage attention from threat. This study is the first to show a causal relationship between PFC activity and the efficacy of ABM.

The null effect of training on the N2pc is inconsistent with studies of spatial predictability in the non-emotional attention literature. In a non-threat contextual cuing task, participants showed a greater N2pc for a target appearing in a predictable than an unpredictable location (Schankin, 2010), and the greater the effect of this predictability on RTs, the greater the N2pc effect (Schankin, 2009). Why was the N2pc modulated by predictability in these studies but not in the current study? It is possible that the threat N2pc arises from different neural sources than the non-threat N2pc. Indeed, some researchers have proposed that attention to threat operates through a different pathway from other attentional processes, and studies have found additive effects of attention to threat and other attentional processes (see Pourtois, Schettino, \& Vuilleumier, 2013, for a review). This hypothesis is also consistent with the fear module theory (Öhman \& Mineka, 2001), which proposes that the fear module evolved before cognition, and as such it is resistant to modification by 'higher level' cognition (i.e., attentional control). It follows that the threat N2pc may be less malleable than the non-threat N2pc. Indeed, the N2pc for threatening faces is unaffected by task or perceptual load (Foster, 2013; Ikeda et al., 2013; Shaw et al., 2011). If the threat N2pc reflects engagement that is driven by the fear module, then it is unlikely to be modified by a cognitive task like ABM. 
Future studies could explore the difference between the threat and non-threat N2pc with an ABM task. The effect of predictability on the non-threat N2pc could be tested with neutral cues (e.g., two letters, with training conditions in which the probe always appears in the location of one of the letters). If the non-threat $\mathrm{N} 2 \mathrm{pc}$ is affected by the predictability of the probe, participants would acquire an N2pc for the training-congruent letter during training. The effect of predictability on the threat N2pc could be tested with the same task, except one of the letters would be aversively conditioned. Participants would be expected to show an N2pc for the aversively-conditioned letter. If the threat N2pc is less malleable than the non-threat $\mathrm{N} 2 \mathrm{pc}$, this $\mathrm{N} 2 \mathrm{pc}$ for the aversively-conditioned letter would not be modulated by training.

\section{Future Directions}

Other ERP Components. A number of other ERP components may provide further insight into the mechanisms that underlie ABM. One such component is the $\mathrm{P} 1$ to the probe, which is greater to probes appearing in an attended (versus unattended) location. Dot-probe studies of the probe P1 find that it is greater when the probe appears in the location of the threat face as opposed to the neutral face (Brosch, Sander, Pourtois, \& Scherer, 2008; Pourtois et al., 2004). This P1 to the threat probe has an additive effect with the non-threat $\mathrm{N} 2 \mathrm{pc}$ for a bright attention-capturing flash, suggesting that the probe P1 and N2pc index separate attentional processes related to threat processing (Brosch et al., 2008). These findings suggest that threat affects the later perceptual enhancement of the probe appearing in the same spatial location as the threat face. No ERP studies of ABM (Eldar \& Bar-Haim, 2010; O’Toole \& Dennis, 2012; Suway et al., 2013) have analysed the probe P1.

In the present study, some design constraints prevented me from analysing the probe P1. Specifically, because my goal was to simulate the ABM task as it occurs in the clinical literature, I maintained a constant SOA of 500ms. However, to accurately measure the probe $\mathrm{P} 1$ it is necessary to jitter the onset of the probe so that participants cannot anticipate it. Without jittering, a large component (called the contingent negative variation, CNV; Tecce, 1972) appears before the onset of the probe, making it difficult to detect the P1. A second constraint is that analysis of the probe P1 entails comparing its magnitude when the probe appears in the location of the angry versus the neutral faces. It is therefore not possible to analyse the probe $\mathrm{P} 1$ in a $100 \%$ predictive training block.

However, in future studies a slightly modified design could be used to measure both the N2pc to the faces and the probe P1. If ABM affects the perceptual enhancement of the training-congruent probe, training will increase the P1 to the training-congruent probe. If, as I 
propose, participants in neutral training initially engage their attention with the angry face but then disengage to shift their attention to the neutral probe, they would show both a P1 to the neutral probe and an N2pc for the angry face. Such a pattern of results would provide strong support for the hypothesis that training affects attentional disengagement, but not engagement with threat.

Another component that could provide further insight into the mechanisms involved in ABM is the sustained posterior contralateral negativity (SPCN). The SPCN has the same characteristics as the $\mathrm{N} 2 \mathrm{pc}$ except it appears in a later time window (350 to 500ms). The SPCN is thought to index visual short-term memory, and indicates sustained attention to a contralateral stimulus (Jolicoeur, Brisson, \& Robitaille, 2008). To date, no studies have investigated the effects of ABM on the SPCN. However, participants show an SPCN for threat faces (Feldmann-Wüstefeld et al., 2011), indicating that they maintain engagement with threat over time. In an ABM task, if participants in the angry condition maintained engagement with the angry faces, the SPCN for the angry faces would increase during angry training. If participants in the neutral condition initially engaged but then disengaged from the angry face, the SPCN for the angry face would decrease (or reverse) during neutral training. However, the SPCN cannot be assessed during a typical ABM task because it appears later than the N2pc, thus is more easily contaminated by eye movements and the CNV occurring before the probe. As with the P1 to the probe, modification of the ABM procedure would make the task less consistent with more clinically-oriented studies of ABM, but might provide important insights into the mechanisms of bias modification.

Anxiety. Only non-anxious individuals were included in the current experiments because I was investigating the effects of ABM on attentional engagement, which is presumably unimpaired in healthy individuals. However, the ABM literature has typically focused on the clinical effects of ABM in anxious populations. Anxious individuals have a greater threat bias than non-anxious individuals, and ABM reduces anxiety and the threat bias in anxious participants (Beard et al., 2012; Cisler \& Koster, 2010; Hakamata et al., 2010). Attentional control theory (Eysenck et al., 2007; Eysenck \& Derakshan, 2011) proposes that anxiety impairs attentional control, which leads to increased attention to threat. This impairment in attentional control is thought to arise because anxious individuals distribute their attention broadly so that they can detect threat more readily in the environment. Thus, they do not focus their attention on only one stimulus in the environment unless it is threatening. ABM may improve attentional control by making it advantageous to attend to only one stimulus (the predictive cue). As a result, $\mathrm{ABM}$ may reduce anxiety by improving 
attentional control, thereby improving participants' ability to direct their attention away from task-irrelevant threat.

Anxiety-related deficits in attentional control may also explain why ABM affects the threat bias in anxious but not non-anxious individuals. Anxious individuals may show changes in bias after $\mathrm{ABM}$ because it trains their attentional control and thus improves their ability to disengage from the threat face at the post-training assessment when the faces are no longer predictive of the probe's location. In contrast, non-anxious individuals already have good attentional control. They therefore respond well to training and acquire a bias during training when it is advantageous to do so, but easily revert to unbiased attention during the post-training assessment. In the current experiment, behavioural effects were observed after only 96 training trials. However, if ABM achieves its effects by training attentional control, these behavioural effects may take longer to arise in anxious individuals.

There may be other differences in the effects of ABM in anxious and non-anxious individuals. Anxious participants show a greater N2pc for angry faces relative to non-anxious participants (Fox et al., 2008), suggesting that anxious individuals have increased engagement with threat. However, the current experiments showed that the N2pc for angry faces is not modulated by training in non-anxious individuals. It would be interesting to investigate whether the $\mathrm{N} 2 \mathrm{pc}$ for the angry faces is modulated by ABM in anxious individuals. The N2pc for angry faces may be more malleable in anxious individuals and therefore change during training. Alternatively, the N2pc in anxious individuals may not change during training, consistent with the current findings. The latter finding would suggest that the N2pc for threat may reflect a risk factor for anxiety, or that the N2pc may take longer to develop over time as an individual develops anxiety.

\section{Limitations}

In all of the current experiments, participants were instructed to maintain fixation throughout the ABM task. The ABM task also encouraged this fixation by requiring participants to make a discrimination judgement that involved comparing the probe and the fixation cross. Despite these efforts, HEOG analyses showed that participants made systematic eye movements in the training-congruent direction during training in Experiment 2. A large number of participants were then excluded from the N2pc analyses because their large eye movements could confound analyses. This high exclusion rate due to eye movements may explain why the previous three ERP studies of ABM did not investigate changes in ERPs during training. Modifying the ABM task to make probe discrimination easier may reduce participants' eye movements toward the faces, but Experiment 1a shows 
that the training-congruent bias is short-lived if the probe is easy to discriminate. It is also possible that a training-congruent bias was observed in Experiment $1 \mathrm{~b}$ because participants had to move their eyes to discriminate the probe. To the best of my knowledge, no study has used eye tracking during $\mathrm{ABM}$ to test the effects of training on eye movements. It is possible that these systematic eye movements are unavoidable as they are necessary for ABM's effects. Early components (e.g., lateralised P1 to the faces) that occur before eye movements can be programmed (Lambert, Norris, Naikar, \& Aitken, 2000) may be the only components that can be studied without being contaminated by eye movements.

\section{Conclusions}

The goal of this thesis was to extend our understanding of the mechanisms involved in ABM. In Experiment 1a and 1b, I developed an ABM task that trained a bias toward either angry or neutral faces in non-anxious individuals during training. The findings of Experiment 1 suggest that the failure of other studies to show training effects in non-anxious individuals is related to the way that the bias is tested only before and after training. In addition, Experiment 1 shows that the attentional demands of the ABM task are important for training and assessing the threat bias.

In Experiment 2, I then used this ABM task to investigate changes in the N2pc (an index of attentional engagement) before, during, and after training. Experiment 2 found that there was no effect of training on post-training bias scores. However, participants moved their eyes in the training-congruent direction during training, suggesting that they had acquired a training-congruent bias. An overall N2pc for the angry faces were observed, consistent with previous literature (Foster, 2013; Grimshaw et al., 2014; Holmes et al., 2009, in press). However, the N2pc was not affected by training. This observation suggests that $\mathrm{ABM}$ does not affect engagement with threat, and supports a growing literature that suggests that ABM improves an individual's attentional control, which leads to an increased ability to disengage from threat.

Analysis of the lateralised P1 to the faces confirmed that the N2pc was not driven by lower-level differences between the angry and the neutral faces. Unexpectedly, the P1 was affected by training, and was greatest in the training-congruent direction during training. This finding suggests that participants selectively enhanced the training-congruent face during training. However, this finding was not predicted by the original hypotheses, so further research is needed to confirm and explore this effect.

The research in the current experiments is important because it provides insight into the mechanisms that underlie the threat bias and its modification. The results in the present 
study suggest that training paradigms that target attentional control and disengagement rather than engagement may be more effective at reducing anxiety. With this information, ABM tasks could be modified to include faces presented for longer durations, thus tapping into later disengagement mechanisms, or to use non-threat cues, since the presence of threat may not be necessary for training disengagement. In addition to the theoretical implications, understanding the mechanisms underlying the threat bias and its modification is important for developing effective and targeted treatments for anxiety. 


\section{References}

American Electroencephalographic Society. (1994). Guidelines for standard electrode position nomenclature. Journal of Clinical Neurophysiology, 11(1), 111-113. Retrieved from http://journals.lww.com/clinicalneurophys/pages/default.aspx

Bar-Haim, Y. (2010). Research review: Attention bias modification (ABM): A novel treatment for anxiety disorders. Journal of Child Psychology and Psychiatry, and Allied Disciplines, 51(8), 859-870. doi:10.1111/j.1469-7610.2010.02251.x

Bar-Haim, Y., Lamy, D., Pergamin, L., Bakermans-Kranenburg, M. J., \& van IJzendoorn, M. H. (2007). Threat-related attentional bias in anxious and nonanxious individuals: A meta-analytic study. Psychological Bulletin, 133(1), 1-24. doi:10.1037/00332909.133.1.1

Beard, C. (2011). Cognitive bias modification for anxiety: Current evidence and future directions. Expert Review of Neurotherapeutics, 11(2), 299-311. doi:10.1586/ern.10.194

Beard, C., Sawyer, A. T., \& Hofmann, S. G. (2012). Efficacy of attention bias modification using threat and appetitive stimuli: A meta-analytic review. Behavior Therapy, 43(4), 724-740. doi:10.1016/j.beth.2012.01.002

Beck, D. M., \& Kastner, S. (2009). Top-down and bottom-up mechanisms in biasing competition in the human brain. Vision Research, 49(10), 1154-1165. doi:10.1016/j.visres.2008.07.012

Bekker, M. H. J., \& van Mens-Verhulst, J. (2007). Anxiety disorders: Sex differences in prevalence, degree, and background, but gender-neutral treatment. Gender Medicine, 4 Suppl B, S178-193. doi:10.1016/S1550-8579(07)80057-X

Belopolsky, A. V, Zwaan, L., Theeuwes, J., \& Kramer, A. F. (2007). The size of an attentional window modulates attentional capture by color singletons. Psychonomic Bulletin \& Review, 14(5), 934-938. doi:10.3758/BF03194124

Bishop, S. J. (2007). Neurocognitive mechanisms of anxiety: An integrative account. Trends in Cognitive Sciences, 11(7), 307-316. doi:10.1016/j.tics.2007.05.008

Brosch, T., Pourtois, G., Sander, D., \& Vuilleumier, P. (2011). Additive effects of emotional, endogenous, and exogenous attention: Behavioral and electrophysiological evidence. Neuropsychologia, 49(7), 1779-1787. doi:10.1016/j.neuropsychologia.2011.02.056

Brosch, T., Sander, D., Pourtois, G., \& Scherer, K. R. (2008). Beyond fear: Rapid spatial orienting toward positive emotional stimuli. Psychological Science, 19(4), 362-370. doi:10.1111/j.1467-9280.2008.02094.x 
Browning, M., Holmes, E. A., \& Murphy, S. (2010). Lateral prefrontal cortex mediates the cognitive modification of attentional bias. Biological Psychiatry, 67(10), 919-925. doi:10.1016/j.biopsych.2009.10.031

Buodo, G., Sarlo, M., \& Munafò, M. (2010). The neural correlates of attentional bias in blood phobia as revealed by the N2pc. Social Cognitive and Affective Neuroscience, 5(1), 2938. doi:10.1093/scan/nsp050

Cisler, J. M., Bacon, A. K., \& Williams, N. L. (2007). Phenomenological characteristics of attentional biases towards threat: A critical review. Cognitive Therapy and Research, 33(2), 221-234. doi:10.1007/s10608-007-9161-y

Cisler, J. M., \& Koster, E. H. W. (2010). Mechanisms of attentional biases towards threat in anxiety disorders: An integrative review. Clinical Psychology Review, 30(2), 203-216. doi:10.1016/j.cpr.2009.11.003

Cisler, J. M., Ries, B. J., \& Widner, R. L. (2007). Examining information processing biases in spider phobia using the rapid serial visual presentation paradigm. Journal of Anxiety Disorders, 21(8), 977-990. doi:10.1016/j.janxdis.2006.10.011

Clark, L. A, \& Watson, D. (1991). Tripartite model of anxiety and depression: psychometric evidence and taxonomic implications. Journal of Abnormal Psychology, 100(3), 316336. doi:10.1037/0021-843X.100.3.316

Clarke, P. J. F., Browning, M., Hammond, G., Notebaert, L., \& MacLeod, C. (in press). The causal role of the dorsolateral prefrontal cortex in the modification of attentional bias: Evidence from transcranial direct current stimulation. Biological Psychiatry. doi:10.1016/j.biopsych.2014.03.003

Clarke, P. J. F., Macleod, C., \& Guastella, A. J. (2013). Assessing the role of spatial engagement and disengagement of attention in anxiety-linked attentional bias: A critique of current paradigms and suggestions for future research directions. Anxiety, Stress, and Coping, 26(1), 1-19. doi:10.1080/10615806.2011.638054

Cooper, R. M., \& Langton, S. R. H. (2006). Attentional bias to angry faces using the dotprobe task? It depends when you look for it. Behaviour Research and Therapy, 44(9), 1321-1329. doi:10.1016/j.brat.2005.10.004

Corbetta, M., \& Shulman, G. L. (2002). Control of goal-directed and stimulus-driven attention in the brain. Nature Reviews. Neuroscience, 3(3), 201-215. doi:10.1038/nrn755 
Derryberry, D., \& Reed, M. a. (2002). Anxiety-related attentional biases and their regulation by attentional control. Journal of Abnormal Psychology, 111(2), 225-236. doi:10.1037//0021-843X.111.2.225

Desimone, R., \& Duncan, J. (1995). Neural mechanisms of selective visual attention. Annual Review of Neuroscience, 18, 193-222. doi:10.1146/annurev.ne.18.030195.001205

Dunlap, W. P., Cortina, J. M., Vaslow, J. B., \& Burke, M. J. (1996). Meta-analysis of experiments with matched groups or repeated measures designs. Psychological Methods, 1(2), 170-177. doi:10.1037//1082-989X.1.2.170

Eastwood, J., Smile, D., Oakman, J., Farvolden, P., van Ameringen, M., Mancini, C., \& Merikle, P. (2005). Individuals with social phobia are biased to become aware of negative faces. Visual Cognition, 12(1), 159-179. doi:10.1080/13506280444000175

Eimer, M., \& Kiss, M. (2007). Attentional capture by task-irrelevant fearful faces is revealed by the N2pc component. Biological Psychology, 74(1), 108-112. doi:10.1016/j.biopsycho.2006.06.008

Eldar, S., \& Bar-Haim, Y. (2010). Neural plasticity in response to attention training in anxiety. Psychological Medicine, 40(4), 667-77. doi:10.1017/S0033291709990766

Eldar, S., Ricon, T., \& Bar-Haim, Y. (2008). Plasticity in attention: Implications for stress response in children. Behaviour Research and Therapy, 46(4), 450-461. doi:10.1016/j.brat.2008.01.012

Eldar, S., Yankelevitch, R., Lamy, D., \& Bar-Haim, Y. (2010). Enhanced neural reactivity and selective attention to threat in anxiety. Biological Psychology, 85(2), 252-257. doi:10.1016/j.biopsycho.2010.07.010

Everaert, T., Spruyt, A., \& De Houwer, J. (2013). On the malleability of automatic attentional biases: Effects of feature-specific attention allocation. Cognition and Emotion, 27(3), 37-41. doi:10.1080/02699931.2012.712949

Eysenck, M. W., \& Derakshan, N. (2011). New perspectives in attentional control theory. Personality and Individual Differences, 50(7), 955-960. doi:10.1016/j.paid.2010.08.019

Eysenck, M. W., Derakshan, N., Santos, R., \& Calvo, M. G. (2007). Anxiety and cognitive performance: Attentional control theory. Emotion, 7(2), 336-353. doi:10.1037/15283542.7.2.336

Fajkowska, M., \& Derryberry, D. (2010). Psychometric properties of Attentional Control Scale: The preliminary study on a Polish sample. Polish Psychological Bulletin, 41(1), 1-7. doi:10.2478/s10059-010-0001-7 
Fecica, A. M., \& Stolz, J. a. (2008). Facial affect and temporal order judgments. Experimental Psychology, 55(1), 3-8. doi:10.1027/1618-3169.55.1.3

Feldmann-Wüstefeld, T., Schmidt-Daffy, M., \& Schubö, A. (2011). Neural evidence for the threat detection advantage: Differential attention allocation to angry and happy faces. Psychophysiology, 48(5), 697-707. doi:10.1111/j.1469-8986.2010.01130.x

Foster, J. J. (2013). Attention capture by angry faces depends on the distribution of attention. Victoria University of Wellington. Retrieved from researcharchive.vuw.ac.nz/xmlui/handle/10063/2749

Fox, E., Derakshan, N., \& Shoker, L. (2008). Trait anxiety modulates the electrophysiological indices of rapid spatial orienting towards angry faces. Neuroreport, 19(3), 259-263. doi:10.1097/WNR.0b013e3282f53d2a

Fox, E., Russo, R., \& A., G. G. (2005). Anxiety modulates the degree of attentive resources required to process emotional faces. Cognitive, Affective, \& Behavioral Neuroscience, 5(4), 396-404. doi:10.3758/CABN.5.4.396

Fox, E., Russo, R., Bowles, R., \& Dutton, K. (2001). Do threatening stimuli draw or hold visual attention in subclinical anxiety? Journal of Experimental Psychology: General, 130(4), 681-700. doi:10.1037//0096-3445.130.4.681

Frewen, P. a, Dozois, D. J. a, Joanisse, M. F., \& Neufeld, R. W. J. (2008). Selective attention to threat versus reward: Meta-analysis and neural-network modeling of the dot-probe task. Clinical Psychology Review, 28(2), 307-337. doi:10.1016/j.cpr.2007.05.006

Frischen, A., Eastwood, J. D., \& Smilek, D. (2008). Visual search for faces with emotional expressions. Psychological Bulletin, 134(5), 662-676. doi:10.1037/0033-2909.134.5.662

Greenhouse, S. W., \& Geisser, S. (1959). On methods in the analysis of profile data. Psychometrika, 24(2), 95-112. doi:10.1007/BF02289823

Grimshaw, G. M., Foster, J. J., \& Corballis, P. M. (2014). Frontal and parietal EEG asymmetries interact to predict attentional bias to threat. Manuscript submitted for publication.

Hakamata, Y., Lissek, S., Bar-Haim, Y., Britton, J. C., Fox, N. A., Leibenluft, E., ... Pine, D. S. (2010). Attention bias modification treatment: A meta-analysis toward the establishment of novel treatment for anxiety. Biological Psychiatry, 68(11), 982-990. doi:10.1016/j.biopsych.2010.07.021

Hallion, L. S., \& Ruscio, A. M. (2011). A meta-analysis of the effect of cognitive bias modification on anxiety and depression. Psychological Bulletin, 137(6), 940-958. doi:10.1037/a0024355 
Heeren, A., Lievens, L., \& Philippot, P. (2011). How does attention training work in social phobia: Disengagement from threat or re-engagement to non-threat? Journal of Anxiety Disorders, 25(8), 1108-1115. doi:10.1016/j.janxdis.2011.08.001

Heeren, A., Reese, H. E., McNally, R. J., \& Philippot, P. (2012). Attention training toward and away from threat in social phobia: Effects on subjective, behavioral, and physiological measures of anxiety. Behaviour Research and Therapy, 50(1), 30-39. doi:10.1016/j.brat.2011.10.005

Hickey, C., McDonald, J. J., \& Theeuwes, J. (2006). Electrophysiological evidence of the capture of visual attention. Journal of Cognitive Neuroscience, 18(4), 604-613. doi:10.1162/jocn.2006.18.4.604

Hillyard, S. a, \& Anllo-Vento, L. (1998). Event-related brain potentials in the study of visual selective attention. Proceedings of the National Academy of Sciences, 95(3), 781-787. doi:10.1073/pnas.95.3.781

Hillyard, S. a, Vogel, E. K., \& Luck, S. J. (1998). Sensory gain control (amplification) as a mechanism of selective attention: Electrophysiological and neuroimaging evidence. Philosophical Transactions of the Royal Society of London. Series B, Biological Sciences, 353(1373), 1257-1270. doi:10.1098/rstb.1998.0281

Holmes, A., Bradley, B. P., Kragh Nielsen, M., \& Mogg, K. (2009). Attentional selectivity for emotional faces: Evidence from human electrophysiology. Psychophysiology, 46(1), 62-68. doi:10.1111/j.1469-8986.2008.00750.x

Holmes, A., Mogg, K., de Fockert, J., Nielsen, M. K., \& Bradley, B. P. (in press). Electrophysiological evidence for greater attention to threat when cognitive control resources are depleted. Cognitive, Affective \& Behavioral Neuroscience. doi:10.3758/s13415-013-0212-4

Ikeda, K., Sugiura, A., \& Hasegawa, T. (2013). Fearful faces grab attention in the absence of late affective cortical responses. Psychophysiology, 50(1), 60-69. doi:10.1111/j.14698986.2012.01478.x

Jolicoeur, P., Brisson, B., \& Robitaille, N. (2008). Dissociation of the N2pc and sustained posterior contralateral negativity in a choice response task. Brain Research, 1215, 160172. doi:10.1016/j.brainres.2008.03.059

Klumpp, H., \& Amir, N. (2009). Preliminary study of attention training to threat and neutral faces on anxious reactivity to a social stressor in social anxiety. Cognitive Therapy and Research, 34(3), 263-271. doi:10.1007/s10608-009-9251-0 
Koster, E. H. W., Baert, S., Bockstaele, M., \& De Raedt, R. (2010). Attentional retraining procedures: Manipulating early or late components of attentional bias? Emotion, 10(2), 230-236. doi:10.1037/a0018424

Koster, E. H. W., Crombez, G., Van Damme, S., Verschuere, B., \& De Houwer, J. (2005). Signals for threat modulate attentional capture and holding: Fear-conditioning and extinction during the exogenous cueing task. Cognition \& Emotion, 19(5), 771-780. doi:10.1080/02699930441000418

Koster, E. H. W., Leyman, L., Raedt, R. De, \& Crombez, G. (2006). Cueing of visual attention by emotional facial expressions: The influence of individual differences in anxiety and depression. Personality and Individual Differences, 41(2), 329-339. doi:10.1016/j.paid.2005.12.022

Koster, E. H. W., Verschuere, B., Crombez, G., \& Van Damme, S. (2005). Time-course of attention for threatening pictures in high and low trait anxiety. Behaviour Research and Therapy, 43(8), 1087-1098. doi:10.1016/j.brat.2004.08.004

Lambert, A., Naikar, N., McLachlan, K., \& Aitken, V. (1999). A new component of visual orienting: Implicit effects of peripheral information and subthreshold cues on covert attention. Journal of Experimental Psychology: Human Perception and Performance, 25(2), 321-340. doi:10.1037//0096-1523.25.2.321

Lambert, A., Norris, A., Naikar, N., \& Aitken, V. (2000). Effects of informative peripheral cues on eye movements: Revisiting William James' “derived attention”. Visual Cognition, 7(5), 545-569. doi:10.1080/135062800407194

Li, W., Zinbarg, R. E., \& Paller, K. A. (2007). Trait anxiety modulates supraliminal and subliminal threat: Brain potential evidence for early and late processing influences. Cognitive, Affective \& Behavioral Neuroscience, 7(1), 25-36. doi:10.3758/CABN.7.1.25

Lichtenstein-Vidne, L., Henik, A., \& Safadi, Z. (2012). Task relevance modulates processing of distracting emotional stimuli. Cognition \& Emotion, 26(1), 42-52. doi:10.1080/02699931.2011.567055

Lien, M.-C., Taylor, R., \& Ruthruff, E. (2013). Capture by fear revisited: An electrophysiological investigation. Journal of Cognitive Psychology, 25(7), 873-888. doi:10.1080/20445911.2013.833933

Lins, O. G., Picton, T. W., Berg, P., \& Scherg, M. (1993a). Ocular artifacts in EEG and event-related potentials. I: Scalp topography. Brain Topography, 6(1), 51-63. doi:10.1007/BF01234127 
Lins, O. G., Picton, T. W., Berg, P., \& Scherg, M. (1993b). Ocular artifacts in recording EEGs and event-related potentials. II: Source dipoles and source components. Brain Topography, 6(1), 65-78. doi:10.1007/BF01234128

Lipp, O. V, \& Derakshan, N. (2005). Attentional bias to pictures of fear-relevant animals in a dot probe task. Emotion, 5(3), 365-369. doi:10.1037/1528-3542.5.3.365

Lovibond, P. F., \& Lovibond, S. H. (1995). The structure of negative emotional states: Comparison of the Depression Anxiety Stress Scales (DASS) with the Beck Depression and Anxiety Inventories. Behaviour Research and Therapy, 33(3), 335-343. doi:10.1016/0005-7967(94)00075-U

Luck, S. J. (2012). Electrophysiological correlates of the focusing of attention within complex visual scenes: N2pc and related ERP components. In S. J. Luck \& E. S. Kappenman (Eds.), The Oxford Handbook of Event-Related Potential Components (pp. 329-360). New York: Oxford University Press. doi:10.1093/oxfordhb/9780195374148.013.0161

Luck, S. J., \& Hillyard, S. A. (1994). Spatial filtering during visual search: Evidence from human electrophysiology. Journal of Experimental Psychology: Human Perception and Performance, 20(5), 1000-1014. doi:10.1037/0096-1523.20.5.1000

Luck, S. J., Woodman, G., \& Vogel, E. (2000). Event-related potential studies of attention. Trends in Cognitive Sciences, 4(11), 432-440. doi:10.1016/S1364-6613(00)01545-X

MacLeod, C., Mathews, A., \& Tata, P. (1986). Attentional bias in emotional disorders. Journal of Abnormal Psychology, 95(1), 15-20. doi:10.1037/0021-843X.95.1.15

MacLeod, C., Rutherford, E., Campbell, L., Ebsworthy, G., \& Holker, L. (2002). Selective attention and emotional vulnerability: Assessing the causal basis of their association through the experimental manipulation of attentional bias. Journal of Abnormal Psychology, 111(1), 107-123. doi:10.1037/0021-843X.111.1.107

Mallan, K. M., Lipp, O. V, \& Cochrane, B. (2013). Slithering snakes, angry men and outgroup members: What and whom are we evolved to fear? Cognition \& Emotion, 27(7), 1168-1180. doi:10.1080/02699931.2013.778195

Maoz, K., Abend, R., Fox, N. A., Pine, D. S., \& Bar-Haim, Y. (2013). Subliminal attention bias modification training in socially anxious individuals. Frontiers in Human Neuroscience, 7, 1-12. doi:10.3389/fnhum.2013.00389

Massar, S. a a, Mol, N. M., Kenemans, J. L., \& Baas, J. M. P. (2011). Attentional bias in high- and low-anxious individuals: Evidence for threat-induced effects on engagement 
and disengagement. Cognition \& Emotion, 25(5), 805-817.

doi:10.1080/02699931.2010.515065

Mathews, A., \& Mackintosh, B. (1998). A Cognitive Model of Selective Processing in Anxiety. Cognitive Therapy and Research, 22(6), 539-560.

doi:10.1023/A:1018738019346

Mathews, A., \& MacLeod, C. (2002). Induced processing biases have causal effects on anxiety. Cognition \& Emotion, 16(3), 331-354. doi:10.1080/02699930143000518

Mauchly, J. (1940). Significance test for sphericity of a normal $n$-variate distribution. The Annals of Mathematical Statistics, 11(2), 204-209. doi:10.1214/aoms/1177731915

Mazza, V., Turatto, M., \& Caramazza, A. (2009). Attention selection, distractor suppression and N2pc. Cortex, 45(7), 879-890. doi:10.1016/j.cortex.2008.10.009

McHugo, M., Olatunji, B. O., \& Zald, D. H. (2013). The emotional attentional blink: What we know so far. Frontiers in Human Neuroscience, 7, 1-9. doi:10.3389/fnhum.2013.00151

Meyer, T. J., Miller, M. L., Metzger, R. L., \& Borkovec, T. D. (1990). Development and validation of the Penn State worry questionnaire. Behaviour Research and Therapy, 28(6), 487-495. doi:10.1016/0005-7967(90)90135-6

Mogg, K., \& Bradley, B. P. (2002). Selective orienting of attention to masked threat faces in social anxiety. Behaviour Research and Therapy, 40(12), 1403-14. doi:10.1016/S00057967(02)00017-7

Mogg, K., \& Bradley, B. P. (2005). Attentional bias in generalized anxiety disorder versus depressive disorder. Cognitive Therapy and Research, 29(1), 29-45. doi:10.1007/s10608-005-1646-y

Mogg, K., Bradley, B. P., de Bono, J., \& Painter, M. (1997). Time course of attentional bias for threat information in non-clinical anxiety. Behaviour Research and Therapy, 35(4), 297-303. doi:10.1016/S0005-7967(96)00109-X

Mogg, K., Bradley, B. P., Miles, F., \& Dixon, R. (2004). Time course of attentional bias for threat scenes: Testing the vigilance avoidance hypothesis. Cognition \& Emotion, 18(5), 689-700. doi:10.1080/02699930341000158

Mogg, K., Philippot, P., \& Bradley, B. P. (2004). Selective attention to angry faces in clinical social phobia. Journal of Abnormal Psychology, 113(1), 160-165. doi:10.1037/0021843X.113.1.160 
Notebaert, L., Crombez, G., Van Damme, S., De Houwer, J., \& Theeuwes, J. (2011). Signals of threat do not capture, but prioritize, attention: A conditioning approach. Emotion, 11(1), 81-89. doi:10.1037/a0021286

O’Toole, L., \& Dennis, T. A. (2012). Attention training and the threat bias: An ERP study. Brain and Cognition, 78(1), 63-73. doi:10.1016/j.bandc.2011.10.007

Öhman, A., \& Mineka, S. (2001). Fears, phobias, and preparedness: Toward an evolved module of fear and fear learning. Psychological Review, 108(3), 483-522. doi:10.1037//0033-295X.108.3.483

Ouimet, A. J., Gawronski, B., \& Dozois, D. J. A. (2009). Cognitive vulnerability to anxiety: A review and an integrative model. Clinical Psychology Review, 29(6), 459-470. doi:10.1016/j.cpr.2009.05.004

Perron, R., Lefebvre, C., Robitaille, N., Brisson, B., Gosselin, F., Arguin, M., \& Jolicoeur, P. (2009). Attentional and anatomical considerations for the representation of simple stimuli in visual short-term memory: Evidence from human electrophysiology. Psychological Research, 73(2), 222-232. doi:10.1007/s00426-008-0214-y

Piccinelli, M., \& Wilkinson, G. (2000). Gender differences in depression: Critical review. The British Journal of Psychiatry, 177(6), 486-492. doi:10.1192/bjp.177.6.486

Posner, M. I. (1980). Orienting of attention. Quarterly Journal of Experimental Psychology, 32(1), 3-25. doi:10.1080/00335558008248231

Pourtois, G., Grandjean, D., Sander, D., \& Vuilleumier, P. (2004). Electrophysiological correlates of rapid spatial orienting towards fearful faces. Cerebral Cortex, 14(6), 619633. doi:10.1093/cercor/bhh023

Pourtois, G., Schettino, A., \& Vuilleumier, P. (2013). Brain mechanisms for emotional influences on perception and attention: What is magic and what is not. Biological Psychology, 92(3), 492-512. doi:10.1016/j.biopsycho.2012.02.007

Purkis, H. M., \& Lipp, O. V. (2007). Automatic attention does not equal automatic fear: Preferential attention without implicit valence. Emotion, 7(2), 314-323. doi:10.1037/1528-3542.7.2.314

Rinck, M., Becker, E. S., Kellermann, J., \& Roth, W. T. (2003). Selective attention in anxiety: Distraction and enhancement in visual search. Depression and Anxiety, 18(1), 18-28. doi:10.1002/da.10105

Rossignol, M., Philippot, P., Bissot, C., Rigoulot, S., \& Campanella, S. (2012). Electrophysiological correlates of enhanced perceptual processes and attentional capture 
by emotional faces in social anxiety. Brain Research, 1460, 50-62. doi:10.1016/j.brainres.2012.04.034

Salemink, E., van den Hout, M. a, \& Kindt, M. (2007). Selective attention and threat: Quick orienting versus slow disengagement and two versions of the dot probe task. Behaviour Research and Therapy, 45(3), 607-615. doi:10.1016/j.brat.2006.04.004

Santesso, D., Meuret, A., \& Hofmann, S. G. (2008). Electrophysiological correlates of spatial orienting towards angry faces: A source localization study. Neuropsychologia, 46(5), 1338-1348. doi:10.1016/j.neuropsychologia.2007.12.013

Schettino, A., Loeys, T., \& Pourtois, G. (2013). No prior entry for threat-related faces: Evidence from temporal order judgments. PloS One, 8(4), 1-15. doi:10.1371/journal.pone.0062296

Schneider, W., Eschman, A., \& Zuccolotto, A. (2002a). E-prime reference guide. Pittsburgh, PA: Psychology Software Tools.

Schneider, W., Eschman, A., \& Zuccolotto, A. (2002b). E-prime user's guide. Pittsburgh, PA: Psychology Software Tools.

Shaw, K., Lien, M.-C., Ruthruff, E., \& Allen, P. a. (2011). Electrophysiological evidence of emotion perception without central attention. Journal of Cognitive Psychology, 23(6), 695-708. doi:10.1080/20445911.2011.586624

Springer, U. S., Rosas, A., McGetrick, J., \& Bowers, D. (2007). Differences in startle reactivity during the perception of angry and fearful faces. Emotion, 7(3), 516-525. doi:10.1037/1528-3542.7.3.516

Stolarova, M., Keil, A., \& Moratti, S. (2006). Modulation of the C1 visual event-related component by conditioned stimuli: Evidence for sensory plasticity in early affective perception. Cerebral Cortex, 16(6), 876-887. doi:10.1093/cercor/bhj031

Suway, J. G., White, L. K., Vanderwert, R. E., Bar-Haim, Y., Pine, D. S., \& Fox, N. A. (2013). Modification of threat-processing in non-anxious individuals: A preliminary behavioral and ERP study. Journal of Behavior Therapy and Experimental Psychiatry, 44(3), 285-292. doi:10.1016/j.jbtep.2012.11.006

Tecce, J. (1972). Contingent negative variation (CNV) and psychological processes in man. Psychological Bulletin, 77(2), 73-108. doi:10.1037/h0032177

Thayer, J. F., \& Johnsen, B. H. (2000). Sex differences in judgement of facial affect: A multivariate analysis of recognition errors. Scandinavian Journal of Psychology, 41(3), 243-246. doi:10.1111/1467-9450.00193 
Tipples, J., Young, A. W., Quinlan, P., Broks, P., \& Ellis, A. W. (2002). Searching for threat. The Quarterly Journal of Experimental Psychology. A, Human Experimental Psychology, 55(3), 1007-1026. doi:10.1080/02724980143000659

Tottenham, N., Tanaka, J. W., Leon, A. C., McCarry, T., Nurse, M., Hare, T. A., ... Nelson, C. (2009). The NimStim set of facial expressions: Judgments from untrained research participants. Psychiatry Research, 168(3), 242-249. doi:10.1016/j.psychres.2008.05.006

Trippe, R. H., Hewig, J., Heydel, C., Hecht, H., \& Miltner, W. H. R. (2007). Attentional Blink to emotional and threatening pictures in spider phobics: Electrophysiology and behavior. Brain Research, 1148(1987), 149-160. doi:10.1016/j.brainres.2007.02.035

Walter, W., Cooper, R., \& Aldridge, V. (1964). Contingent negative variation: An electric sign of sensori-motor association and expectancy in the human brain. Nature, 203, 380384. doi:10.1038/203380a0

West, G. L., Anderson, A. A. K., \& Pratt, J. (2009). Motivationally significant stimuli show visual prior entry: Evidence for attentional capture. Journal of Experimental Psychology. Human Perception and Performance, 35(4), 1032-1042. doi:10.1037/a0014493

Weymar, M., Gerdes, A. B. M., Löw, A., Alpers, G. W., \& Hamm, A. O. (2013). Specific fear modulates attentional selectivity during visual search: Electrophysiological insights from the N2pc. Psychophysiology, 50(2), 139-148. doi:10.1111/psyp.12008

Weymar, M., Keil, A., \& Hamm, A. O. (2013). Timing the fearful brain: Unspecific hypervigilance and spatial attention in early visual perception. Social Cognitive and Affective Neuroscience, 1-32. doi:10.1093/scan/nst044

Weymar, M., Löw, A., Ohman, A., \& Hamm, A. O. (2011). The face is more than its parts Brain dynamics of enhanced spatial attention to schematic threat. NeuroImage, 58(3), 946-954. doi:10.1016/j.neuroimage.2011.06.061

Williams, J. M. G., Watts, F. N., MacLeod, C., \& Mathews, A. (1988). Cognitive psychology and emotional disorders. John Wiley \& Sons.

Williams, M., Moss, S., Bradshaw, J., \& Mattingley, J. B. (2005). Look at me, I'm smiling: Visual search for threatening and nonthreatening facial expressions. Visual Cognition, 12(1), 29-50. doi:10.1080/13506280444000193

Yao, S., Ding, C., Qi, S., \& Yang, D. (in press). Value associations of emotional faces can modify the anger superiority effect: Behavioral and electrophysiological evidence. Social Cognitive and Affective Neuroscience. doi:10.1093/scan/nst056 
Yiend, J., \& Mathews, A. (2001). Anxiety and attention to threatening pictures. The Quarterly Journal of Experimental Psychology, 54A(3), 665-681. doi:10.1080/02724980042000462 